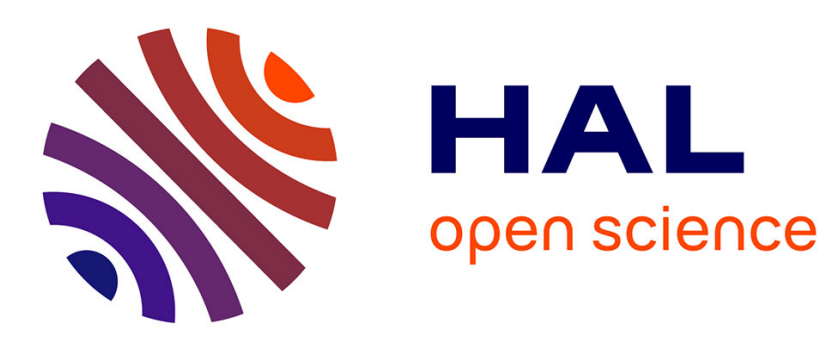

\title{
Transition entre les cultures néolithiques de Sesklo et de Dimini : les catégories céramiques
}

\author{
Jean-Paul Demoule, Kostas Gallis, Laurence Manolakakis
}

\section{To cite this version:}

Jean-Paul Demoule, Kostas Gallis, Laurence Manolakakis. Transition entre les cultures néolithiques de Sesklo et de Dimini : les catégories céramiques. Bulletin de Correspondance Hellenique, 1988, 112 (1), pp.1-58. 10.3406/bch.1988.1737 . hal-02551151

\section{HAL Id: hal-02551151 \\ https://hal.science/hal-02551151}

Submitted on 22 Apr 2020

HAL is a multi-disciplinary open access archive for the deposit and dissemination of scientific research documents, whether they are published or not. The documents may come from teaching and research institutions in France or abroad, or from public or private research centers.
L'archive ouverte pluridisciplinaire HAL, est destinée au dépôt et à la diffusion de documents scientifiques de niveau recherche, publiés ou non, émanant des établissements d'enseignement et de recherche français ou étrangers, des laboratoires publics ou privés. 


\section{Transition entre les cultures néolithiques de Sesklo et de Dimini :} les catégories céramiques

Jean-Paul Demoule, Kostas Gallis, Laurence Manolakakis

\section{Citer ce document / Cite this document :}

Demoule Jean-Paul, Gallis Kostas, Manolakakis Laurence. Transition entre les cultures néolithiques de Sesklo et de Dimini : les catégories céramiques. In: Bulletin de correspondance hellénique. Volume 112, livraison 1, 1988. pp. 1-58;

doi : https://doi.org/10.3406/bch.1988.1737

https://www.persee.fr/doc/bch_0007-4217_1988_num_112_1_1737

Fichier pdf généré le 19/04/2018 


\section{Résumé}

Les données des fouilles récentes de l'Éphorie de Larissa (Plateia Magoula Zarkoit, Makrychori 2) ainsi qu'un réexamen statistique des fouilles de V. Milojcic (Arapi, Otzaki, entre autres) ont permis de préciser la transition entre la culture néolithique thessalienne de Sesklo et celle de Dimini. II s'agit, à l'échelle de l'ensemble de la péninsule balkanique, d'un moment de transformation historique majeure. Les analyses montrent en fait que seule l'apparition d'une céramique noire polie est un élément réellement nouveau. Pour les autres catégories de céramique, on peut montrer, tant dans les formes que dans les décors ou le techniques, qu'elles résultent d'expérimentations et d'évolutions technologiques progressive', issues de la culture de Sesklo. On pourrait ainsi distinguer successivement une phase Sesklo tardive, une phase de transition dite "de Zarko», une première phase Dimini dite "Larissa» (au sens redéfini ici) et une seconde phase dite " Tsangli ». Ce moment de transition se caractéri» néanmoins par une baisse quantitative de la céramique fine, monochrome ou décorée.

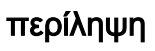

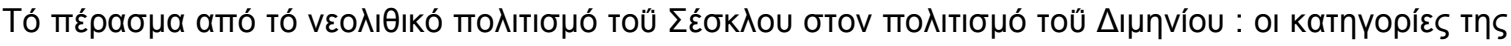

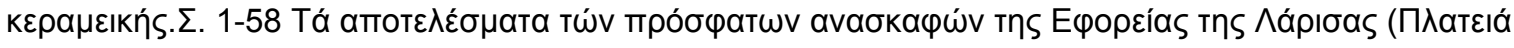

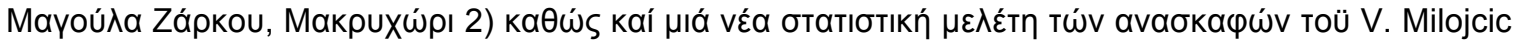

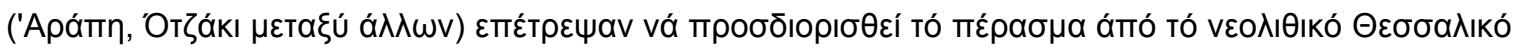

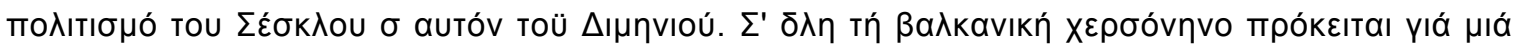

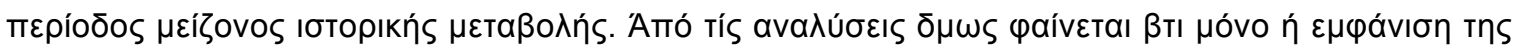

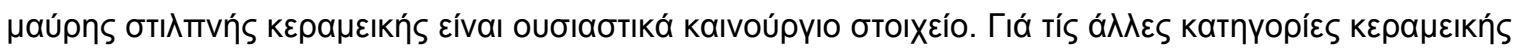

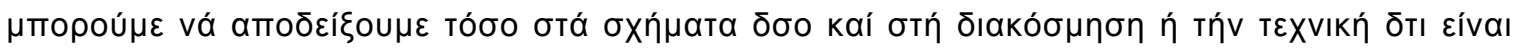

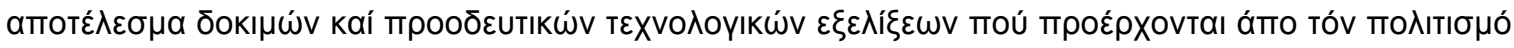

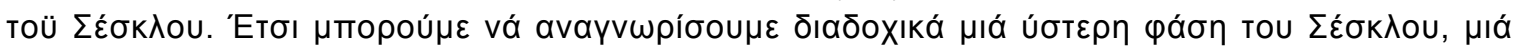

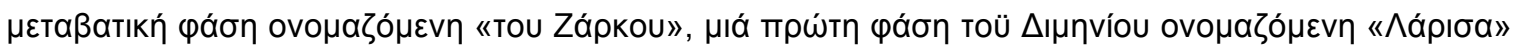

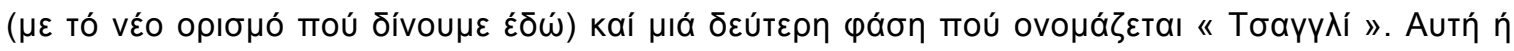

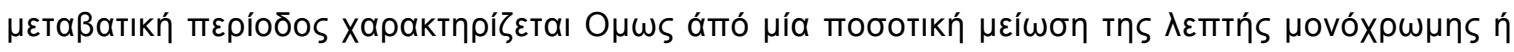

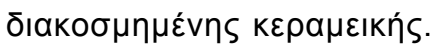




\section{TRANSITION ENTRE LES CULTURES NÉOLITHIQUES DE SESKLO ET DE DIMINI : LES CATÉGORIES CÉRAMIQUES}

La présente contribution est le premier volet d'une étude sur le Néolithique thessalien; elle est consacrée à la définition du cadre typologique et chronologique de la fin de la culture de Sesklo et du début de celle de Dimini. Le volet suivant portera plus spécifiquement sur l'apport et l'interprétation historique et socio-économique des analyses physico-chimiques effectuées sur le corpus céramique étudié. Ces recherches sont menées dans le cadre d'une mission archéologique franco-hellénique ${ }^{1}$.

\section{HISTORIQUE DES RECHERGHES ET RAPPEL DE LA PROBLEMATIQUE.}

Vers le milieu du cinquième millénaire, dans le calendrier du radiocarbone non calibré, le néolithique de la péninsule balkanique est marqué par une forte rupture. D'un

(1) Cette mission se déroule depuis 1984, avec le soutien de l'Éphorie des Antiquités Préhistoriques et Classiques de Larissa, du Ministère grec de la Culture, de la Sous-Direction des Sciences Sociales et Humaines du Ministère français des Affaires Étrangères et de l'École française d'Athènes ainsi que, en 1984, de l'Université de Crète. Outre les responsables (K. Gallis, Éphorie de Larissa ; C. Perlès, Université de Paris X et URA no 28 du CNRS; J.P. Demoule, Université de Paris 1 et URA no 12 du CNRS), y ont notamment participé N. Lianeris (Université de Crète, 1984), L. Manolakakis (Université de Paris I), D. Petermüller (Université de Paris I), D. Reduron (URA 28 du CNRS), F. Thiercellin (Université de Paris I), K. Vitelli (Université d'Indiana, Bloomington), ainsi que le personnel technique de l'Éphorie de Larissa. Le réexamen des matériaux déjà publiés issus des fouilles menées naguère en Thessalie par V. Milojçić a bénéficié de l'autorisation du Professeur H. Hauptmann (Université de Heidelberg), que les auteurs tiennent à remercier vivement. Les études physico-chimiques sont menées par H. Knoll et G. Schneider (Université de Berlin, avec l'appui de la Fondation Volkswagen). Les auteurs remercient ces différents organismes et leurs responsables pour leur soutien institutionnel et financier. Ils remercient également G. Toufexis, archéologue, $\mathrm{Ch}$. Samaras, artiste-peintre, P. Genas, dessinateur, de l'Éphorie des Antiquités de Larissa, pour leur assistance dans la réalisation et la mise au point des documents graphiques. Ils remercient enfin tout particulièrement D. Houliaras, T. Tloupas, K. Theodoropoulos, archéologues amateurs, à l'activité desquels sont dus un certain nombre de sites figurant sur la carte 1 , ainsi qu'une partie du matériel céramique analysé ici. 
point de vue typologique, on voit partout les céramiques fines peintes, aux formes usuellement curvilignes, faire place à une céramique noire peu ou non ornée, aux profils nettement carénés. D'un point de vue économique, le nombre des sites tend à s'accroître, des régions non encore occupées sont maintenant habitées (Macédoine centrale et orientale et Thrace grecques; colonisation de l'Europe tempérée par la "Céramique Linéaire" à partir du Nord des Balkans), l'élevage des bovins tend à supplanter celui des ovicapridés. D'un point de vue historique, en Grèce notamment, des sites anciens sont abandonnés tandis que de nouveaux sont fondés et l'on observe aussi, comme à Servia, Sesklo ou Tsangli, d'importantes couches de destruction. En même temps, des parallèles typologiques ont pu être proposés entre cette nouvelle céramique noire polie, parfois décorée d'incisions, et certains sites anatoliens (TiTov [1969]; Lichardus et alii [1985], p. 261, 339). Ces transformations s'observent en Thessalie (Dimini ancien), mais aussi en Grèce centrale (Élatée) et méridionale, régions néanmoins mal connues pour ces périodes, ainsi qu'en Macédoine (Néolithique Moyen de Dikili Tach, Sitagroi I-II, Paradimi I-III, Vassilika, Akropotamos, etc.) et dans l'ensemble des Balkans plus généralement, avec les groupes de Cakran, Karanovo III - Veselinovo, Bălgărčevo, Topolnica, (Vraca), Cernica, Boian-Bolintineau, Vinča-Turdas, Danilo-Kakanj, pour ne citer que les principaux. On appelle traditionnellement "Néolithique Moyen" dans l'ensemble de l'Europe balkanique ce nouvel horizon (Lichardus et alii [1985]), même si les chercheurs utilisent en Grèce le terme de "Néolithique Récent" (Late Neolithic), pour des raisons qui tiennent à l'histoire de la recherche (cf. infra). Bien que ceci n'entre pas dans notre présent propos, on doit cependant souhaiter que l'on aboutisse prochainement, pour traiter du Néolithique européen, à une terminologie uniforme.

L'existence d'un horizon ancien de la culture de Dimini n'avait en effet pas été mise en évidence par les premières fouilles thessaliennes de Tsountas puis de Wace et Thompson. Mais celles-ci avaient cependant révélé la présence d'une céramique peinte mate (malt painted), reconnue également lors des recherches ultérieures de Mylonas, Grundmann et Schachermeyr. C'est avec le sondage stratigraphique d'Arapi, et les diverses fouilles menées en Thessalie par Vl. Milojčić qu'une détermination plus exacte de cet horizon put être effectuée (Hauptmann et Milojčıć [1969]). Nėanmoins, ces travaux comportaient un certain nombre de limitations, que les recherches menées depuis lors ont permis de lever en partie. Il s'agissait notamment des points suivants :

a) D'un point de vue stratigraphique, la séquence du site d'Arapi ne comportait ni les niveaux immédiatement inférieurs (les couches 8-9 sous-jacentes sont attribuables à la culture de Pré-Sesklo-Magoulitsa, et Sesklo proprement dit n'est pas représenté), ni les niveaux immédiatement postérieurs (Otzaki), dont les couches ont été détruites. C'est pourquoi le récent sondage de Plateia Magoula Zarkou (cf. infra) est désormais le premier à offrir une séquence claire entre Sesklo et Dimini Ancien, et à permettre d'étudier, ou du moins de poser le problème d'une éventuelle transition - l'idée d'une rupture brutale étant plus communément admise. Par ailleurs, les sondages de Makrychori 2 et de Soufli Magoula présentent, comme Aghia Sofia (ce dernier site n'étant encore que préliminairement publié : MıLoJčı́́ et alii [1976]), une continuité entre les phases anciennes ("Tsangli " et "Arapi ») et les phases récentes ("Aghia Sofia ", "Otzaki ", "Dimini Classique", ou encore "Otzaki A, B, C.») de la culture de Dimini.

b) D'un point de vue chrono-culturel, les recherches sur cette période étaient grevées par la "culture de Larissa", définie par Vl. Milojčić malgré l'absence d'évidences 
stratigraphiques claires, et fréquemment remise en question depuis lors (par exemple Holmberg [1964], Treuil [1983], Lichardus et alii [1985], Hauptmann [1986], etc). La stratigraphie de Plateia Magoula Zarkou a donc également permis de démontrer que la "culture de Larissa" n'était que l'une des variétés céramiques de la phase Tsangli, phase que l'on peut désormais plus opportunément dénommer "Tsangli-Larissa" (Gallis [1987]).

c) Les études avaient été menées en l'absence d'analyses physico-chimiques, dont il était donc nécessaire de tester l'utilisation, mème si l'homogénéité des argiles limoneuses de la plaine thessalienne en limitait en partie la portée. De telles études étaient particulièrement nécessaires pour la mise en évidence d'éventuelles relations d'échange.

d) Les progrès inévitables des méthodes de fouille et d'analyse réalisés dans les deux dernières décennies permettaient également d'apporter de nouvelles données, notamment du point de vue de l'outillage lithique, des sciences naturelles et des techniques statistiques. De même, les fouilles menées entre temps dans les régions voisines permettaient également d'élargir la problématique.

La présente étude conserve néanmoins un caractère préliminaire, dans la mesure où des publications monographiques plus détaillées devront suivre ultérieurement, où certains sondages thessaliens restent encore presqu'entièrement inédits (région de Volos et de Karditsa) et où les fouilles elles-mêmes, notamment à Plateia Magoula Zarkou, ne sont pas toutes achevées. C'est donc le nouveau cadre d'étude et les nouvelles directions de recherche qui seront présentés ici.

\section{LE CORPUS : LES SITES ET LA MÉTHODE.}

\subsection{La méthode.}

On a souhaité ici reprendre sur des bases statistiques le problème des évolutions stylistiques. La typologie a surtout porté sur les grandes catégories céramiques et les techniques de décor, sans entrer dans le détail des formes de récipients et des thèmes ornementaux. Certaines difficultés de définition ont eu trait à ces techniques elles-mêmes, parfois particulièrement malaisées à restituer (décor "frotté " notamment), ce qui n'est normalement pas le cas dans le Néolithique européen, et certains types étant peu standardisés. La description s'est bornée ici à une approche macroscopique, les analyses physico-chimiques menées par H. Knoll et G. Schneider (Université de Berlin) et dont il sera rendu compte dans la prochaine livraison de ces études, nécessitant précisément une définition archéologique préalable des groupes céramiques. Il en va de même pour les expérimentations effectuées par K. D. Vitelli (Université d'Indiana).

On a procédé, suivant les sites, à des échantillonnages, lorsque le matériel était plus abondant qu'il n'était nécessaire pour le but proposé ; ceci sera précisé ci-dessous à propos de chacun des sites étudiés (fig. 1). La plupart des décomptes ont été fait par poids plutôt que par effectifs, ces deux approches étant sensiblement comparables et le poids étant d'un maniement plus souple. L'essentiel des traitements a consisté en histogrammes de pourcentage et en tris croisés des catégories céramiques par couches archéologiques, des méthodes de classification plus complexes n'ayant pas paru justifiées à ce stade. 


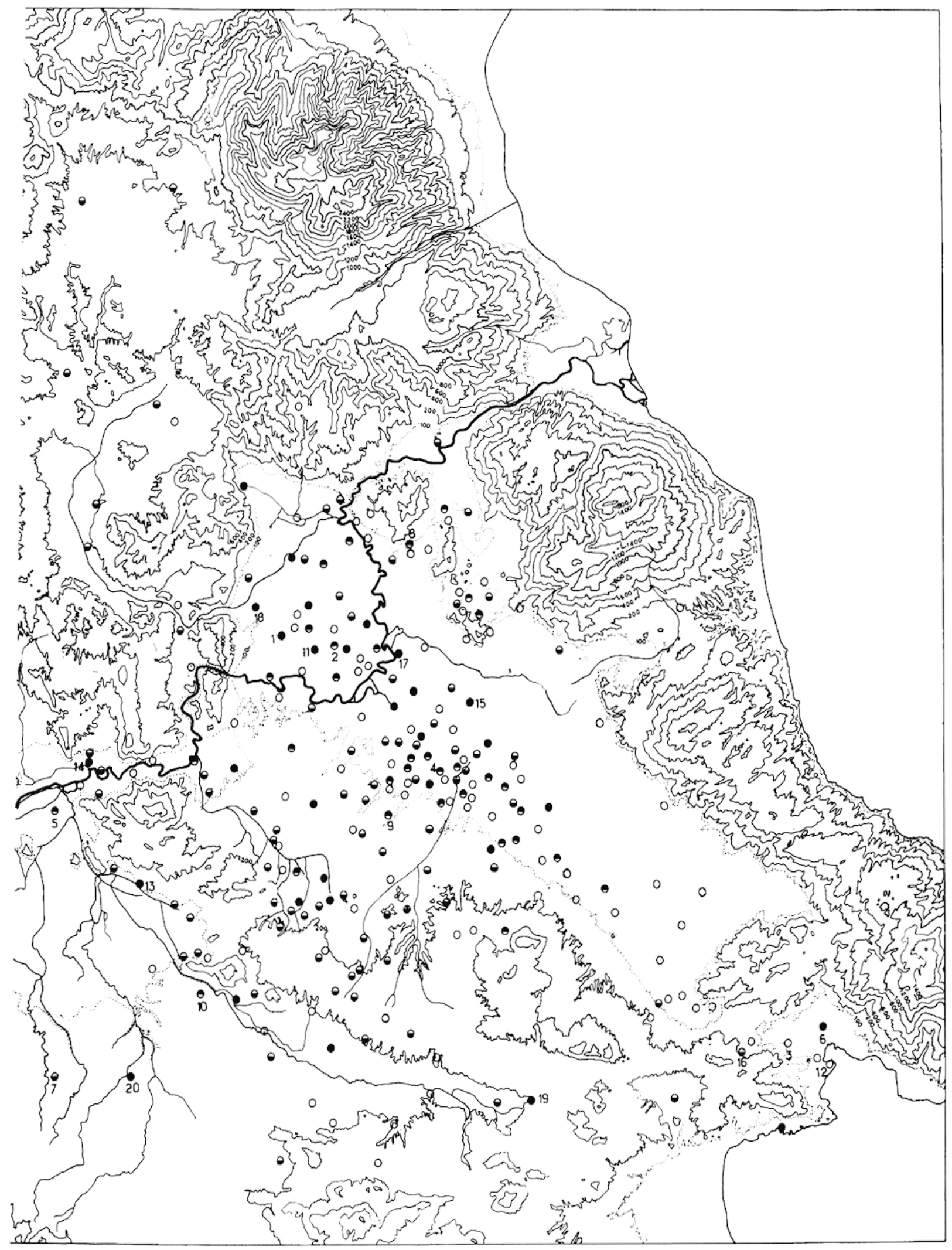

Fig. 1. - Carte des sites des périodes Sesklo et Tsangli-Larissa en Thessalie orientale.

LÉGENDE : $\bullet$ sites occupés pendant la période Sesklo;

- sites occupés pendant la période Tsangli-Larissa;

- sites occupés pendant les périodes Sesklo et Tsangli-Larissa ;

- sites néolithiques appartenant à d'autres périodes;

1: Aghia Sofia; 2 : Arapi; 3 : Dimini; 4 : Kardara Magoula; 5 : Keramidi; 6 : Koufovouno; 7 : Magoula Aghiou Georghiou, Sophades; 8 : Makrychori 2; 9 : Nikaia; 10 : Orphana; 11 : Otzaki; 12 : Pefkakia; 13 : Petrino; 14 : Plateia Magoula Zarkou ; 15 : Platykampos; 16 : Sesklo ; 17 : Soufli Magoula ; 18 : Tsalmas; 19 : Tsangli; 20 : Tsani. 


\subsection{Les sites.}

2.2.1. Plateia Magoula Zarkou. Cette fouille a renouvelé la problématique de la "culture de Larissa". Elle a été entreprise en 1976 afin de résoudre le problème de la position stratigraphique de la céramique "Larissa" associée dans un cimetière néolithique à crémation à la céramique "gris-sur-gris" classique de la période Tsangli. Ce cimetière se trouvait en effet à $300 \mathrm{~m}$ au Nord d'une "magoula" typique, dont le sommet, aplani par des remaniements ultérieurs de l'Âge du Bronze, justifiait l'appellation de "Plateia Magoula". Le sondage de $8 \times 5 \mathrm{~m}$ effectué au sommet de la magoula fut poursuivi en $1981,1983,1984$ et 1987, date à laquelle il atteint la nappe phréatique à $-8,2 \mathrm{~m}$, ce qui entrave la poursuite de la fouille. La surface actuelle de la plaine thessalienne s'est en effet exhaussée, par suite de l'alluvionnement, d'un à deux mètres depuis le Néolithique, et la nappe phréatique de même. Le sol vierge n'a donc pas été encore trouvé. Au-dessous de $-5,1 \mathrm{~m}$, les couches contiennent le matériel décoré typique des phases récentes de Sesklo (décor "flammé», décor "frotté»), tandis qu'entre 5,1 et $4 \mathrm{~m}$, les niveaux appartiennent au "Néolithique Récent", plus précisément à ce que cette fouille et celle de Makrychori 2 (ci-dessous) permettent de nommer "période de Tsangli-Larissa". En effet, on l'a vu, ces deux fouilles ont permis d'établir définitivement que la céramique Larissa
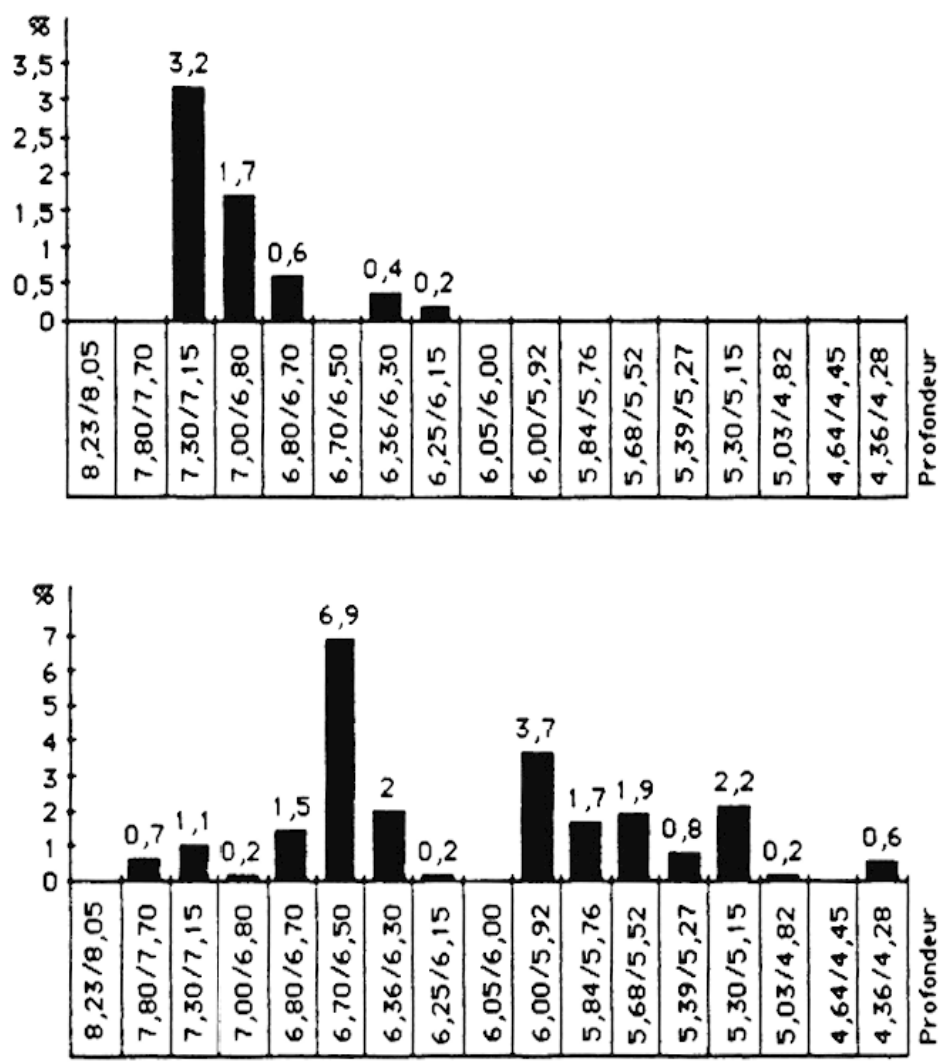

TAbleau 1. - Evolution stratigraphique (en poids) de la céramique peinte à décor brun ou rouge sur fond clair, sur le site de Plateia Magoula Zarkou; variante "classique et variante "dégénérée". 


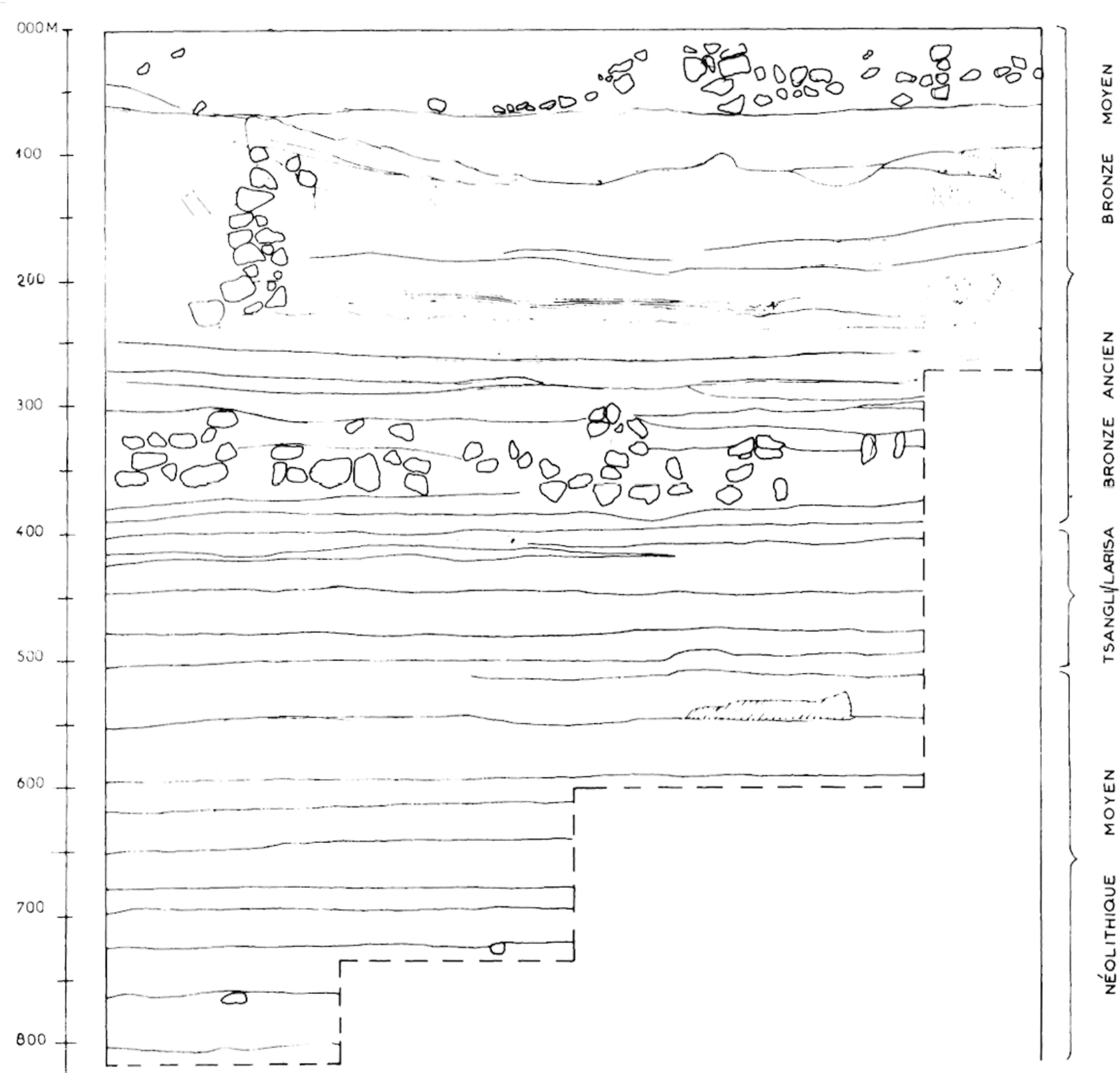

Fig. 2. - Coupe stratigraphique de Plateia Magoula Zarkou.

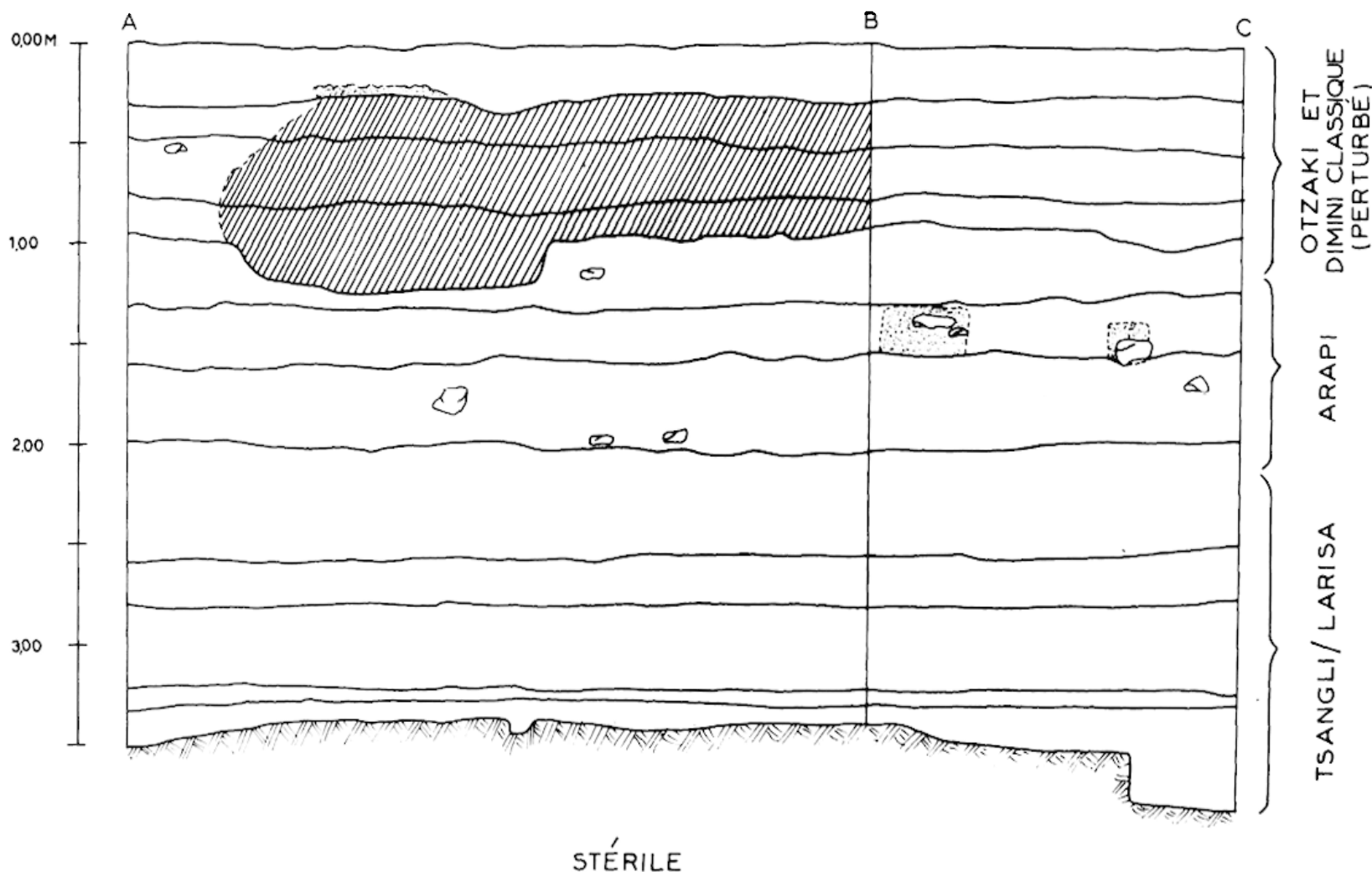

Fig. 3. - Coupe stratigraphique de Makrychori 2. 
noire polie, avec toutes ses variantes, se trouvait sur ces sites associée à la céramique typique de la période Tsangli, et pendant toute la durée des couches concernées (Gallis [1987]).

Après cette période, le site, au moins dans la partie fouillée, reste inhabité jusqu'au début du Bronze Ancien, comme le montre la couche d'humus d'une vingtaine de centimètres trouvée à $-4 \mathrm{~m}$. Les derniers 4 mètres appartiennent au Bronze Ancien et Moyen (fig. 2). Une grande fosse du Bronze Ancien a perturbé les différents niveaux sousjacents dans la partie Est du sondage jusqu'à la profondeur de $-5,3 \mathrm{~m}$, mais la partie Ouest est restée heureusement intacte.

Les matériaux décomptés ici représentent un échantillon d'un tiers du total découvert, régulièrement prélevé tout au long de la stratigraphie, représentant $178.300 \mathrm{~g}$ de céramique; aucun tri préalable n'avait été effectué à la fouille. L'ensemble de la céramique a par ailleurs été examiné.

2.2.2. Makrychori 2. Cette fouille a été menée en novembre 1981 avec le même objectif qu'à Plateia Magoula Zarkou : clarifier la position stratigraphique de la céramique Larissa noire polie (Gallis [1987]). Ce tell ne mesure que $3 \mathrm{~m}$ de haut; il n'a été découvert que récemment et se trouve à $1,5 \mathrm{~km}$ à l'Ouest du site éponyme de Rachmani, situé sur la même commune. Un sondage de $4 \times 2 \mathrm{~m}$, orienté Est/Ouest a été conduit jusqu'au sol vierge, à une profondeur de $1,5 \mathrm{~m}$. Les niveaux inférieurs, de 3,5 à $2,2 \mathrm{~m}$, appartiennent à la période Tsangli-Larissa. La céramique noire lissée Larissa accompagne tout au long de cette couche la céramique à peinture mate et la céramique grise - cette dernière moins abondante qu'à Plateia Magoula Zarkou. Au moins trois sols d'habitat peuvent être distingués durant cette période (fig. 3). On trouve ensuite, de 2,2 à 1,2 $\mathrm{m}$ des niveaux Arapi. Au-dessus, des niveaux contenant de la céramique Otzaki et Dimini Classique avaient été perturbés. Les résultats de Makrychori 2 complètent et confirment ceux de Plateia Magoula Zarkou, dans la mesure où, sur ce site, la période Tsangli-Larissa est suivie sans interruption ni perturbations par des niveaux Arapi. Comme à Plateia Magoula Zarkou, les décomptes effectués ici à partir des poids reposent sur un échantillonnage représentatif de $31.595 \mathrm{~g}$ de céramique de toutes catégories, sans tri à la fouille.

2.2.3. Otzaki-Magoula. Ce site, fortement perturbé par des travaux militaires de la guerre balkanique, a fait l'objet entre 1953 et 1958 de la part de V. Milojčić de deux sondages sur environ $335 \mathrm{~m}^{2}$, et d'un nettoyage de coupe. Il n'existe pas de stratigraphie continue de la surface jusqu'au sol vierge. Dans la "Fläche II" ont été atteints des niveaux du néolithique ancien (Proto-Sesklo et Pré-Sesklo). Dans les "Fläche I-II", $4 \mathrm{~m}$ de niveaux Sesklo avec 8 horizons d'habitat, recoupés dans les niveaux supérieurs par des fosses Dimini, ont été fouillés (Milojčıć et Mılojčıć-von Zumbusch [1983]; Motrier [1981]). Ce sont ces niveaux qui ont été repris ici, et qui ont fait l'objet d'un décompte, portant sur 1.016 fragments de céramique décorée - la totalité de la céramique n'ayant pas été conservée après la fouille (tableaux 2 et 3). Enfin, à un autre emplacement du tell, le "grand profil" concerne des couches de Dimini ancien et récent.

2.2.4. Arapi. Ce site, également perturbé par les travaux militaires de la guerre balkanique, a fait l'objet, en 1955 et de la part de V. Milojčić, de deux nettoyages de coupes, précisément à partir de ces tranchées de défense qui avaient profondément entaillé le tell. La stratigraphie (Pré-Sesklo, Tsangli, Arapi) d'environ $2 \mathrm{~m}$ a servi de 


\begin{tabular}{|c|c|c|c|c|c|c|c|c|c|c|c|}
\hline & \multicolumn{11}{|c|}{ Planum } \\
\hline & II $16 \mathrm{ab}$ & II 13-15 & II $10-12$ & II $8-9$ & II $6-7$ & II-5, I-8 & II- $-4, \mathrm{I}-7$ & $\mathrm{II}-3, \mathrm{I}-6$ & II- $-2, \mathrm{I}-5$ & $5 \mid 4,4-5$ & I1-3 \\
\hline Cér. imprimée ..... & 70,7 & 61 & 36,4 & 14,9 & 6,1 & 2,9 & 3,7 & 5,1 & 5,2 & 1,8 & 2,9 \\
\hline Cér. incisée $\ldots \ldots \ldots \ldots \ldots \ldots$ & 12 & 0 & 0 & 0 & 0 & 0 & 0 & 0 & 0 & 0 & 0 \\
\hline Cér. imprimée et peinte & 1,5 & 0 & 0 & 4,6 & 4 & 1,4 & 1,9 & 7,6 & 0,7 & 3,5 & 0 \\
\hline Cér. décor plastique $\ldots \ldots \ldots \ldots$ & 9,8 & 7,3 & 5,2 & 1,1 & 1 & 1,4 & 0 & 0,8 & 0 & 3,5 & 0 \\
\hline Cér. Sesklo brun-rouge sur clair. & 3 & 18,3 & 23,4 & 24,1 & 26,3 & 43,5 & 74,1 & 48,3 & 45,2 & 49,1 & 45,7 \\
\hline Cér. décor frotté $\ldots \ldots \ldots \ldots \ldots$ & 5,3 & 11 & 35,1 & 55,2 & 62,6 & 50,7 & 20,4 & 39 & 46,7 & 38,6 & 23,8 \\
\hline Cër. «Proto-gris-sur-gris" . . . . . & 0 & 0 & 0 & 0 & 0 & 0 & 0 & 4,2 & 2,2 & 3,5 & 1 \\
\hline Cér. gris-sur-gris $\ldots \ldots \ldots \ldots$ & 0 & 0 & 0 & 0 & 0 & 0 & 0 & 0 & 0 & 0 & 4,8 \\
\hline Cér. peinte diverse .... & 0,8 & 2,4 & 0 & 0 & 0 & 0 & 0 & 0 & 0 & 0 & 12,4 \\
\hline Cér. noire lissée $\ldots \ldots \ldots \ldots \ldots$ & 0 & 1,2 & 0 & 0 & 0 & 0 & 0 & 0 & 0 & 0 & 9,5 \\
\hline
\end{tabular}

TABleau 2. - Pourcentages, par couches regroupees, des différentes catégories céramiques de la période Sesklo sur le site d'Otzaki; décomptes sur 1.016 fragments décorés.
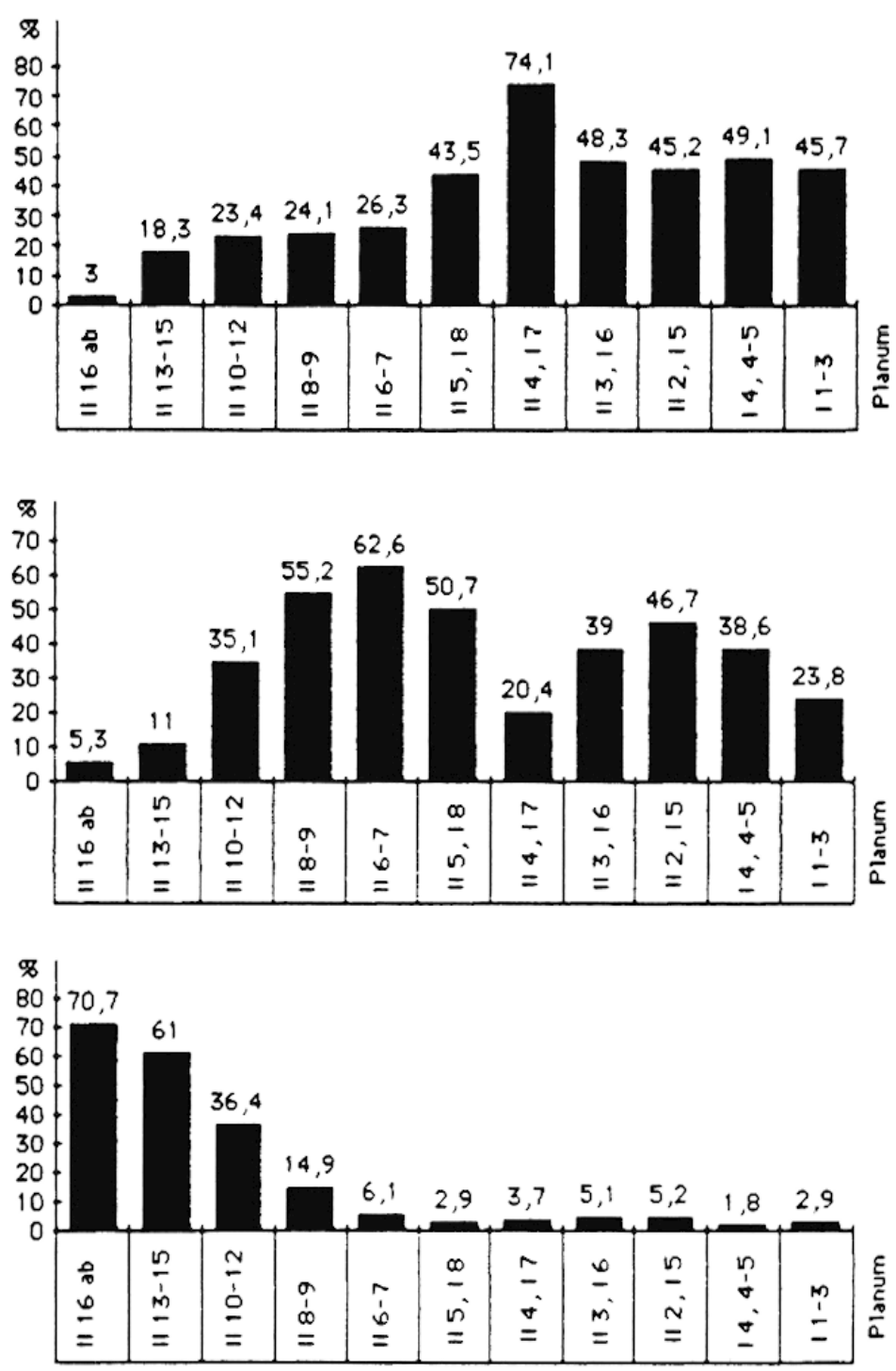

TABleau 3. - Évolution stratigraphique, sur le site d'Otzaki (cf. tabl. 2), de la céramique peinte en brunrouge, de la céramique à décor frotté et de la céramique à décor imprimé; décomptes sur 1.016 fragments décorés. 
référence pour la définition des deux premières périodes de la culture de Dimini (Hauptmann et Mrlojčıć [1969]). On a repris ici le matériel de ces fouilles (tableau 18). De même que pour le site précédent, les résultats valent essentiellement pour la céramique décorée, la totalité de la céramique non-décorée n'ayant pas été préservée. C'est pourquoi, si l'échantillon décompté représente $16.690 \mathrm{~g}$ de céramique, la céramique grossière encore conservée n'en représente qu'un tiers (tabl. 18a) alors que d'après la publication (HauptmanN et Milojčıć [1969], p. 96-114), elle en constituait sensiblement les deux tiers (tabl, $18 \mathrm{~b}$ ).

2.2.5. autres sites. Outre ces quatre sites, qui ont été étudiés de manière exhaustive à partir des matériaux primaires, on a procédé, sur certains mobiliers ou à partir des publications, à l'étude comparative de plusieurs autres sites. Mentionnons notamment Soufli Magoula où, outre une petite nécropole à incinération de la période Proto-Sesklo (Gallis [1982]) puis de la période Tsangli-Larissa (BIEsantz [1959]), l'Éphorie de Larissa a pratiqué récemment un sondage de contrôle; Sesklo, dont la céramique décorée vient de faire l'objet d'une analyse formalisée toute récente, portant sur environ 500 tessons (Kotsakis [1983]; cf. aussi WiJnen [1982]); et divers sondages, comme Tsapocha, Tsani, Tsangli, Nessonis, etc. (Theocharis [1981], Wace et Thompson [1912]). Par ailleurs, d'importants matériaux collectés lors de prospections de surface (cf. note 1) ont également été examinés, notamment ceux provenant des sites de Keramidi, Orphana, Tsalmas, etc.

\section{LES CATÉGORIES DE LA CÉRAMIQUE.}

\subsection{Les catégories issues de la culture de Sesklo.}

3.1.1. La céramigue peinte Sesklo en brun ou rouge sur fond clair (A3 $\alpha-\gamma$, fig. 4 et 18 ).

3.1.1.1. Décors el formes. Il s'agit du "fossile-directeur" par excellence de la culture de Sesklo. Wace et Thомpson (1912) ont distingué un style peint en blanc sur fond rouge (A3 $\alpha$ ), un style peint en brun-rouge sur engoble blanc brillant (A3 $\beta$ ) et un style peint en brun-rouge sur fond clair non recouvert d'engobe (A3 $\gamma$ ). Le premier style ne s'est pas rencontré dans le corpus. Considéré comme relativement ancien, il ne semble pourtant attesté sur le site éponyme de Sesklo que dans la couche la plus récente (KoTsa Kis [1983], p. 97). La peinture blanche sur rouge, avec notamment des motifs de chevrons continus exécutés en plusieurs lignes parallèles, caractérise plus au Nord (Yougoslavie, Bulgarie) des phases relativement anciennes du Néolithique Ancien.

La thématique des deux autres styles, analogues à l'engobe près, utilise également de grands chevrons faits de lignes parallèles et courant tout autour du récipient. Ces chevrons peuvent être eux-mêmes bordés de rangées de triangles et délimiter des surfaces remplies par des motifs intermédiaires. Quant à ces triangles et motifs intermédiaires, on a fréquemment distingué entre un style "solide" ou compact ("solid style»), avec des motifs rectilignes, souvent "en escalier" (fig. $4, \mathrm{n}^{\circ} 2$ ); et un style "flammé", avec des motifs aux contours plus curvilignes (fig. $4, n^{0} 4$ ). Les deux se distinguent du "style linéaire", où seules les lignes en chevrons sont présentes. D'après les chronologie usuelles (Theocharis [1981]), le style linéaire serait le plus récent, et serait lui-même suivi d'une 

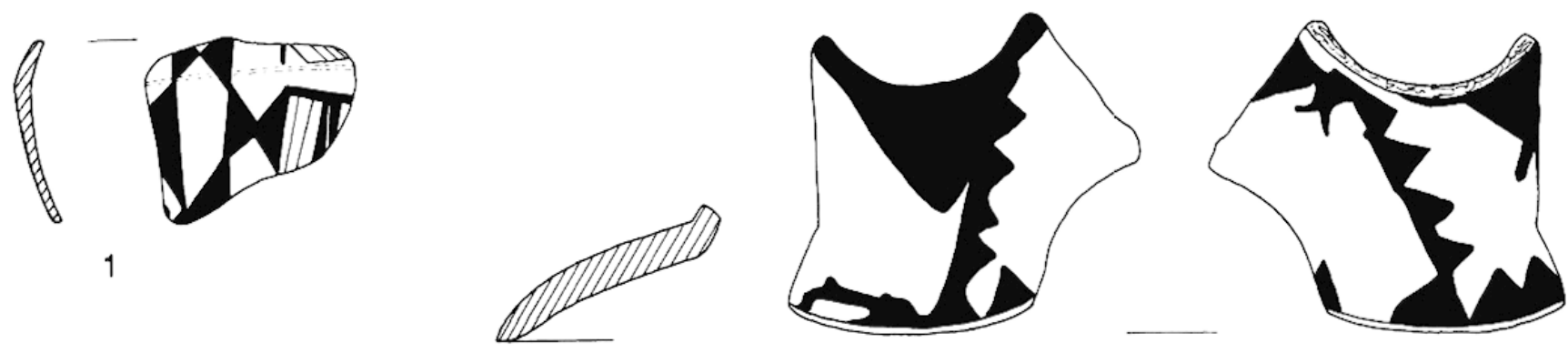

2
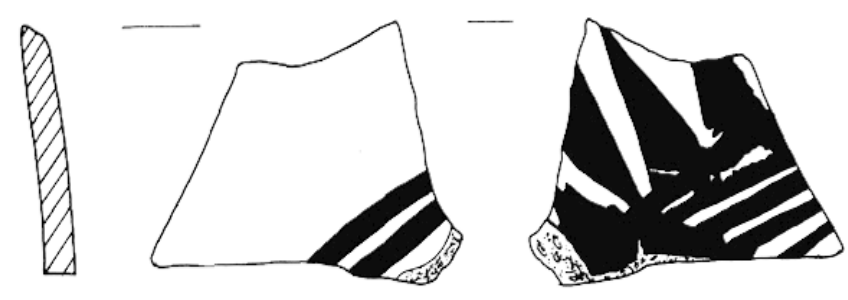

3
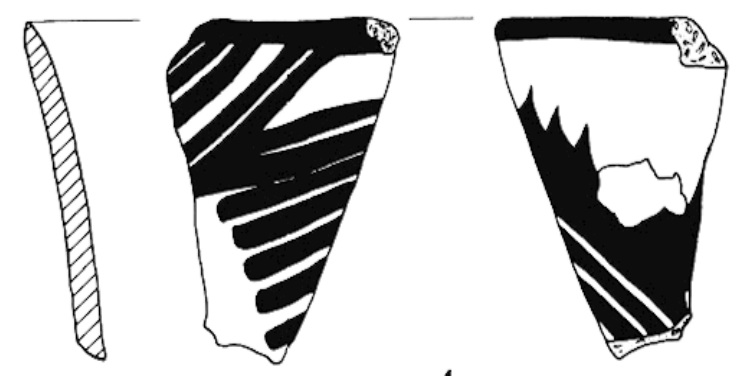

4

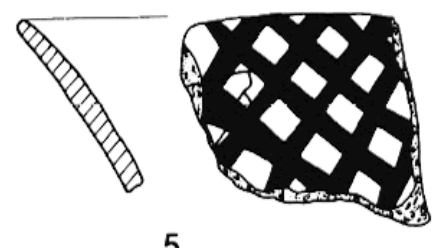

5

Fig. 4. - Céramique peinte en brun-rouge sur clair (Plateia Magoula Zarkou). Env. $1: 3$.

période dominée essentiellement par un autre type de décor, le décor dit "frotté» (cf. infra, § 3.1.2.). C'est du moins la situation en Thessalie de l'Ouest et du Sud, notamment à Tsani, où la céramique à "décor frotté" n'est cependant pas attestée (WACE et Tномpson [1912]), à Tsangli (idem), à Sesklo (Kotsakis [1983], p. 78-92 et 97-98). La situation paraît en revanche plus nuancée en Thessalie du Nord-Est, où à Otzaki la céramique à décor frotté accompagne dès le début la céramique peinte en brun sur blanc (tabl. 3), tandis que la succession solide/linéaire n'est pas apparente (MotTier [1981]). Quant aux formes, il s'agit essentiellement de jattes caractéristiques, à fond plat et à parois hautes et concaves (tabl. 11); ce peu de variété indique une association étroite entre style et fonction et il s'agit sans doute d'une vaisselle dont l'utilisation possède une signification sociale. Il existe quelques formes particulières et très minoritaires, notamment une sorte de bouteille à colerette présente, à Plateia Magoula (fig. $4, \mathrm{n}^{\circ} 2$ ) comme à Otzaki (Motrier [1981], pl. 39, 9;44,4), dans des niveaux récents.

En fait, dans le corpus dont on dispose, ce style classique est fort peu représenté à Plateia Magoula Zarkou (tabl. 1); il est plus abondant à Otzaki, où il représente souvent la moitié de la céramique décorée (tabl. 2-3). Par comparaison, il représente dans presque toutes les couches du site de Sesklo la totalité de la céramique décorée, c'est-à-dire un 

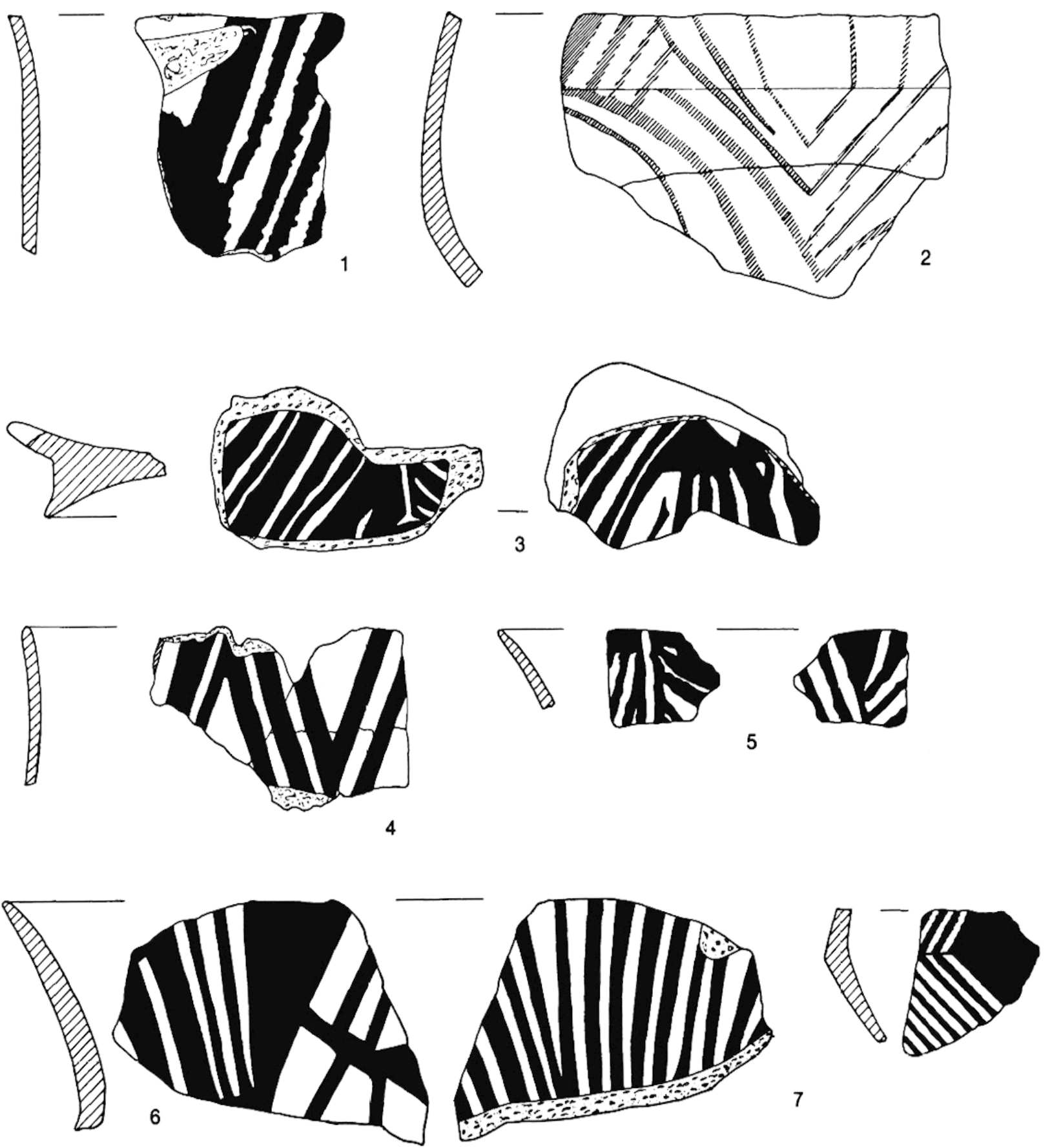

Fig. 5. - Céramique à décor frotté (Plateia Magoula Zarkou). Env. 1:3. 
pourcentage de 2 à $3 \%$ du total céramique dans les couches les plus anciennes, de 10 à $20 \%$ dans les couches plus récentes (Koтsakis [1983]). Notons que ce dernier site montre fréquemment de sensibles variations intra-site pour une même phase d'occupation, ce qui doit nuancer les conclusions portant sur des sondages limités. On doit par ailleurs distinguer, à côté des exemplaires usuels, un style qu'on appellera "dégénéré". Proche des précédents, il s'en distingue par une technique beaucoup moins soignée. L'engobe paraît le plus souvent absent (cf. catégorie A3 $\beta$ ). La peinture, en fonction de la cuisson, varie du rouge clair au brun sombre, voire au noir. Les thèmes du décor diffèrent sensiblement et se composent surtout de motifs en arêtes de poisson, qui évoquent beaucoup plus les thèmes de la céramique "à décor frotté" (infra § 3.1.2.).

3.1.1.2. Position chronologique. Quant à la périodisation, outre l'antériorité déjà mentionnée du style solide sur le style linéaire, on a proposé à partir d'un des sondages d'Otzaki une tripartition de la séquence Sesklo, concernant les formes et le décor de la céramique (MıLoJč́ćc [1959], Motrier [1981]). Un réexamen quantifié de ces données nous a permis de préciser l'évolution des catégories de céramique décorée (tabl. 2-3). La stratigraphie de Plateia Magoula (tabl. 5) montre également que la céramique à décor frotté n'est pas un phénomène tardif. La faiblesse quantitative sur ce site de la céramique peinte en brun sur blanc, parfois presque absente, peut tenir soit à la variabilité intersites, soit à une fonction particulière et restreinte, susceptible de ne pas être présente dans tous les points d'un habitat. La stratigraphie de Plateia Magoula montre enfin que la variante $A 3 \beta$ n'est définitivement plus attestee vers $6,3 \mathrm{~m}$, tandis que notre variante "dégénérée" reste seule présente, pour disparaître elle-même totalement entre $5 \mathrm{~m}$ et $4,5 \mathrm{~m}$ (tabl. 1). On a des raisons de penser que les nouveaux styles à peinture rouge qui se développent dans le même temps sont au moins partiellement la continuation de cette évolution stylistique (cf. infra § 3.3.1.3.).

\subsubsection{La céramique "À décor frotté" (fig. 5-6, 19).}

3.1.2.1. La lechnique du décor "frollè". Il n'existait pas jusqu'à présent de traduction française pour le décor dénommé et décrit d'abord par WACE et THомpson (1912), p. 1415 , sous le terme de "scrapped ware", terme traduit en grec par "xesti" et en allemand par "ausgewischte Ware». Sous sa forme "classique", il s'agirait d'une surface d'abord enduite uniformément d'un engobe, lequel est ensuite retiré à certains emplacements, délimitant ainsi des motifs "en négatif". Wace et Thompson distinguaient d'ailleurs plusieurs variétés : A3 $\delta$, ou "de Lianokladi», où l'on superpose puis retire par grattage à l'endroit des motifs ces deux couches successives, la couche inférieure étant blanche et le grattage faisant donc réapparaître le fond proprement dit cerné à chaque fois d'un liséré blanc; $\mathrm{A} 3 \varepsilon$, avec engobe sombre sur fond clair rouge pâle ou beige, faisant donc apparaitre des motifs en clair; et, symétriquement, $\mathrm{A} 3 \zeta$, avec un engobe rouge clair sur fond plus sombre, laissant donc apparaitre des motifs sombres sur clair. Cette typologie, ou du moins les deux dernières catégories, sont plus ou moins reprises par Y. Mottier dans sa publication déjà mentionnée des niveaux Sesklo d'Otzaki (Motrier [1981]); mais cette auteur n'examine pas la technique du décor.

Toutefois, une étude plus attentive de cette catégorie, y compris pour la céramique de ces niveaux d'Otzaki, montre que les techniques employées sont à la fois plus variées, mais aussi souvent malaisées à identifier. Plusieurs interprétations peuvent parfois être 

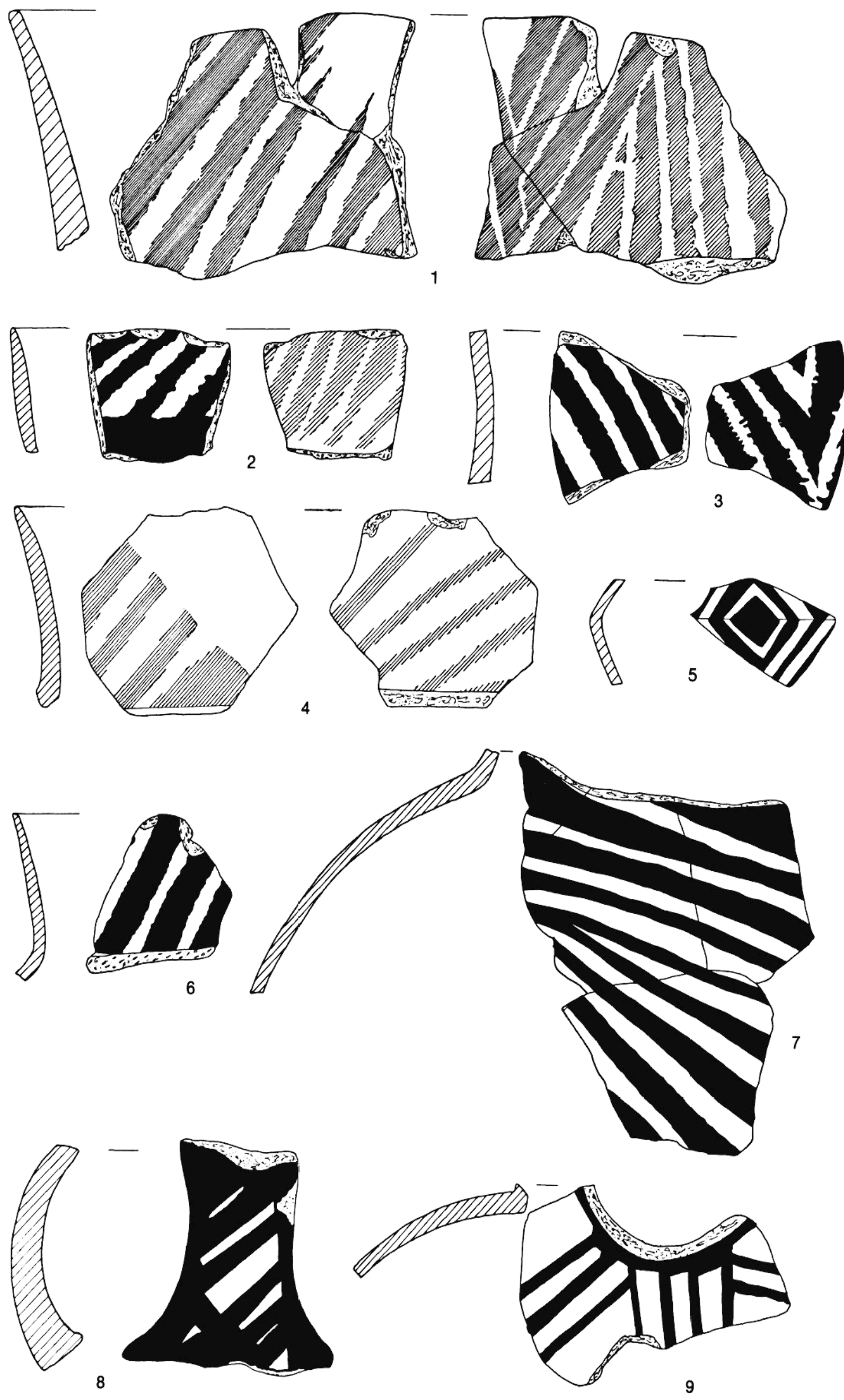

Fig. 6. - Céramique à décor frotté (Plateia Magoula Zarkou). Env. 1:3. 
proposées pour un même effet. On peut ainsi distinguer une série de choix techniques successifs, dont il n'est d'ailleurs pas certain qu'ils aient tous été réalisés (tabl. 4) :

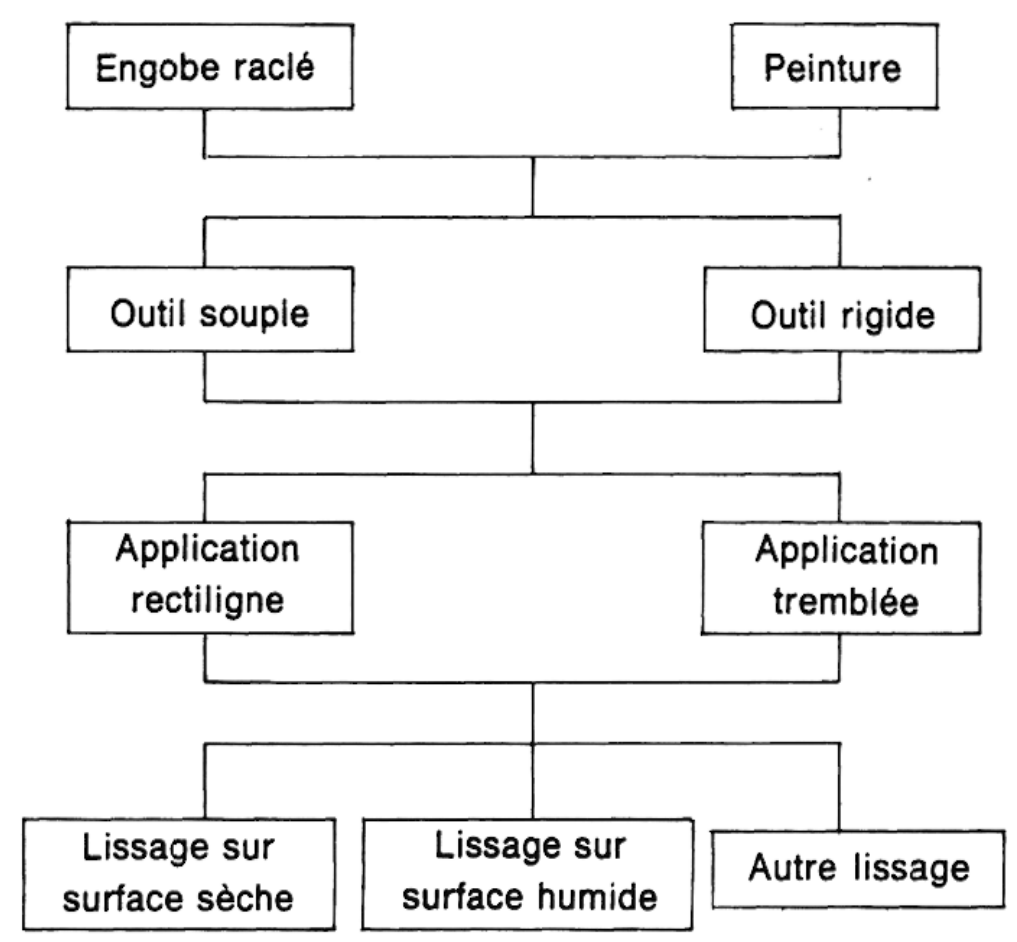

TaBleau 4. - Succession des choix techniques possibles pour la réalisation de la céramique à décor frotté.

a) L'engobe : à côté de la technique "canonique» précédemment décrite, il existe des cas où le même effet est indubitablement produit par des motifs directement peints en positif ; et des cas presque indistingables.

b) L'instrument : il faut distinguer, en cas de raclage, entre le raclage avec un outil rigide (spatule), laissant aux motifs des bords francs, et le raclage avec un outil souple (main, instrument souple), laissant des contours atténués; en cas de peinture, entre un pinceau rigide et un pinceau souple. L'apposition d'une peinture épaisse avec un pinceau relativement rigide peut créer un effet analogue à celui d'un raclage à la spatule, tout comme une peinture au pinceau souple par rapport à un raclage à l'outil souple.

c) Maniement de l'instrument : raclage ou peinture peuvent être effectués de manière franche, déterminant des segments de décor rectilignes, ou de manière "tremblée " (fig. $5, \mathrm{n}^{\text {" }} 1 ; 6, \mathrm{n}^{\text {os }} 1-3$ ).

d) Lissage : un lissage sur une surface encore humide créera également, quelle qu'ait èté la technique utilisée jusque-là, un aspect "tremblé" du décor, et atténuera aussi la netteté des contours, comme la possibilité d'identifier la technique - tandis que le lissage d'une surface déjà sèche se contentera de donner de la brillance à l'ensemble. Une autre forme de lissage semble avoir produit, dans de rares cas, un décor nettement en relief, formé d'étroits cordons lisses d'environ 3 à $4 \mathrm{~mm}$ de largeur et de moins d'un millimètre d'épaisseur. Plutôt qu'appliqués, ils semblent bien résulter d'un lissage intense avec un instrument rigide (spatule), qui aurait laissé subsister en négatif ces cordons composant le décor, et eux-mêmes lissés; il n'est pas exclu qu'ils aient pu parfois avoir été peints.

e) Cuisson : la cuisson semble avoir un effet important sur les nuances de couleur, et 
notamment sur leur variété. Si la dominante est brun-rougeâtre, et la cuisson normalement oxydante, cette céramique ne semble pas connaître le même degré de standardisation technique que la céramique peinte.

f) Érosion ultérieure : l'érosion ou l'effacement de la peinture (ou de l'engobe), consécutifs à l'utilisation puis à l'enfouissement, peuvent venir compliquer encore l'identification. La surface qui portait auparavant de la peinture apparaît comme moins lissée, plus mate. De même, une érosion partielle de la peinture offre un contraste entre la surface encore bien lissée du fond et la surface devenue mate de la peinture. La peinture (ou l'engobe "gratté"?) prend un aspect particulièrement croûteux et fragile quand les lignes du décor ne sont pas tremblées, ce qui tend à conforter l'hypothèse du lissage : lorsqu'il y a lissage, le décor est stabilisé mais prend un aspect tremblé, tandis qu'en l'absence de lissage, les contours des motifs restent plus ou moins rectilignes, mais la peinture, non stabilisée, tend à s'éroder.

L'arbre qui regroupe l'ensemble de ces données (tabl. 4) est en fait beaucoup plus un arbre des hypothèses qu'un arbre des techniques effectivement utilisées; il n'est en effet pas certain que toutes l'aient été. La technique du raclage de l'engobe est clairement apparente pour la variante dite "de Lianokladi" (A3 $\delta$ de Wace et Thompson), qu'on peut observer sur des vases provenant du site éponyme (Musée National, Athènes). Toutefois ce site de Lianokladi n'appartient plus géographiquement à la Thessalie mais à une petite plaine plus méridionale, celle de la vallée du Spercheios. L'unique tesson attribuable à cette catégorie identifié à Plateia Magoula Zarkou (à $-6,7 \mathrm{~m}$ ) confirme qu'il s'agirait bien d'une variante locale. Pour le reste, c'est-à-dire pour les variantes très majoritaires $\mathrm{A} 3 \varepsilon$ et $\mathrm{A} 3 \zeta$, l'utilisation du raclage de l'engobe comme technique de décor ne peut être que rarement affirmée avec certitude, tandis qu'il existe des cas indubitables de peinture. De fait, LeTsCh et Noll (1983), p. 138 disent avoir obtenu expérimentalement un résultat identique au "grattage" par une simple peinture sur surface humide, suivie d'un lissage. Les expériences menées par K.D. Vitelli dans le cadre du présent programme aboutissent aux mêmes conclusions (à paraître dans la suite de cette étude) et montrent, après peinture puis lissage sur une surface encore humide, le décor "tremblé" caractéristique. Il n'est donc pas certain que le terme anglais de "scrapped ware», qui évoque un "raclage" ou un "grattage", soit le terme qui convienne le mieux, pas plus d'ailleurs que le terme allemand d'"ausgewischte Ware", où l'on aurait "retiré par essuiement". Nous avons donc préféré ici celui, plus neutre, de "frotté", qui se réfère à l'aspect le plus souvent dilué des contours des motifs, sans prendre partie sur la technique réellement utilisée. Dans tous les cas, cette utilisation par les porteurs de la culture de Sesklo d'un décor finalement peu visible, et qui contraste singulièrement avec le décor peint en brun sur blanc, est un trait original dans le Néolithique européen.

3.1.2.2. Motifs et formes. "Raclée» ou "frottée», cette technique ne permet pas des thèmes décoratifs très complexes. Ceux-ci se bornent donc le plus souvent à des motifs couvrants en "arêtes de poisson" (fig. 5-6), un rapport à la surface à décorer peu structuré et sans zonation marquée du récipient, ce qui n'a rien à voir avec la céramique peinte en brun sur blanc, puisqu'il n'y a pas répétition linéaire d'un seul motif (le chevron) à l'intérieur d'une frise continue et délimitée, mais recouvrement de toute la surface du vase.

De fait, les formes sont également différentes. Il s'agit d'une part de bouteilles à panse arrondie et col court légèrement évasé (fig. $5, n^{\circ} 6 ; 6, n^{\circ 8} 6-9$ ); d'autre part de 
gobelets hauts de tailles diverses, à parois fines, légèrement évasés, souvent munis d'une large anse verticale en ruban (fig. $5, n^{\text {os }} 1-5$ ); les parois des exemplaires de petite taille n'excèdent pas $3 \mathrm{~mm}$. La pâte est bien micacée, la surface soigneusement lissée. Cette technique peut se retrouver sur l'une des faces de récipients peints sur l'autre face en "gris-sur-gris" (cf. infra § 3.2.), et il existe un certain nombre de points communs entre ces deux techniques.

3.1.2.3. Évolution chronologique. Contrairement à l'idée parfois admise, cette catégorie est présente dès les niveaux les plus anciens de la culture de Sesklo, notamment à Otzaki, où son évolution y est parallèle à celle de la céramique peinte en brun-rouge sur blanc, et dans des proportions comparables (tabl. 3). Sur les sites du Sud et de l'Ouest de la Thessalie en revanche, comme à Tsangli ou Sesklo, elle parait, nous l'avons vu, limitée à la fin de la culture de Sesklo (WaCe et Thompson [1912], Theocharis [1981], KotSakis [1983]). Si cette situation était confirmée, elle suggérerait une antériorité de cette catégorie dans le Nord de la Thessalie; certains sites sud-occidentaux, comme Tsani, l'ignorent totalement. En l'état actuel des fouilles de Plateia Magoula, il s'agit dans les niveaux les plus profonds (tabl. 5), non seulement de l'unique catégorie à décor peint, mais de la catégorie céramique majoritaire. Elle représente en effet les deux-tiers du total à $-8,2 \mathrm{~m}$, ce qu'on ne peut mettre seulement au compte de différences spatiales ou fonctionnelles, puisque dans les niveaux légèrement supérieurs, cette céramique suit une courbe descendante régulière mais cohérente avec ces premières valeurs; elle reste abondante jusqu'à $7 \mathrm{~m}$, diminue ensuite fortement mais sans ruptures, pour disparaître vers $5 \mathrm{~m}$. Son déclin paraît lié à celui de la céramique fine non décorée, tout comme à l'augmentation régulière de la céramique grossière. Notons qu'il n'y a aucun point de la séquence de Otzaki qui correspondrait à la situation de Plateia Magoula entre 8,25 et $7 \mathrm{~m}$, où la céramique à décor frotté est dix à vingt fois plus importante que la céramique peinte en brun-rouge; il y a donc nécessairement un phénomène de variabilité régionale. Enfin, la céramique à décor frotté n'est présente ni à Makrychori, ni à Arapi.

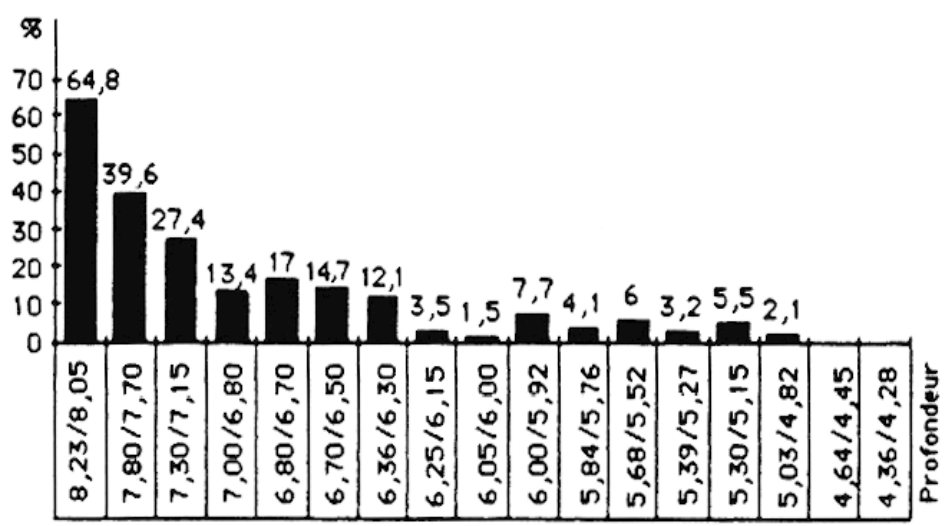

TABleau 5. - Evolution stratigraphique (en poids) de la céramique à décor frotté sur le site de Plateia Magoula Zarkou. 
3.2. La céramique gris-sur-gris (fig. $7-8,20,24)$.

\subsubsection{Définition, technologie et stratigraphie.}

La céramique gris-sur-gris ("grey-on-grey", "grau-auf-grau" ou $\Gamma 1 \beta$ de Wace et Thompson; parfois faussement interprétée par ces auteurs ([1912] : p. 17 et 105), dans leur catégorie $\Gamma 1 \alpha 2$, comme une variété de décor "raclé») est l'une des plus caractéristiques du Néolithique thessalien, et sans doute l'une des plus technologiquement élaborées du Néolithique européen. Très tôt identifiée, sa répartition géographique inégale et sa faible abondance n'ont permis que récemment de préciser sa position stratigraphique exacte. Son attribution chronologique à la phase Tsangli n'est encore que supposée dans la publication de la stratigraphie d'Arapi (Hauptmann et Milojčćć [1969], p. 47) et elle n'a pu être définitivement établie qu'avec la fouille de la nécropole à incinérations puis de l'habitat de Plateia Magoula Zarkou.

Bien que son exceptionnelle qualité technique suggère une nette rupture technologique inaugurant l'avènement de la culture de Dimini ancien, la stratigraphie de Plateia Magoula Zarkou permet en fait de définir deux catégories technologiques transitionnelles, et l'on devra donc distinguer:

a) une céramique "prolo-gris-sur-gris ", à paroi fine, dont l'extérieur orangé (Munsell 5 YR 7/2) est identique à la céramique orange monochrome (cf. infra $\$ 3.6$.) ou à décor frotté des phases Sesklo classiques, et qui peut d'ailleurs comporter ce décor frotté. Il s'agit en général de gobelets légèrements ouverts à parois hautes, normalement agrémentés d'une large anse verticale en ruban, forme classique de la céramique à décor frotté (supra § 3.1.2.2. et fig. 6, 8). En revanche, l'intérieur de ces récipients est d'une couleur grise franche, comparable à celle de la céramique gris-sur-gris. Cette catégorie est présente dès les plus anciens niveaux actuellement atteints à Plateia Magoula Zarkou (tabl. 6) et poursuit ensuite une évolution "gaussienne " classique, sans jamais dépasser $4 \%$ environ du total de la céramique; elle tend à disparaitre entre 5,60 et $5,15 \mathrm{~m}$, parallèlement d'ailleurs à la céramique monochrome orange et au moment de l'essor véritable de la céramique gris-sur-gris. L'existence d'une telle céramique a été signalée notamment à Otzaki, Nessonis, Tsapocha, Tsangli et Servia (Hauptuann et Mrlojčrić [1969], p. 47; Theocharis [1973], p. 79 et 1981, p. 122; RIDLEY et WARDLE [1979], p. 213 et 217).

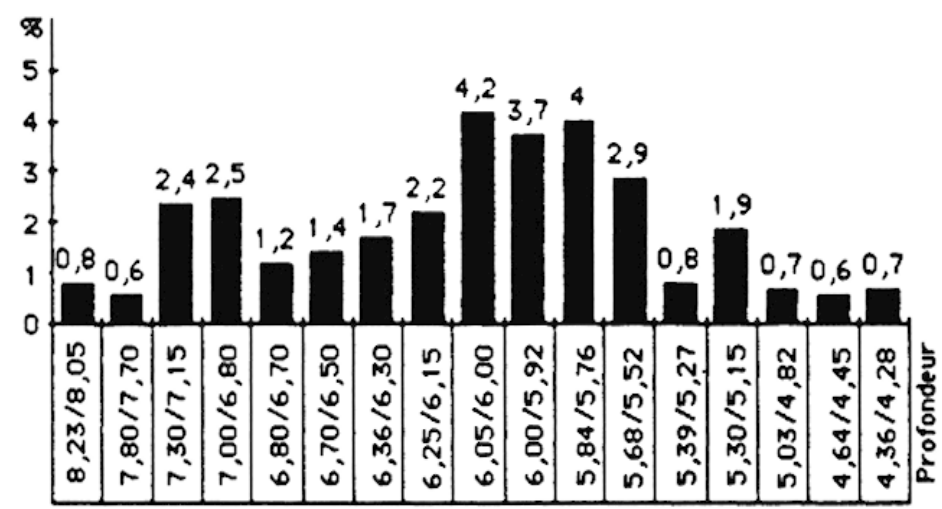

Tableau 6. - Evolution stratigraphique (en poids) de la céramique "proto-gris-sur-gris" sur le site de Plateia Magoula Zarkou. 

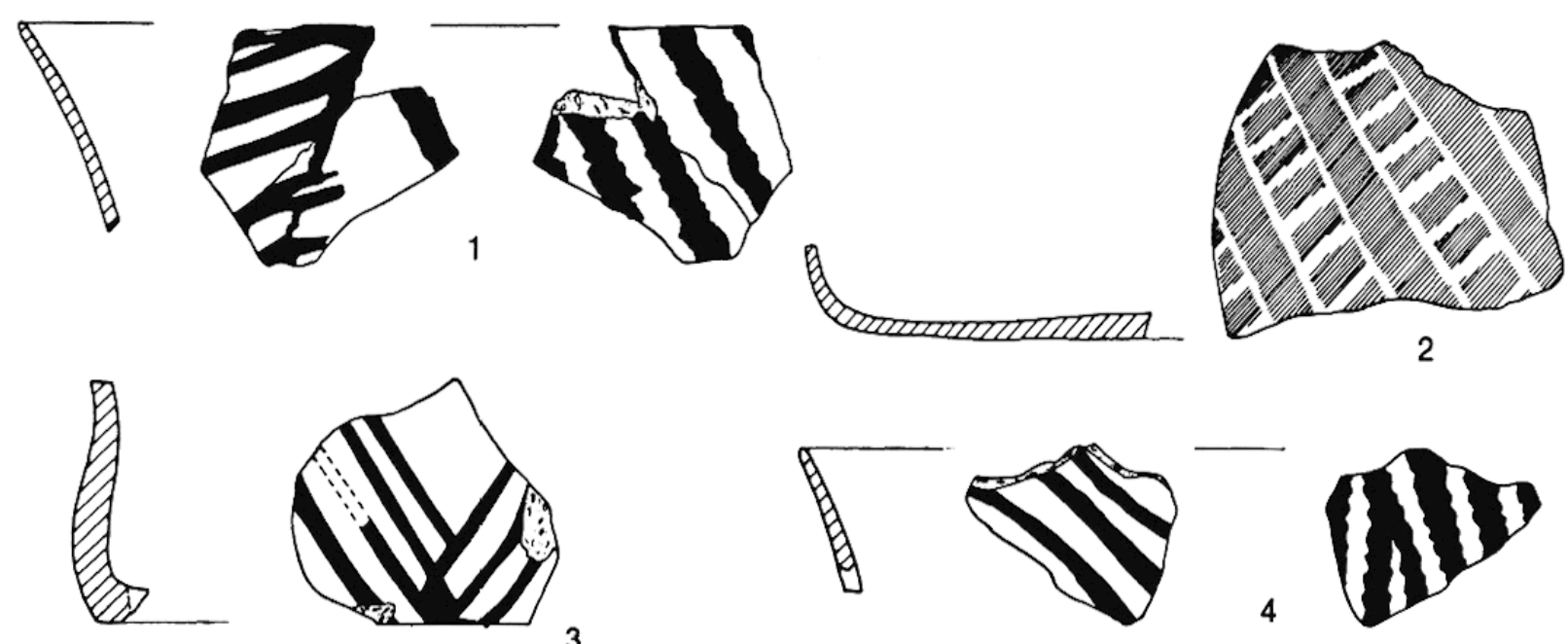

3
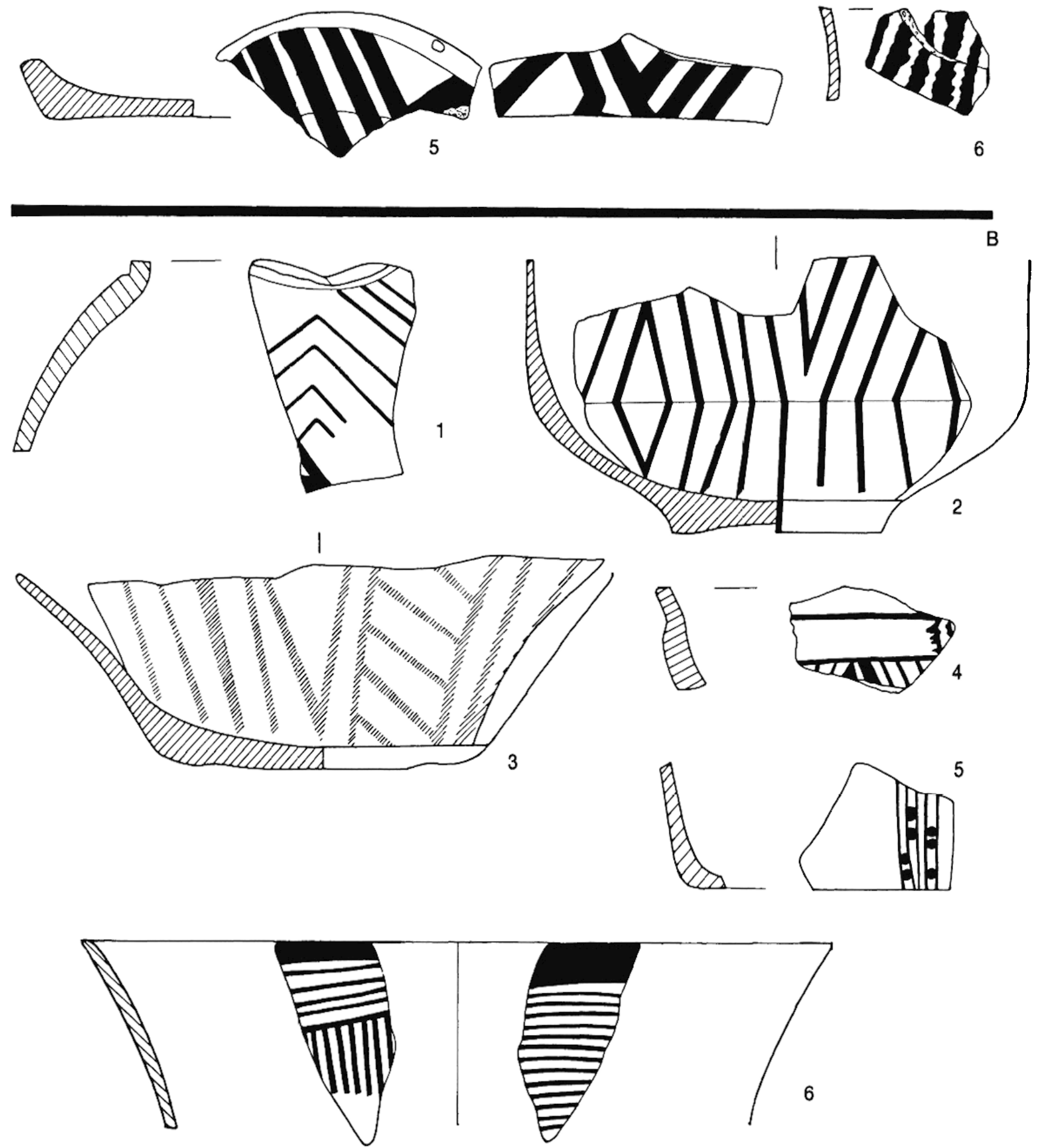

Fig. 7. - A) Céramique "proto-gris-sur-gris" (Plateia Magoula Zarkou); B) Céramique gris-sur-gris (Plateia Magoula Zarkou). Env. 1:3. 
b) une céramique gris noirâtre, de couleur homogène (proche de Munsell N4) sur les deux faces, comparable quant aux formes et aux décors à la céramique gris-sur-gris, mais qui n'atteint jamais le gris clair (Munsell N7) soutenu de cette dernière; la pâte est souvent moins fine. Ces teintes grisnoirâtre sont d'ailleurs imparfaitement standardisées à l'intérieur de la catégorie. L'évolution stratigraphique à Plateia Magoula Zarkou (tabl. 7) se traduit par une courbe également "gaussienne", mais en dents de scie, et sensiblement parallèle à celle de la céramique "proto-grissur-gris". Il est donc tentant de considérer cette catégorie mal standardisée comme une variante intermédiaire, où la teinte grise franche est le but recherché mais pas toujours atteint. Certains fragments se trouvent d'ailleurs à la limite des deux catégories.

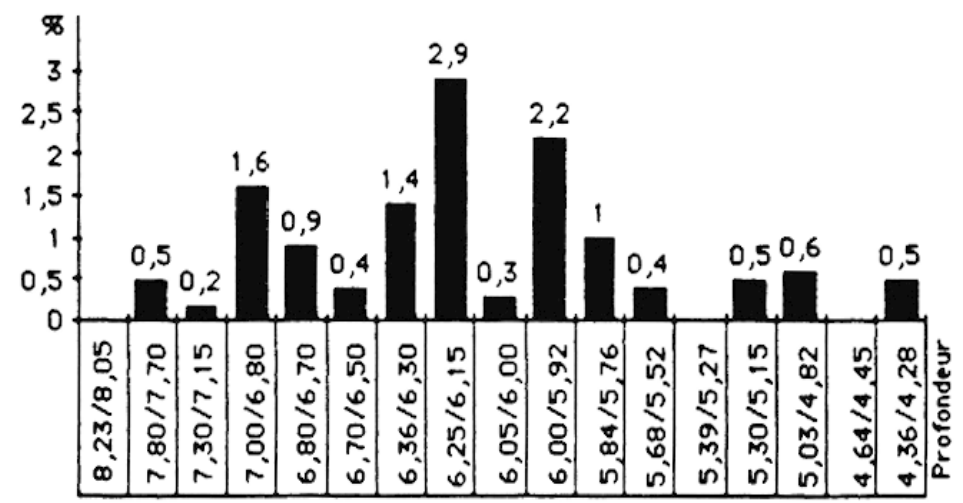

TABreau 7. - Évolution stratigraphique (en poids) de la céramique gris-noirâtre sur le site de Plateia Magoula Zarkou.

c) la céramique gris-sur-gris proprement dite est d'un gris clair homogène (Munsell N7 ou légèrement plus clair) et apparaît comme très standardisée, comme l'ont montré les analyses physico-chimiques en cours (à paraître dans la seconde partie de cette étude). La pâte a fait l'objet d'un processus de "fusion" ("Versinterung", d'après LeTsch et Noll [1983], p. 121), exceptionnel dans le Néolithique européen et qui suppose une cuisson d'environ 1.000 degrés, contre 850 degrés pour toutes les autres catégories du Néolithique thessalien - la céramique protosesklo supposant seule une cuisson plus basse encore, aux alentours de 700 degrés. Cette céramique "sonne" donc comme de la porcelaine et elle n'a, la céramique "minyenne" mise à part, pratiquement pas d'équivalent parmi la poterie protohistorique de l'Ancien Monde. Les parois sont très fines, souvent inférieures à 4-5 mm. On distingue deux catégories de pâtes, l'une fine à dégraissant sableux, l'autre très fine et non dégraissée. La présence d'un décor peint en gris-noir justifie l'appellation. Cette production suppose une particulière maîtrise des problèmes technologiques, l'obtention en cuisson de "l'équilibre de Boudouard" et son mode de fabrication reste encore partiellement hypothétique (LETSCH et NoLL [1963], p. 141), et en particulier les conditions de conservation à très haute température d'un décor apparemment appliqué avec un crayon charbonneux, qui peut d'ailleurs varier de teinte. Comme on l'a vu (tabl. 8), si des tessons sont épisodiquement présents à Plateia Magoula dès $-7 \mathrm{~m}$, cette céramique ne se développe vraiment qu'à partir de $-5,3 \mathrm{~m}$, et surtout $5 \mathrm{~m}$; elle atteint en poids $7 \%$ de la céramique dans les niveaux les plus récents, ce qui, vu sa finesse, signifie une proportion plus importante encore en nombre de récipients. Dans les fouilles anciennes, notamment à Tsani et Tsangli, il avait déjà été relevé que cette céramique apparaît en fait dès les niveaux récents de la culture de Sesklo, nettement avant le début de Dimini ancien proprement dit (WACE et Thompson [1912], p. 102 et 142, tabl. 63 et 90). Un autre fait de distribution est l'abondance de cette céramique dans les fouilles et les ramassages de surface de 

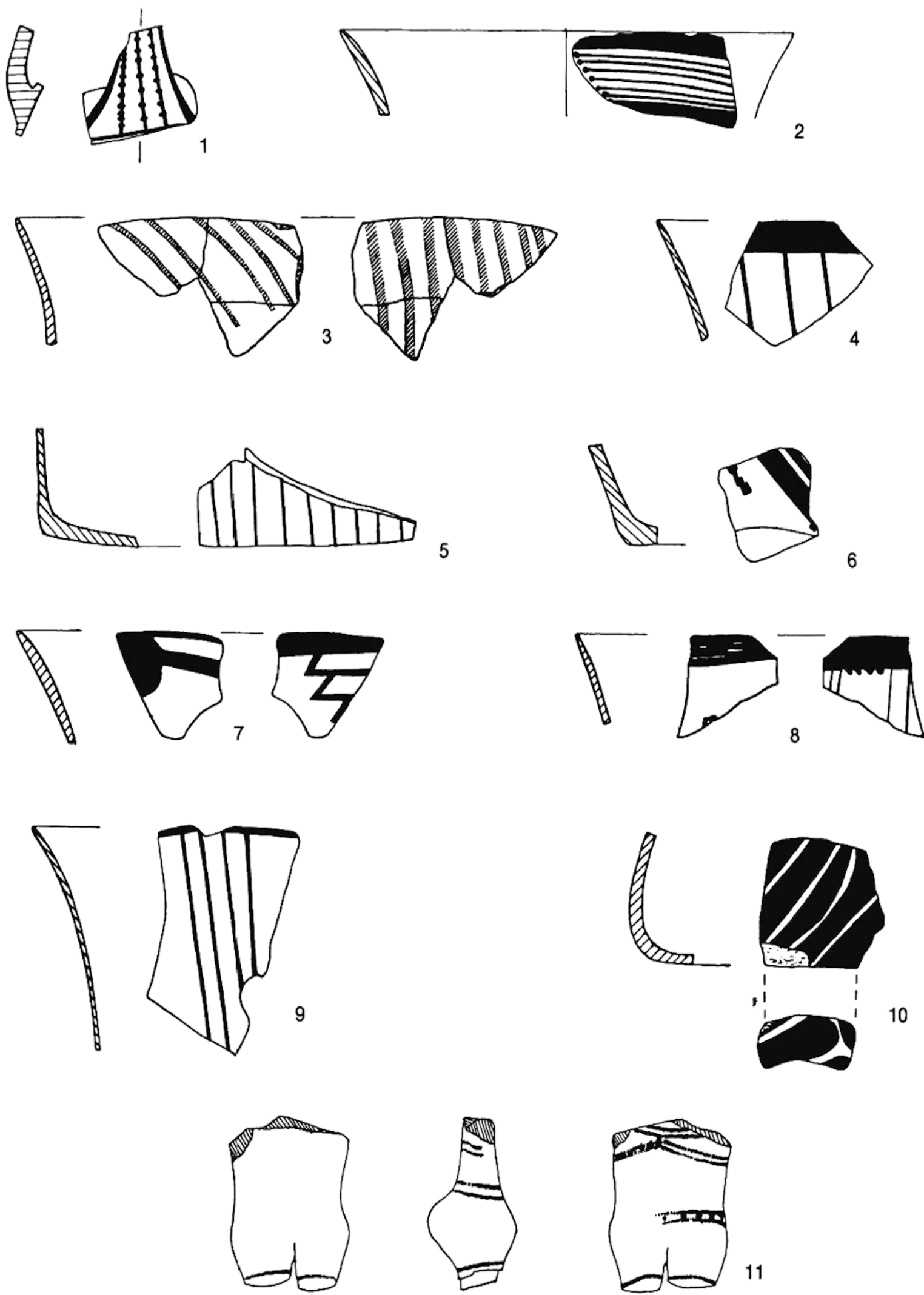

11
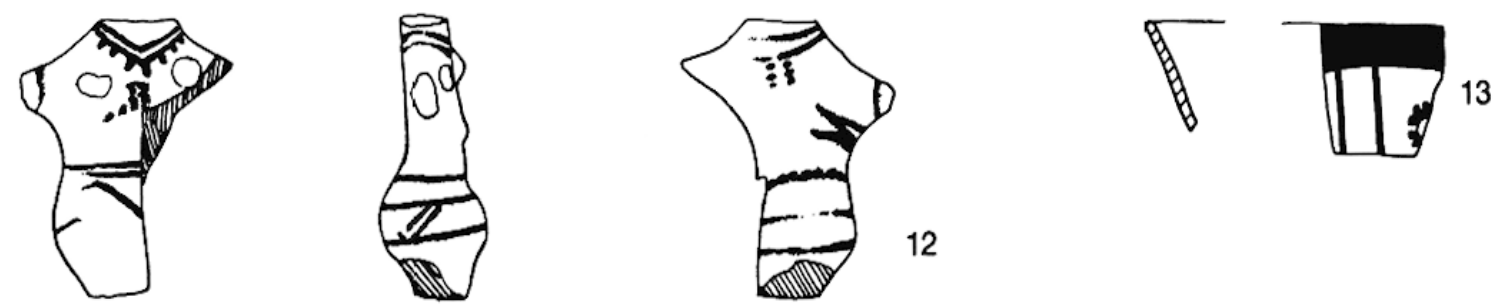

Fig. 8. - Céramique gris-sur-gris. $\mathrm{V}^{\mathrm{m}}$ 1-10 : Plateia Magoula Zarkou; 11 : Nikaia (surface); 12 : Petrino (surface); 13 : Platykampos (surface). Env. 1:3. 
Thessalie occidentale, qui contraste avec sa rareté sur les sites de Thessalie orientale, Plateia Magoula occupant à cet égard une position intermédiaire. C'est ainsi qu'à Makrychori 2, où elle est donc peu fréquente, elle est présente dès le début et disparaît vers $2,3 \mathrm{~m}$, après une diminution régulière (tabl. 9).

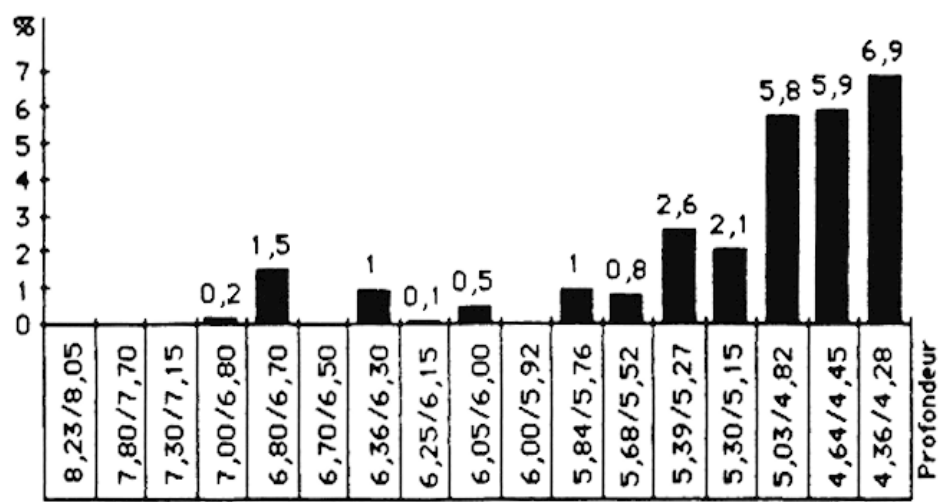

TABiEaU 8. - Évolution stratigraphique (en poids) de la céramique gris-sur-gris sur le site de Plateia Magoula Zarkou.

\begin{tabular}{|c|c|c|c|}
\hline & \multicolumn{3}{|c|}{ Niveau } \\
\hline & $3,8 / 3,4$ & $3 / 2,75$ & $2,45 / 2,25$ \\
\hline Cér. noire lissée & 6,7 & 7,5 & 11,3 \\
\hline Cér. peinte noire sur rouge $\ldots \ldots \ldots \ldots \ldots$ & 0 & 0 & 0,05 \\
\hline Cér. peinte mate brun sur brun $\ldots .$. & 0,9 & 0,3 & 0,1 \\
\hline Cér. polychrome Arapi $\ldots . \ldots \ldots \ldots \ldots \ldots$ & 0,3 & 0,1 & 0,3 \\
\hline Cér. peinte brun sur engobe blanc $\ldots \ldots \ldots$. & 0,1 & 0 & 0 \\
\hline Cér. gris-sur-gris $\ldots \ldots \ldots \ldots \ldots \ldots$ & 0,1 & 0 & 0,1 \\
\hline Cér. peinte en rouge $\ldots \ldots \ldots \ldots \ldots \ldots \ldots$ & 0,3 & 0 & 1 \\
\hline Cér. monochrome cuir lissée & 0,3 & 1,2 & 0 \\
\hline Cér. monochrome rouge $\ldots \ldots \ldots \ldots \ldots$ & 0 & 1,5 & 3,7 \\
\hline Cér. monochrome brun clair & 0 & 6,7 & 0,9 \\
\hline Cér. monochrome brun noir $\ldots \ldots \ldots \ldots \ldots$ & 0 & 0 & 2,1 \\
\hline Cér. grossière $\ldots \ldots \ldots \ldots \ldots \ldots \ldots \ldots$ & 91,3 & 82,8 & 80,5 \\
\hline
\end{tabular}

Tableau 9. - Pourcentage des différentes catégories céramiques dans un échantillon de trois couches des niveaux "Tsangli-Larissa" du site de Makrychori (décomptes en poids).

L'existence de ces trois catégories contiguës suggère donc l'hypothèse d'expérimentations et d'enchaînements technologiques successifs et continus, qui se stabilisent à des moments donnés sur certains types plus standardisés. L'utilisation d'une cuisson réductrice susceptible d'obtenir la couleur grise est également cohérente avec le développement contemporain d'une céramique noire lissée dite de "Larissa" (infra §3.4.); la cuisson réductrice, attestée au tout début du Néolithique ancien (périodes dites "Frühkeramikum" puis "protosesklo") par une céramique fine monochrome noire bien lissée, s'était en effet effacée par la suite. Un autre argument de proximité concerne, on le 
verra, le répertoire de motifs, très proche, surtout dans les phases anciennes de la céramique gris-sur-gris, de celui de la céramique à décor frotté, tout comme d'ailleurs les formes céramiques, et en particulier les gobelets hauts à anse en ruban. La filiation possible entre ces deux catégories avait d'ailleurs été relevée naguère par $D$. Theocharis, qui notait sur les sites de Tsangli, Servia et Tsapocha Magoula la présence, dans les niveaux de transition entre Sesklo et Dimini ancien, de tessons, appelés ici "proto-grissur-gris", combinant des caractéristiques des deux périodes, c'est-à-dire à surface rougeâtre et décor frotté à l'extérieur, et gris à l'intérieur (TheochaRis [1973], p. 79 et [1981], p. 122).

\subsubsection{Formes ET DÉCORS.}

Les formes de la céramique gris-sur-gris sont relativement variées. La forme dominante est une écuelle évasée à parois hautes, de dimensions diverses, et dont le profil évoque pour les exemplaires de grande taille, mais en plus abrupt, les écuelles de la céramique Sesklo peinte en brun sur blanc, et pour ceux de petite taille, en moins sinueux, les gobelets à parois hautes et à large anse verticale en ruban de la céramique à décor frotté (fig. $15, \mathrm{n}^{\text {os }} 12,43$ ). Mais il existe également des écuelles à épaulement marqué (fig. 15, $\mathrm{n}^{\circ} 65$ ), des bouteilles à anse (fig. 15, $\mathrm{n}^{\mathrm{os}} 50,57$ ) et des vases à piédestal ; ces formes en revanche se retrouvent dans les catégories peintes en noir sur rouge et en brun sur brun de Dimini ancien (infra $\S 3.3 .1 .1$. et 3.3.1.2.). Le dépôt de céramique gris-sur-gris dans la nécropole de Plateia Magoula Zarkou (tabl. 10 et 29), où elle représente $60 \%$ de la céramique funéraire, montre qu'il s'agit de récipients valorisés, ce que confirme l'importance statistiquement plus grande de trous de réparation, phénomène plusieurs fois noté (W ACE et Thompson [1912] p. 17, 111 ; Gallis [1982] p. 104, 108-109). Ce fait est également confirmé par l'utilisation de cette même technique pour la réalisation de figurines anthropomorphes (fig. 8, n*s $11-12$ et 24). Une évolution chronologique n'est perceptible que pour les gobelets hauts, dont les formes à partie inférieure saillante et bombée (fig. 8, $\mathrm{n}^{\circ} 10$ ) sont limitées aux niveaux les plus anciens, et font place ensuite à un profil concave net (fig. 8, $\mathrm{n}^{\mathrm{os}} 5-6$ ).

\begin{tabular}{|l|c|c|c|c|c|}
\hline & $\begin{array}{c}\text { Céramique } \\
\text { Grossière }\end{array}$ & $\begin{array}{c}\text { Céramique } \\
\text { Monochrome }\end{array}$ & $\begin{array}{c}\text { Céramique } \\
\text { Gris-Sur-Gris }\end{array}$ & $\begin{array}{c}\text { Céramique } \\
\text { Larissa }\end{array}$ & $\begin{array}{c}\text { Céramique } \\
\text { Peinte }\end{array}$ \\
\hline Nécropole ...... & - & $30 \%$ & $60 \%$ & $10 \%$ & $?$ \\
\hline Habitat récent ... & $61,1 \%$ & $18,4 \%$ & $7,2 \%$ & $1,5 \%$ & $11,7 \%$ \\
\hline $\begin{array}{l}\text { Habitat récent } \\
\text { (sans grosière) ... }\end{array}$ & & & & $3,9 \%$ & $30,1 \%$ \\
\hline
\end{tabular}

Tableau 10. - Pourcentages comparés des différentes catégories céramiques dans l'habitat (niveaux récents supérieurs à $-5 \mathrm{~m}$ ) et dans les tombes à incinérations de la nécropole de Plateia Magoula Zarkou.

Les décors sont normalement peints en gris-noir. Le pigment a le plus souvent un aspect mat, voire érodé, et il évoque alors l'aspect de certains décors frottés non lissés; mais il peut également être lissé. On rencontre également une pigmentation rouge, orangée, brune, voire rosâtre, qui pourrait tenir à des altérations dues à la cuisson - 
comme on les rencontre dans d'autres cultures avec d'autres pigments sombres, notamment le pigment noir au manganèse de la céramique thessalienne noire sur rouge des périodes Dimini-Tsangli puis Dimini-Otzaki, ou le pigment au graphite de la céramique macédonienne du Néolithique récent et du Chalcolithique. Les thèmes décoratifs des niveaux les plus anciens sont comparables à ceux de la céramique à décor frotté (avec parfois, on l'a vu, co-existence des deux techniques sur un même récipient), voire de la céramique $A 3 \beta$. Il s'agit de chevrons composés de plusieurs lignes parallèles, les deux lignes externes pouvant être beaucoup plus épaisses, ou de motifs en "arêtes de poisson" (fig. 7-8). Dans les niveaux plus récents, le répertoire s'enrichit considérablement, et entretient cette fois des rapports certains avec celui de la céramique peinte mate en brun sur brun $(\$ 3.3 .1$.2. et fig. $9 \mathrm{~B})$ : U opposés, zigzags verticaux arrondis, lignes de points, décor décalé, petits motifs variès de remplissage, etc. (fig. 7-8, 20).

\subsection{Les styles peints de type Tsangli.}

La mise en place de la culture de Dimini ancien s'accompagne d'un profond renouvellement à la fois des technologies du décor et des styles eux-mêmes. L'intérêt de la stratigraphie de Plateia Magoula Zarkou est de montrer qu'il ne s'agit pas d'une mise en place soudaine, voire importée de toute pièce; mais que la période de transition que l'on peut distinguer entre $-6 \mathrm{~m}$ et $-5 \mathrm{~m}$ environ est en particulier une période d'expérimentation, où des recherches techniques sont menées, en continuité avec les savoirs antérieurs, sans toujours aboutir d'ailleurs à des types stables. Il est de ce fait difficile d'établir une typologie précise et fermée de ces tentatives, d'autant que se présenteront aussi bien des expérimentations sans suite, voire de simples accidents de cuisson, que des types stables mais rares et brefs, ou enfin de véritables "fossiles directeurs". Il est évident que des fouilles minutieuses portant sur cette phase risqueront d'accroître encore l'éventail des techniques identifiables. On procèdera donc ici à des regroupements sommaires :

\subsubsection{Les styles à décor peint en un SEul ton (fig. 9-10, 21).}

3.3.1.1. La céramique à décor peint en noir sur rouge (fig. $9 \mathrm{~A}$ ). Cette technique utilise un pigment au manganèse et une cuisson oxydante (LETSCH et Noll [1983], p. 131-134). Identifiée par WACE et Tном PSON ([1912] : "B3d»), qui la distinguent, par son aspect mat et son style linéaire, du décor noir sur rouge qui deviendra typique de Dimini-Otzaki ("B3a2", seconde variante), elle n'apparaît en Thessalie qu'avec Dimini ancien, alors qu'elle est connue au moins dès le milieu du "Néolithique ancien" des cultures balkaniques plus septentrionales (par exemple dans les groupes de Starčevo, AnzabegovoVršnik, Veluška Tumba-Porodin, Cavdar, Gradešnica, etc). On la retrouvera ensuite continuement, avec des motifs différents, aux périodes Arapi et Otzaki. Au temps des phases Dimini récentes, elle est présente, avec une technique de cuisson bien supérieure, en Macédoine centrale et orientale, dans les groupes d'Olynthe-Vassilika et de Dikili Tach; un style macédonien tardif spécifique, dit "de Galepsos", situé à l'orée du Chalcolithique ancien (Sitagroi III-Gumelnitsa-Karanovo VI) est attesté sous forme d'importations en Thessalie sur le site maritime de Pefkakia.

Il s'agit d'une technique très caractéristique, même s'il peut exister quelques exemplaires intermédiaires entre ce style et la peinture mate brun-sur-brun (infra $\S 3.3 .1 .2)$ qui en est proche mais s'en distingue usuellement; ces cas intermédiaires 

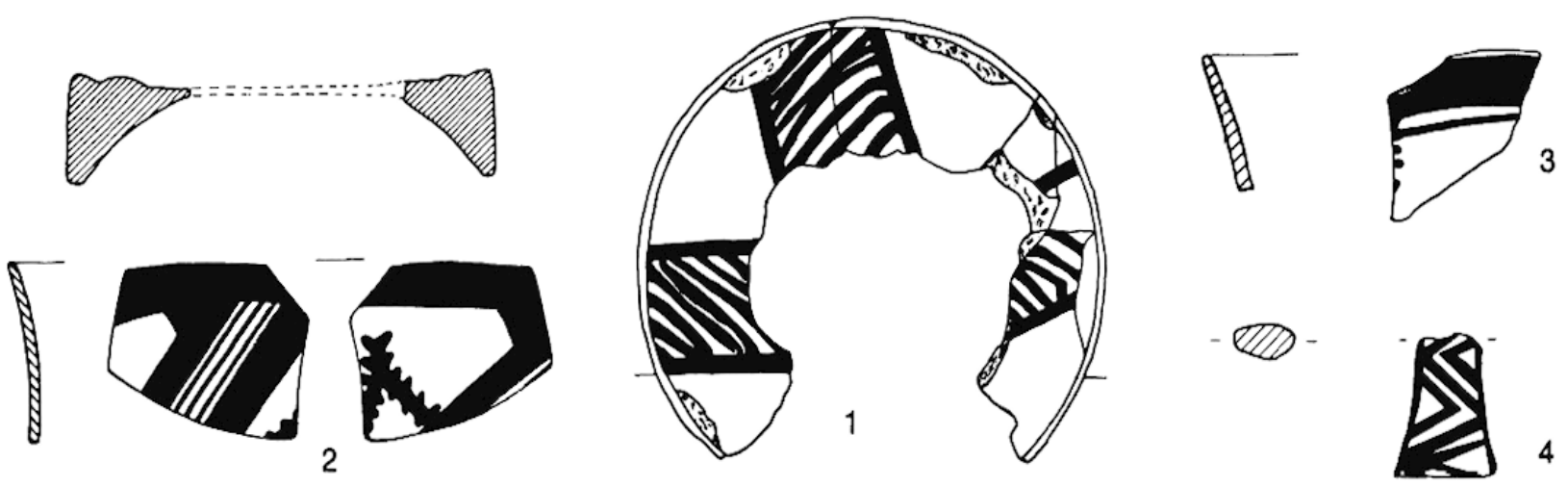

-
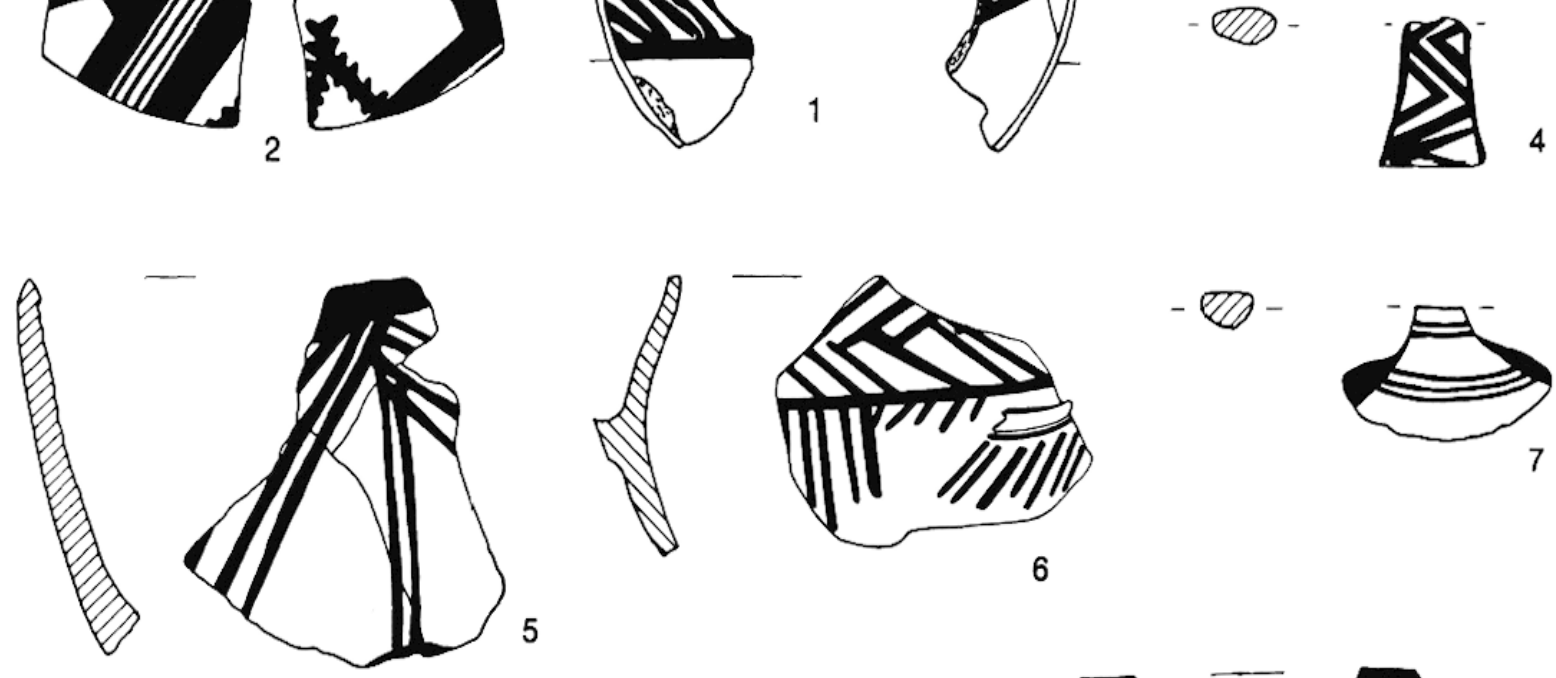

-
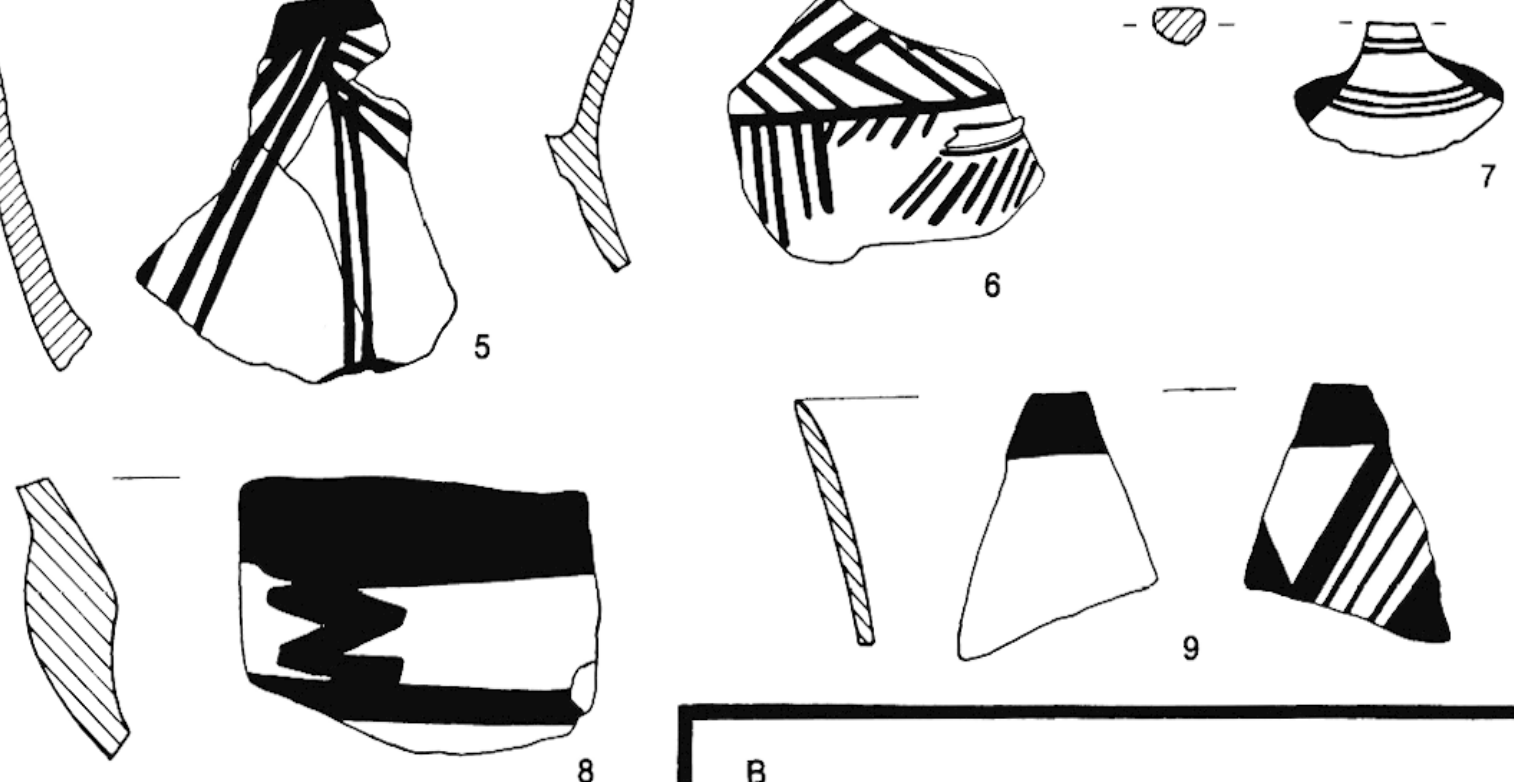

B
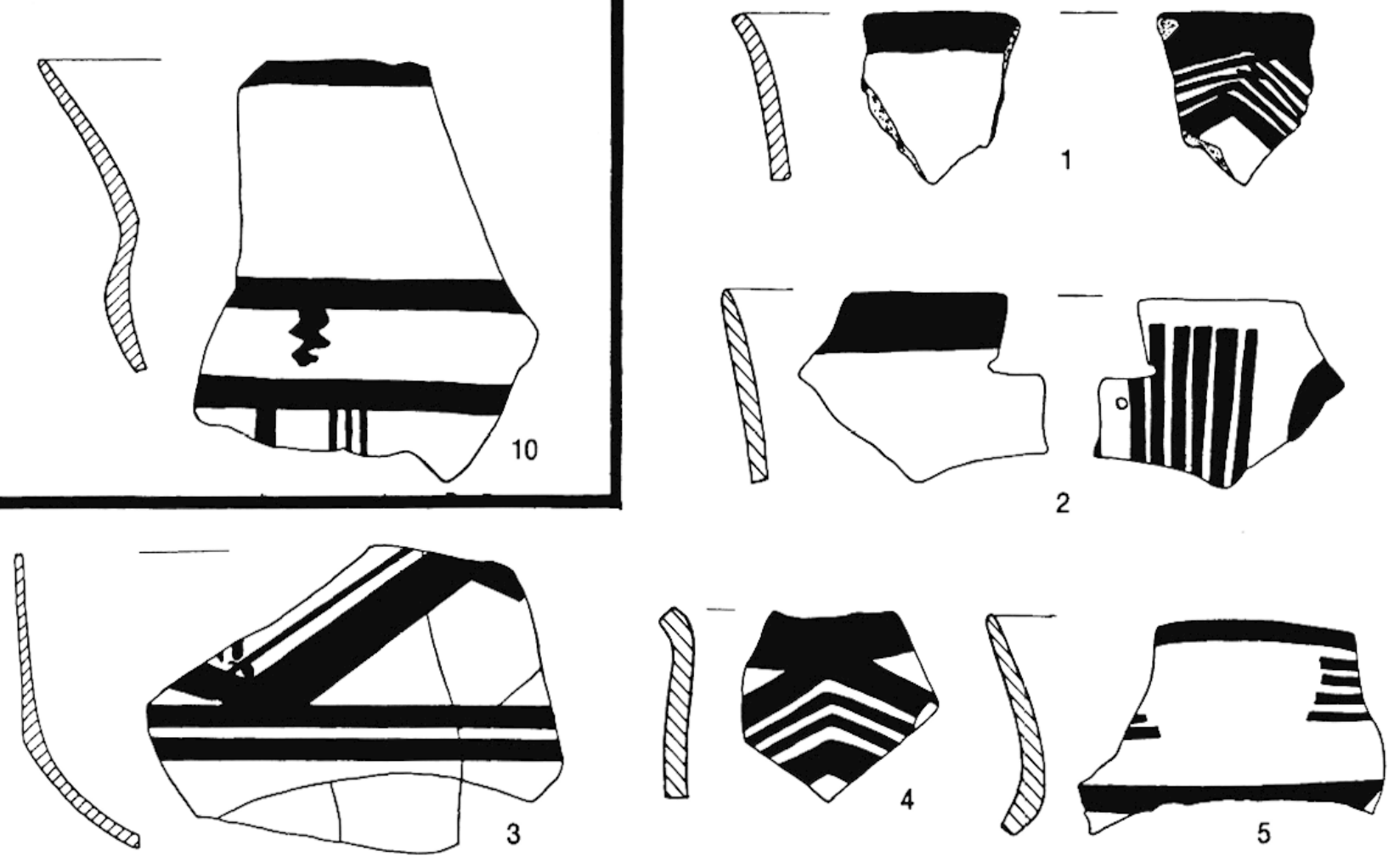

Fig. 9. - A) Céramique peinte en noir sur rouge. 1-9 : Plateia Magoula Zarkou; 10 : Tsalmas. B) Céramique peinte mate en brun-sur-brun (Plateia Magoula Zarkou). Env. 1:3. 


\begin{tabular}{|c|c|c|c|c|c|c|c|}
\hline & $\begin{array}{l}\text { écuelles } \\
\text { tronconiques }\end{array}$ & $\begin{array}{l}\text { écuelles } \\
\text { carénées } \\
\text { bitronconiques }\end{array}$ & $\begin{array}{l}\text { écuelles } \\
\text { évasées à } \\
\text { épaulement }\end{array}$ & $\begin{array}{l}\text { voses à } \\
\text { piédestal }\end{array}$ & $\begin{array}{l}\text { gobelets } \\
\text { prolonds }\end{array}$ & bouteilles & $\begin{array}{l}\text { voses ouverts } \\
\text { hauts }\end{array}$ \\
\hline $\begin{array}{l}\text { peinture } \\
\text { brun-roug } \\
\text { sur clair }\end{array}$ & \&些 & & & & & & \\
\hline décor "frotté" & & & & & & & \\
\hline $\begin{array}{l}\text { céramique } \\
\text { gris-sur-gri }\end{array}$ & & & & & & & \\
\hline $\begin{array}{l}\text { céramique } \\
\text { Laríssa }\end{array}$ & & & & & & & \\
\hline$\underset{\text { mate }}{\text { peinture }}$ & & & & & & & \\
\hline $\begin{array}{l}\text { céramique } \\
\text { monochrome } \\
\text { demi-fine }\end{array}$ & & & & & & & \\
\hline $\begin{array}{l}\text { céramique } \\
\text { grossière }\end{array}$ & & & & & & ? & \\
\hline
\end{tabular}

TABleau 11. - Inventaire des principaux types de formes pour les différentes catégories de céramique. 
témoignent là encore de ces tâtonnements technologiques. Par suite d'accidents de cuisson, le pigment au manganèse peut prendre une teinte grisâtre ou blanchâtre. Cette technique peut apparaître également en polychromie, les lignes noires étant renforcées de blanc (infra $\S 3.3 .2 .3$ ).

Les formes caractéristiques sont une coupe à bord large évasé et épaulement très marqué, et une forme à piédestal ("compotier", "Fruchtständer»), dont la partie supérieure peut également adopter le profil précédemment décrit. Ces formes sont communes à d'autres techniques (tabl. 11). Elles ont parfois été attribuées par erreur à la culture de Rachmani (Milojčıć [1959], fig. 20, 1).

Le décor comprend deux thématiques principales, qui se retrouvent sur la céramique en brun-sur-brun et, avec moins de variété, sur la céramique en gris-sur-gris. Il s'agit d'une part de chevrons réalisés en plusieurs lignes parallèles, les deux lignes extérieures pouvant être plus épaisses, et courant tout autour du vase dans la tradition des décors Sesklo; d'autre part d'éléments verticaux, lignes parallèles, zigzags arrondis ou combinaisons plus complexes, qui peuvent comporter de petits motifs intermédiaires de remplissage. Ces éléments verticaux tendent donc à sectionner la surface au moyen de motifs isolés les uns des autres et moins couvrants, au contraire des thèmes précédents qui, dans la tradition Sesklo, entourent le récipient d'un motif continu ("Umlaufstil»). Ce sectionnement de la surface à décorer semble d'ailleurs cohérent avec l'architecture nouvelle des récipients, que les càrènes ou épaulements découpent en surfaces hétérogènes. Malgré la faiblesse des effectifs, il semble que la seconde thématique (éléments verticaux) se développe dans le temps au détriment de la première (chevrons continus). On trouvera également des répertoires de ces décors dans HauptmanN et Milojčić (1969) pl. 2, et Hauptmann (1981) pl. 7.

Stratigraphiquement, la peinture noire sur rouge apparaît à Plateia Magoula Zarkou de manière très sporadique dès $6,7 \mathrm{~m}$, mais ne connaît un certain développement qu'à partir de $5,3 \mathrm{~m}$, pour prendre son essor après $5 \mathrm{~m}$ (tabl. 12). Toutefois dès $7 \mathrm{~m}$, mais

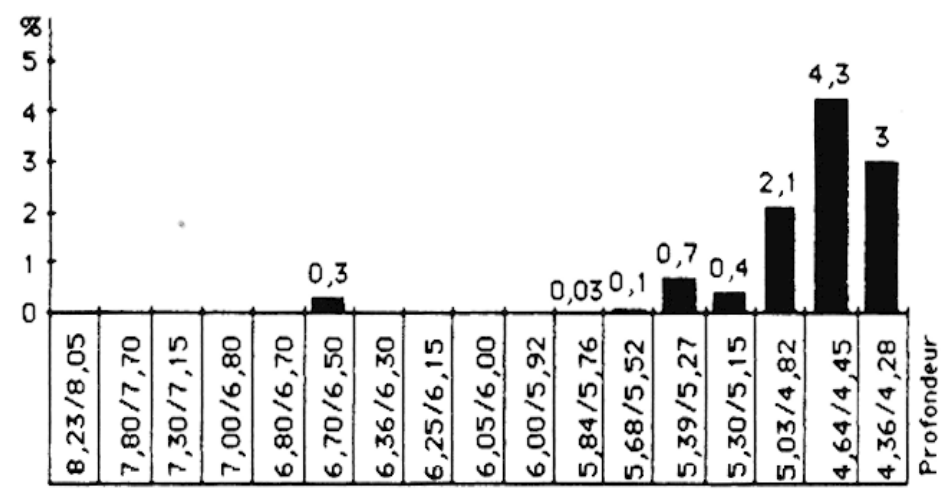

Tableav 12. - Evolution stratigraphique (en poids) de la céramique peinte en noir sur rouge, sur le site de Plateia Magoula Zarkou.

surtout dès $6 \mathrm{~m}$, un décor polychrome à dominante rouge utilise un pigment noir, souvent mal stabilisé, comme ornementation d'appoint (infra § 3.3.2.2). A Makrychori 2, présente dès le début mais jamais en quantité très abondante, la peinture noire sur rouge disparaît vers $2,2 \mathrm{~m}$, au moment de la transition vers la période Arapi, où une céramique noire sur rouge, avec une thématique différente et une surface très lustrée, est néanmoins attestée. 
3.3.1.2. La céramique peinle mate en brun-sur-brun. La céramique "matt-painted" est considérée comme typique de la phase Tsangli, et même de l'horizon qui marquerait partout en Grèce l'avènement, dans la terminologie locale, du "Néolithique récent" ("Late Neolithic", "Spätneolithikum»), un phénomène pour lequel D. Theocharis (1981), p. 128 a proposé le terme de "koinê". L'appellation "matt-painted" est cependant ambiguë, dans la mesure où des peintures "mates" se rencontrent à n'importe quelle période, où cet horizon comporte aussi des peintures "brillantes", notamment la peinture noire sur rouge le plus souvent, et où l'aspect "mat" peut tout aussi bien tenir à des accidents technologiques, voire post-dépositionnels. D'après des expérimentations récentes (LETSCH et Noll [1983], p. 134-135), cet aspect tiendrait à l'application de la peinture sur une surface déjà complètement sèche et devenue absorbante, donnant ainsi à la couche de peinture un aspect inégal et inhomogène, que la cuisson renforcera encore. On limitera donc le terme de "peinture mate" à cette seule peinture brun-sur-brun, attestée à cette époque à la fois dans les groupes culturels mal définis de Grèce centrale et

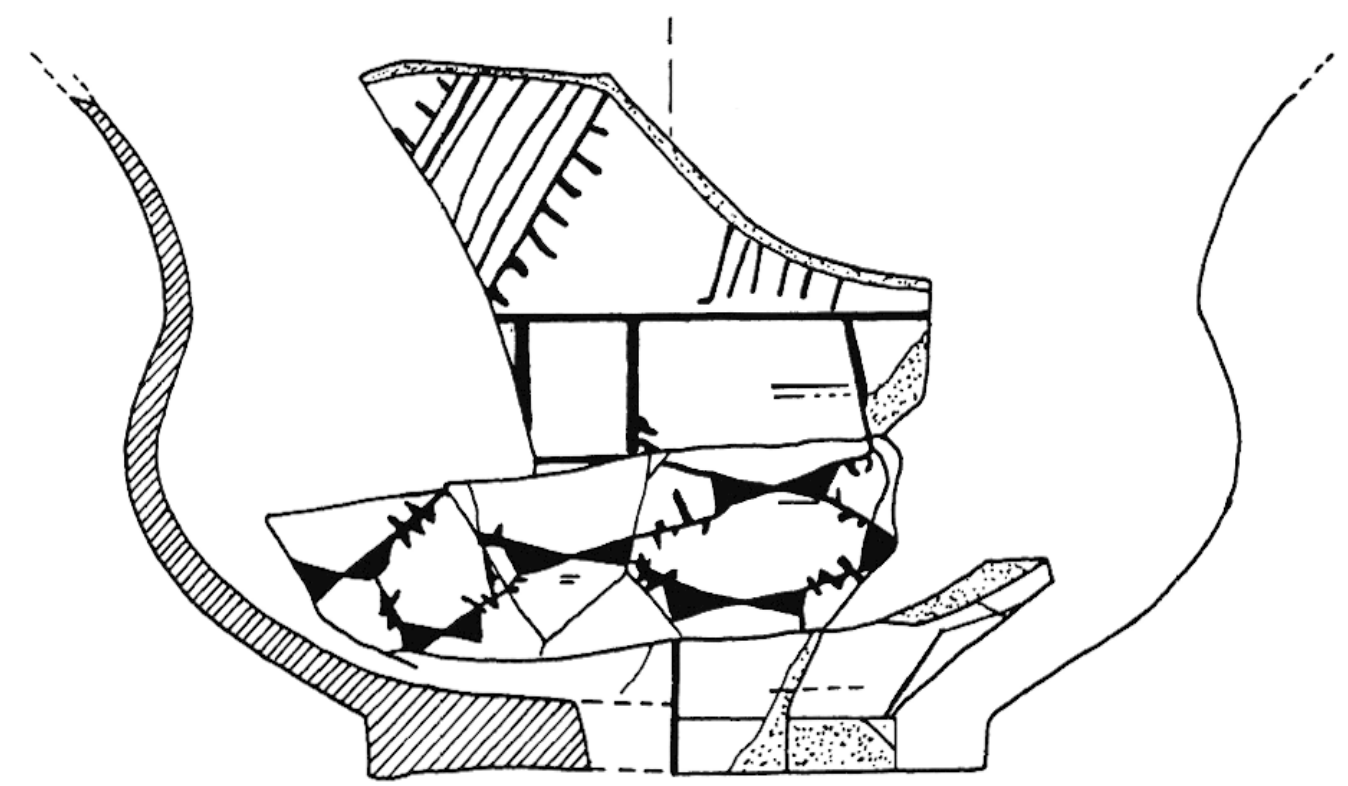

Fig. 10. - Forme et décor de transition entre Sesklo et Tsangli-Larissa (Magoula Aghiou Georghiou, Sofades, trouvaille de surface). Env. $1: 3$.

méridionale (Hauptmann et Milojčıć [1969], p. 27 ; Schachermeyr [1976]) et en Grèce septentrionale avec le "style d'Akropotamos" (Mylonas [1941]) et les groupes de Vassilika (Grammenos [1984], de Sitagroi I/II-Dikili Tach I, et de la vallée bulgare du Strymon (PEllerin [1986]).

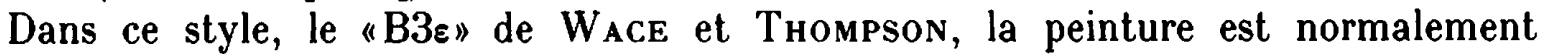
appliquée sans engobe (Hauptmann et Mrlojčıć [1969], p. 26-29). Ce dernier, quand il existe, est appliqué avec un pinceau laissant souvent des traces de stries visibles. La peinture elle-même peut parfois avoir un aspect brillant. Le pigment offre toutes les teintes du brun; la surface du récipient varie de brun clair à jaune pâle.

Parmi les formes, on peut identifier des gobelets ou écuelles tronconiques à parois concaves, proches de formes de la céramique gris-sur-gris, des écuelles carénées, des écuelles à épaulement marqué et large bord évasé, des vases à piédestal ("compotiers"), des cruches à col, toutes formes communes avec d'autres styles décoratifs (tabl. 11). 
Les motifs sont identiques à ceux décrits pour la céramique peinte en noir sur rouge (supra $\S 3.3 .1 .1$ ).

Stratigraphiquement, la céramique peinte brun-sur-brun suit la même évolution, quoiqu'en quantité bien moindre, que la céramique noire sur rouge. Elle apparaît très sporadiquement à Plateia Magoula Zarkou dès $6,7 \mathrm{~m}$, mais ne se développe vraiment qu'à partir de $5 \mathrm{~m}$. A Makrychori 2, elle est présente en faible quantité dans tous les niveaux Tsangli. Par ailleurs, la céramique brun-sur-brun est, par rapport à la céramique noir sur rouge, proportionnellement nettement plus importante à Makrychori 2 (tabl. 9), et nettement moins importante à Plateia Magoula Zarkou (tabl. 12-13). Il y aurait donc là aussi variabilité régionale.

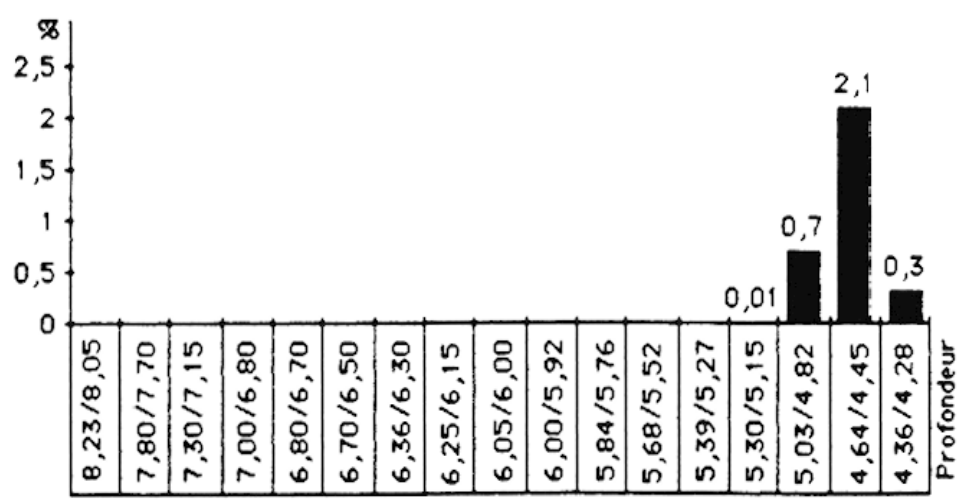

Tableau 13. - Evolution stratigraphique (en poids) de la céramique peinte mate en brun-sur-brun, sur le site de Plateia Magoula Zarkou.

3.3.1.3. La céramique peinte en rouge. A partir de $7 \mathrm{~m}$, mais surtout entre 6 et $5 \mathrm{~m}$, se développent en petite quantité à Plateia Magoula Zarkou des décors peints en rouge, de teintes diverses, sur des fonds, le plus souvent sans engobe, qui peuvent être indifféremment bruns sombres ou clairs, rougeâtres, voire noirâtres. Le caractère fragmenté et limité de l'échantillon, qui ne dépasse jamais $2 \%$ du total de la céramique, ne permet guère de définir clairement des motifs, le plus souvent composés de lignes parallèles, suggérant parfois des chevrons ou des "arêtes de poisson». La pâte est bien cuite et varie de fine à mi-fine. On peut identifier parmi les formes une coupe tronconique haute à parois évasées proche du type courant en gris-sur-gris et dans d'autres styles (tabl. 14), ainsi qu'un profi pansu de facture plus grossière.

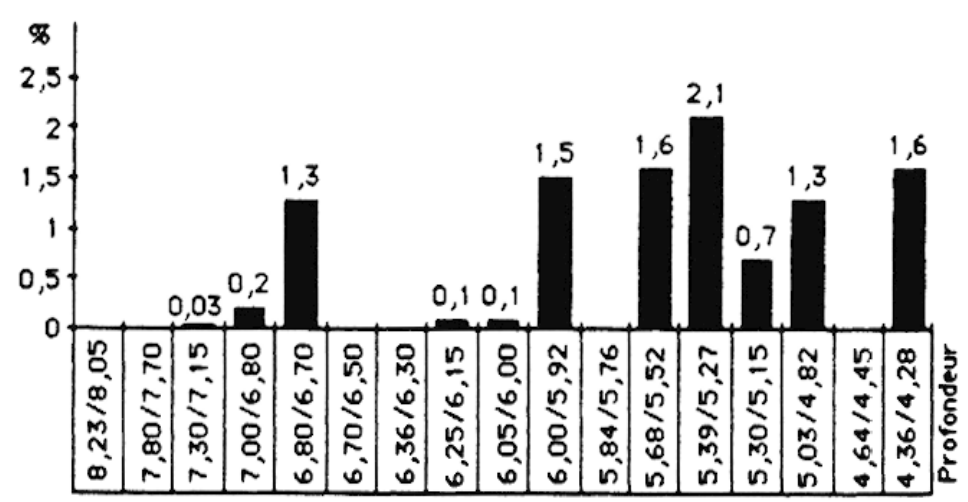

Tableau 14. - Evolution stratigraphique (en poids) de la céramique peinte en rouge sur le site de Plateia Magoula Zarkou. 
Par ses teintes et par son exécution plutôt négligée, ce type de décor pourrait être interprété comme une évolution à partir des éléments les plus tardifs de la peinture

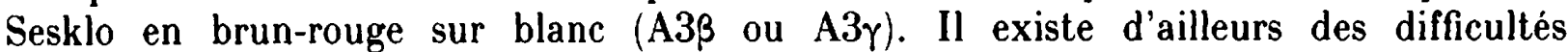
d'attribution entre ces deux styles pour certains fragments. De fait, présente dès $7 \mathrm{~m}$, cette céramique est surtout attestée entre 6 et $5 \mathrm{~m}$, au moment de la disparition définitive de la céramique peinte Sesklo, tandis qu'elle s'efface elle-même lors de l'essor des styles noir sur rouge et brun-sur-brun. La peinture rouge est également composante de styles polychromes (infra $\$ 3.3 .2 .1-2$ ).

3.3.1.4. La céramique à décor peint en brun sur engobe blanc (fig. $11 \mathrm{~A} ; 21, \mathrm{n}^{0} 2$ ). L'utilisation d'un engobe blanc, sans doute destiné à aviver les contrastes de couleur, est attestée en Thessalie au moins dès la période Sesklo, et elle se poursuit pendant tout le Néolithique, avec des hauts (Sesklo, Arapi, Dimini classique) et des bas (Tsangli-Larissa, Dimini-Otzaki). Cet engobe est à base de calcite. A Plateia Magoula Zarkou, on trouve de manière très sporadique et dès $7 \mathrm{~m}$ des fragments de récipients grossiers de grande taille comportant à la fois cet engobe et une peinture brunâtre, avec des motifs élémentaires linéaires (tabl. 15). L'engobe mat et crayeux contraste avec l'engobe brillant et soigné de la céramique Sesklo à peinture brun-rouge et évoque déjà l'engobe blanc caractéristique de la période Arapi. Des fragments qui ne comportent que cet engobe mais sans traces de peinture peuvent aussi appartenir à ce type. Un style polychrome particulier (infra \$3.3.2.1) peut également comporter un engobe comparable.

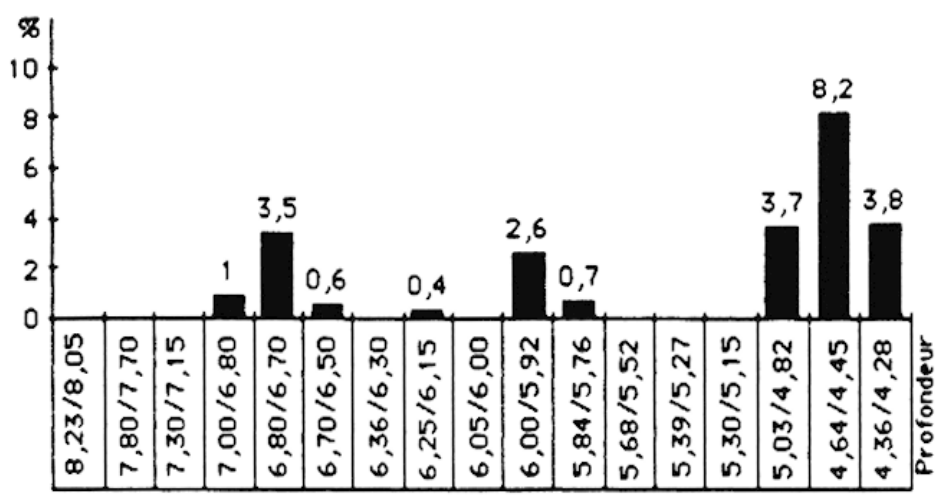

Tableau 15. - Évolution stratigraphique (en poids) de la céramique peinte en brun sur engobe blanc, sur le site de Plateia Magoula Zarkou.

\subsubsection{Céramiques à décors peints polychromes.}

3.3.2.1. Décor rouge cerné de noir sur fond clair ("B3z»). Ce décor, où le rôle principal est joué par le pigment rouge, est particulier à la Thessalie occidentale (W ACE et Thompson [1912], p. 17 et 101; Hauptmann et Milojčić [1969] p. 29; Theocharis [1973], p. 79 et [1981], p. 122), notamment sur les sites de Tsangli, Tsani et Tsapocha, mais apparaît parfois en Thessalie orientale, comme à Tsalmas (trouvailles de surface, Éphorie de Larissa). A Plateia Magoula Zarkou, géographiquement à la limite des deux zones, les trouvailles se situent surtout entre 5,6 et $5 \mathrm{~m}$, toujours en petit nombre. Diverses teintes sont attestées sur ces différents sites, le noir pouvant virer au gris, le fond pouvant être de couleur beige à rouge clair, avec ou sans engobe. Particulièrement caractéristique est l'existence d'un engobe blanc crayeux, analogue à celui de la catégorie 

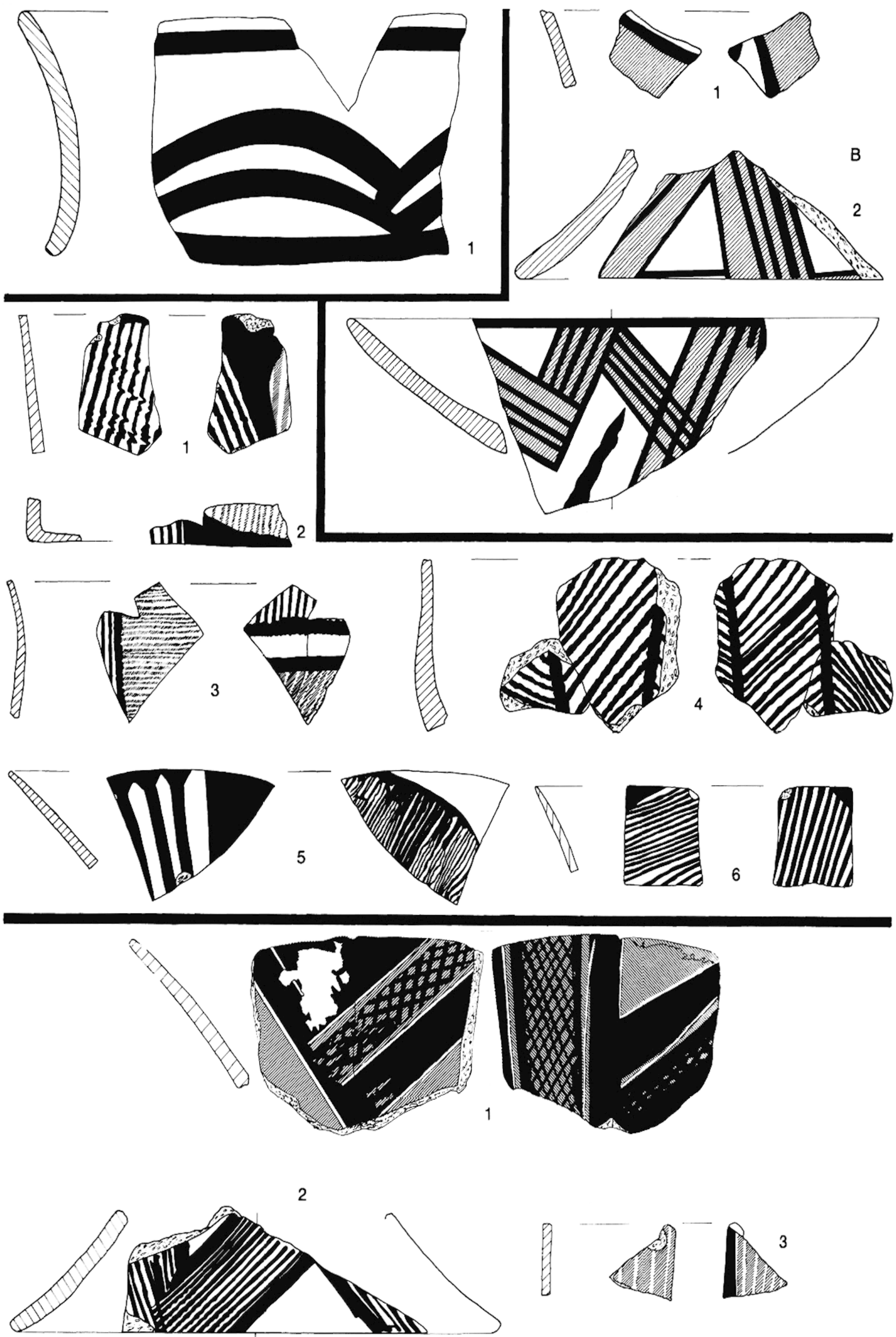

Fig. 11. - A) Céramique peinte en brun sur engobe blanc (Plateia Magoula Zarkou).

B) Céramique à décor polychrome peint en rouge cerné de noir (1: Plateia Magoula Zarkou; 2-3: Tsalmas).

C) Céramique polychrome peinte en rouge avec triangles noirs (Plateia Magoula Zarkou).

D) Céramique polychrome peinte en noir et blanc sur rouge (1-2: Tsalmas; 3: Plateia Magoula Zarkou). Env. 1:3. 
précédente. Les motifs sont faits de bandes larges d'un à deux $\mathrm{cm}$ et semblent surtout dessiner des chevrons. Les lignes noires délimitent ordinairement les rubans rouges, mais peuvent en outre les renforcer intérieurement (fig. $11 \mathrm{~B} ; 23, \mathrm{n}^{\circ} 2-3,5$ ). Les formes comportent au moins un "compotier" à pied largement évasé.

3.3.2.2. Décor peint en rouge sur clair, avec triangles de remplissage peints en noir (fig. $11 \mathrm{C} ; 22$ ). Ce style est peu représenté, mais très caractéristique. Présent à Plateia Magoula Zarkou dès $7 \mathrm{~m}$, il n'est bien attesté qu'entre 6 et $5 \mathrm{~m}$, sans jamais dépasser $2 \%$ du total de la céramique (tabl. 16). Il s'agit d'une céramique fine à mi-fine. Le décor apparaît en brun-clair, brun-rouge ou rouge, en fines lignes ondulées parallèles. L'ondulation résulte soit d'un mode d'application "tremblé", soit d'un lissage vigoureux sur une surface encore humide (fig. $11 \mathrm{C}, \mathrm{n}^{\circ} 1 ; 22, \mathrm{n}^{\circ} 7$ ). Ce dernier cas paraît suffisamment fréquent pour ne pas résulter d'une «maladresse" fortuite; il est obtenu par la même opération de lissage humide que certains décors "frottés" tremblés. Le pigment rouge lui-même paraît identique à celui de la céramique peinte en rouge (supra § 3.3.1.3). Ainsi cette catégorie se trouverait dans la continuité, à la fois de la céramique à décor frotté et de la céramique Sesklo peinte en brun-rouge. Mais le décor principal en rouge paraît bordé de triangles peints en noir, souvent pendant sous le bord, et qui parfois viennent empiéter sur le décor principal. Le pigment noir peut également apparaittre sous une forme décolorée grisâtre ou blanchâtre. Les fragments retrouvés ne permettent pas jusqu'à présent de reconstituer la thématique globale, mais on discerne les motifs de chevrons ou en "arêtes de poisson" connus avec d'autres styles. Les formes comprennent un gobelet tronconique haut à parois concaves, et une écuelle évasée à profil sinueux. Cette technique minoritaire est également attestée par des prospections de surface sur d'autres sites, notamment à Orphana et Keramidi (fig. 1).

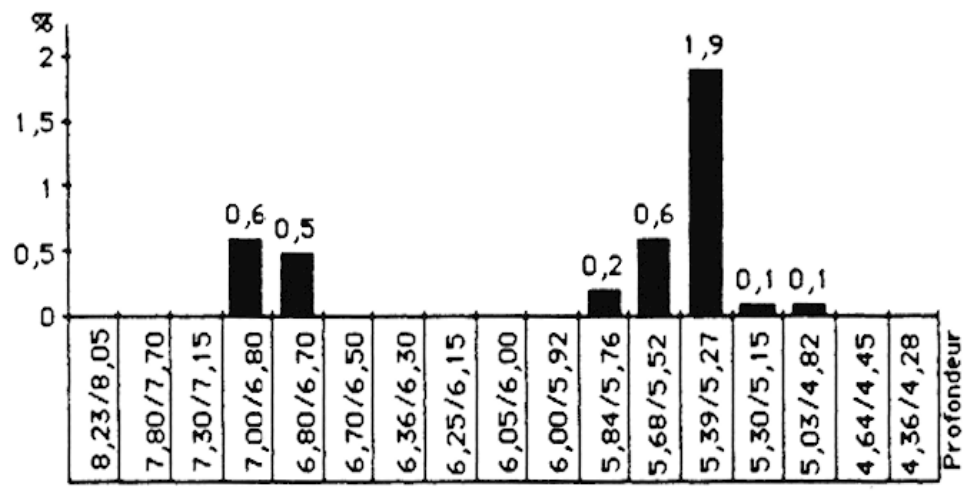

Tableau 16. - Évolution stratigraphique (en poids) à Plateia Magoula Zarkou de la céramique polychrome à décor rouge sur fond clair accompagné de triangles noirs.

3.3.2.3. Décors peints en noir et blanc sur fond rouge. De même que les deux précédents styles apparaissaient comme des variantes plus complexes de la céramique peinte en rouge, cet autre style paraît procéder de la céramique peinte en noir sur rouge (supra § 3.3.1.1), comme en témoigne le rôle principal du pigment noir et le fond rouge brillant. La couleur blanche n'existe dans la culture de Sesklo qu'avec le style A3a, ancien et rare, si l'on excepte l'aspect blanchâtre de certains décors frottés, et elle n'est présente sinon dans notre corpus qu'en décor également d'appoint et peu fréquent sur la céramique noire lissée (infra $\$ 3.4$ ); quelques tessons comportaient également entre 4,6 et 

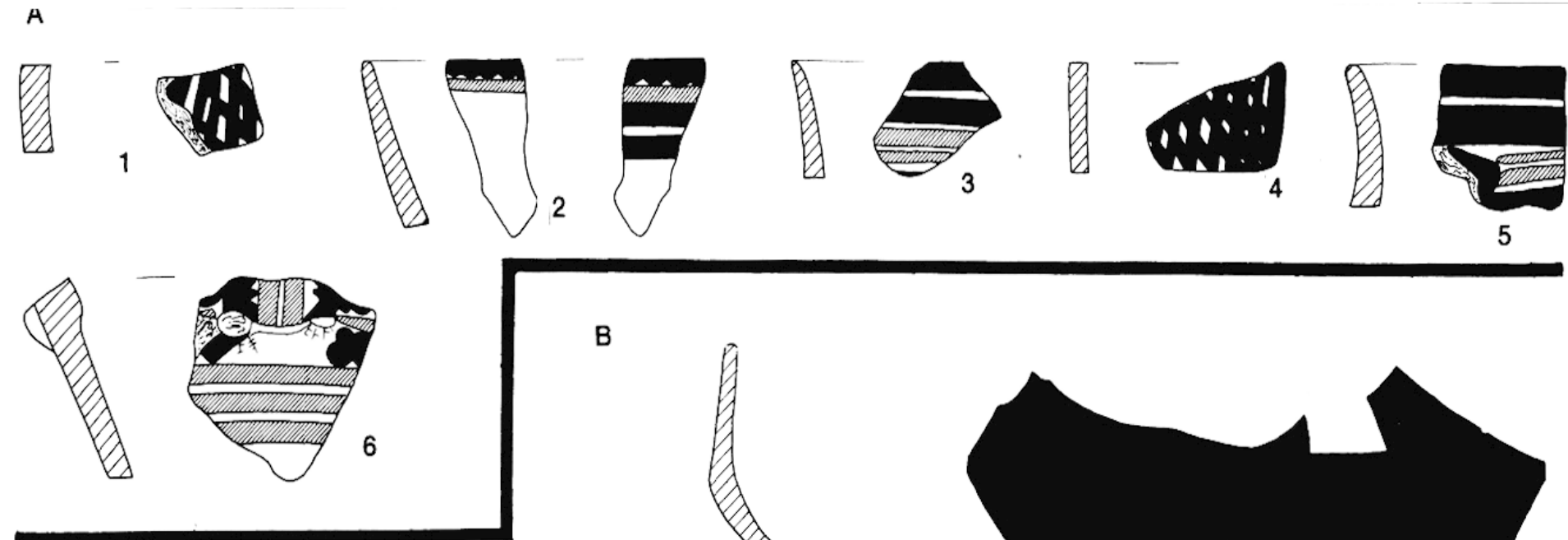

B
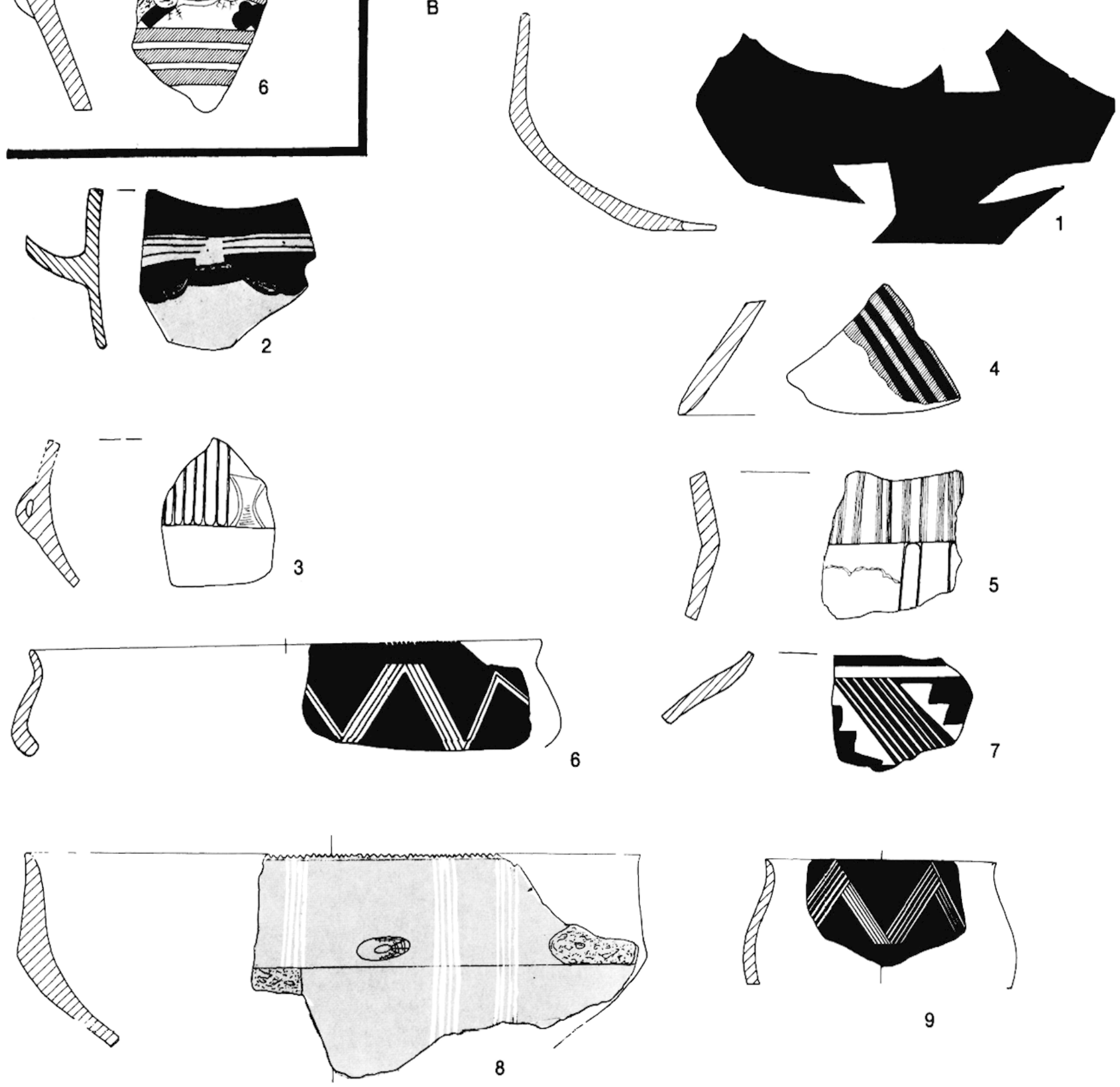

9
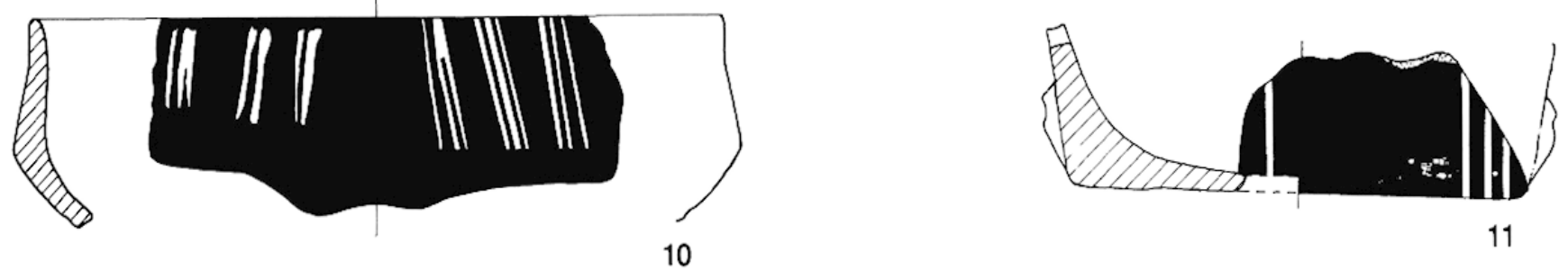

Fig. 12. - A) Céramique à décor polychrome de la période Arapi (Makrychori 2).

B) Céramique lissée noire ou brune «Larissa»: 1-5 Plateia Magoula Zarkou; 6, 8-11: Tsalmas; 7 : Kardara Magoula. Env. 1:3. 
$4 \mathrm{~m}$ à Plateia Magoula Zarkou des traces de peinture blanche, mais ils étaient trop rares et fragmentés pour permettre d'isoler une catégorie particulière. Ce n'est qu'avec la période Arapi qu'une peinture blanche sur fond rouge deviendra abondante et standardisée. Ici, les fines lignes blanches sont seulement destinées à renforcer les motifs peints en noirs et dessinant des chevrons en rubans d'un à deux $\mathrm{cm}$ de large, éventuellement découpés en métopes par des bandes verticales. La seule forme identifiable est un "compotier" à pied évasé, et il n'est sans doute pas indifférent que, comme pour le décor polychrome à bandes rouges cernées de noir, ces styles rares et élaborés paraissent réservés à des formes également exceptionnelles. A Plateia Magoula Zarkou, les fragments sont attestés entre 5,6 et 4,6 m. Les prospections du site de Tsalmas ont fourni des exemplaires de très belle facture (Éphorie de Larissa; fig. $11 \mathrm{D}$; 23, $\left.\mathrm{n}^{\circ} 1-4\right)$.

3.3.2.4. Décor polychrome de la période Arapi (fig. 12A). La période Arapi est caractérisée en particulier par une céramique polychrome à motifs noirs et rouges sur fond d'engobe blanc crayeux (Hauptmann et Milojčıć [1969], p. 60 sq. et pl. 5). Comme on l'a vu, l'avènement de cette technique est préparé par l'utilisation d'un engobe blanc également crayeux (supra $\S 3.3 .1 .4$ et 3.3.2.1) et par divers types de polychromie. Son apparition elle-même ne semble pas brutale. A Makrychori 2, elle est présente à partir du milieu des niveaux Tsangli. Quelques rares exemplaires sont attestés à Plateia Magoula Zarkou à partir de $-4,6 \mathrm{~m}$. C'est pourquoi l'on a tenu ici à mentionner pour mémoire, et pour le différencier, ce style qui sinon sort de notre corpus.

\subsection{La céramique fine noire ou brune dite Larissa.}

\subsubsection{LE PROBLÈME.}

On aborde ici le cas d'une céramique qui a été l'une des plus notables confusions des recherches sur le Néolithique européen. V. Milojčić avait en effet, en partie à bon droit, cherché à définir dans tout le Néolithique balkanique un horizon à céramique carénée noire lissée, qui aurait été lui-même contemporain du Bronze ancien anatolien, la noire brillance et le profil de la céramique étant interprétés comme une imitation de vases métalliques (MıLoJčıć [1949, 1959]). Le radiocarbone et les stratigraphies de Dikili Tach et Sitagroi, en intercalant deux millénaires et demi entre les deux horizons comparés, ont anéanti l'aspect chronologique général. Restait que si une partie du Néolithique moyen balkanique, notamment en Bulgarie et Yougoslavie, se caractérisait bien par l'avènement d'une céramique noire lissée et des formes carénées, Milojčić, tout en reconnaissant dans la phase contemporaine de Tsangli l'existence d'une telle catégorie, persistait à définir une "culture de Larissa", définie essentiellement par une telle céramique, mais placée chronologiquemeni après l'ensemble des périodes de la culture de Dimini (MrLojčıć [1959], Hauptmann [1981]). Il reprenait là d'ailleurs les conceptions précédentes de Tsountas (1908) et de WACE et Thompson (1912) qui plaçaient cette catégorie, dénommée " $\Gamma$ la», juste avant l'Âge du Bronze. Les difficultés d'un tel système, qui créait notamment en Yougoslavie un hiatus chronologique pendant toute la durée de la culture de Dimini, ont été soulignées à diverses reprises, comme il a été rappelé en introduction (Holmberg [1964], Treuil [1983], Lichardus et alii [1985]), et la preuve stratigraphique de l'identité entre "culture de Larissa" et céramique noire polie de la période Tsangli définitivement administrée par le sondage de Plateia Magoula Zarkou (Gallis [1982, 
1987]); K. Gallis proposa à cette occasion de dénommer "Tsangli-Larissa" cette phase chronologique. Bien que maintenant résolue, cette question a néanmoins quelque peu compliqué la terminologie. Le terme de céramique de "type Larissa" est à la fois d'un emploi courant et obligé, mais recouvre des réalités mouvantes où l'on doit distinguer :

a) La céramique "Larissa" classique, définie essentiellement par des coupes noires lissées, arrondies, à profil caréné ou à épaulement et un décor de cannelures, de pastilles, d'incisions incrustées ou de peinture blanche (fig. $12 \mathrm{~B}$; fig. 20, $\mathrm{n}^{\circ} 7,11,14$ ). Des descriptions exhaustives récentes en ont été données (Hauptmann [1981], Gallis [1987]).

b) Des formes identiques aux précédentes, mais de couleur différente, surtout beige ou cuir (fig. $20, \mathrm{n}^{0} 1$ ), résultat intentionnel, ou dû à une cuisson insuffisamment réductrice. Il existe aussi une céramique à extérieur noir et à intérieur rouge, notamment à Makrychori 2 , et qui préfigure la céramique monochrome rouge bien standardisée de la période Arapi.
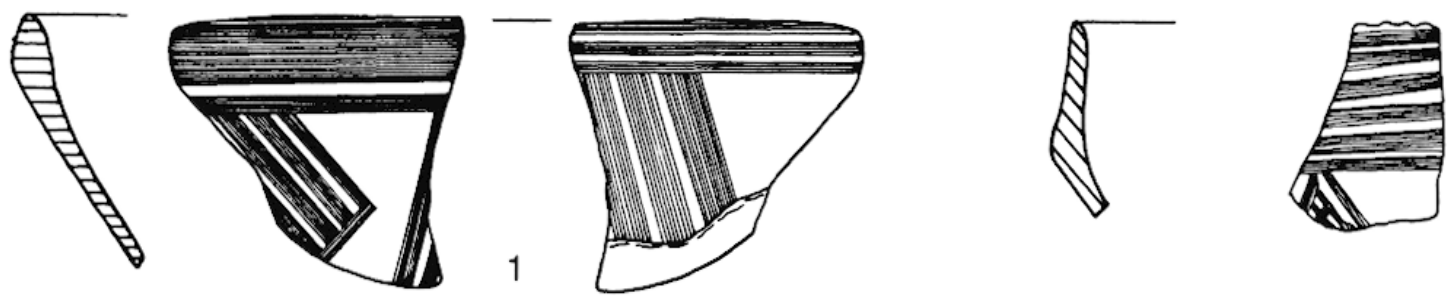

2
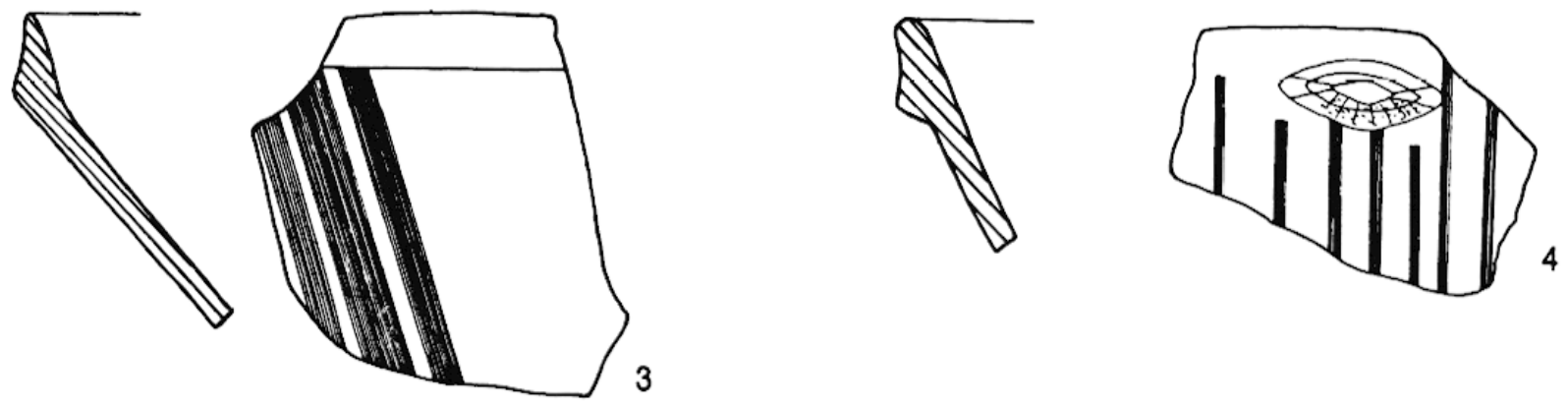

3
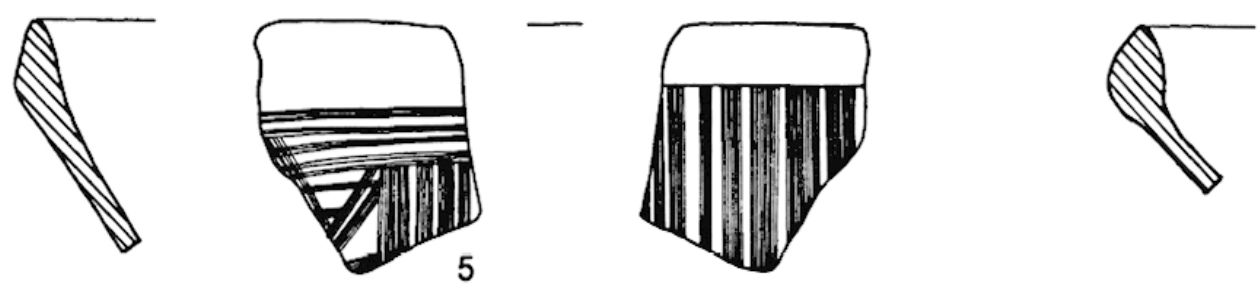

6
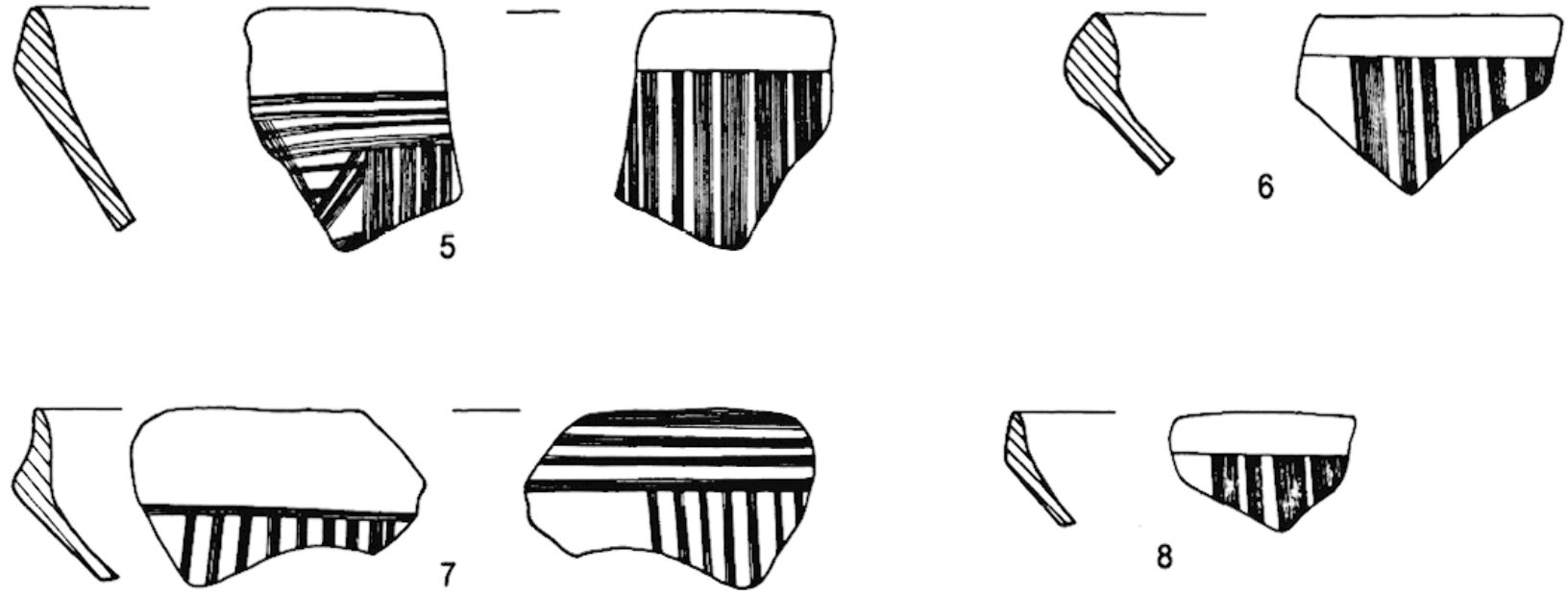

8

Fig. 13. - Céramique Tsangli à décor lissé (Makrychori 2). Env. 1:3. 
c) Une partie de la céramique noire polie et carénée de la phase Tsangli a été traditionnellement distinguée de la "culture de Larissa", notamment lorsqu'elle se trouvait dans un contexte stratigraphique indiscutable, comme à Arapi (HAUptMann et Milosč́ć [1969], p. 20-26 et pl. 1); il s'agit notamment des écuelles biconiques à carène haute (fig. 13), souvent à décor lissé, récipients qui peuvent ètre également de couleur brun-sombre.

d) La volonté de définir une "culture de Larissa" avec toutes ses composantes a parfois fait classer dans cette "culture" de la céramique peinte, voire polychrome, classique de la période Tsangli (Hauptmann [1981], pl. 9).

e) Enfin, rappelons que ces céramiques noires polies existent à différents moments du Néolithique thessalien, notamment dans le Proto-Sesklo et dans la période Arapi - cette dernière apparaissant comme un développement ultérieur de la céramique "Larissa". Dans les régions limitrophes, elles ne se limitent pas non plus à l'horizon du "Néolithique moyen" balkanique, mais perdurent, notamment en Yougoslavie et en Macédoine centrale grecque, pendant toute la durée du Néolithique récent et jusqu'au Chalcolithique.

A cela s'ajoute l'inégalité de répartition entre Thessalie orientale, où la céramique de Larissa est abondante - d'où son nom, qui ne correspond pas à un site particulier - et Thessalie occidentale, où elle est peu répandue; une répartition symétrique de celle de la céramique gris-sur-gris, et qui n'affecte pas seulement la quantité, mais aussi la typologie : les formes bitronconiques à carène haute et décor lissé sont peu fréquentes à l'Ouest.

Quant à la technique, elle semble procéder d'un "enfumage» à la cuisson (LETSCH et Noll [1983], p. 138), qui n'exclut pas l'ajout de matières grasses.

\subsubsection{FORMES ET DÉCOR.}

On traitera donc comme un tout, dénommé facultativement "Larissa ", l'ensemble de la céramique fine et mi-fine noire lissée; on considérera comme une variété proche, mais traitées à part (infra $\$ 3.6$ ), la céramique monochrome brune. On ne procèdera ici qu'à un bref rappel, formes et décors ayant fait l'objet de descriptions récentes (HauptmanN et Milojčić [1969], Hauptmann [1981], Gallis [1987]).

Les formes comprennent d'abord les écuelles. On peut distinguer assez nettement les écuelles à profil curviligne et épaulement plus ou moins marqué, et les écuelles bitronconiques à carène haute. Les premières sont le "Larissa" classique, et comportent une grande variété de décors (rangées de pastilles sous le bord ou près de l'épaulement, incisions incrustées et incisions mousses, cannelures, peinture blanche épaisse, bords encochés), quoique toujours discrets (fig. 12 B, 20). Les secondes sont le "Tsangli" classique, et se caractérisent par des décors lissés en bảndes verticales, obtenus par un lissage vigoureux au moyen d'un instrument rigide et étroit (petit galet?) dont les traces sont presque toujours visibles sur les plages brillantes lissées; on trouve également des bandes horizontales, notamment dans la partie supérieure des récipients, et plus rarement des bandes obliques (fig. 13). La technique "black topped», où la partie supérieure externe et l'intérieur du vase sont noirs, la partie inférieure externe restant claire, une technique attestée en Yougoslavie ou en Macédoine orientale à la même époque, est également employée sur ce type de récipient - et complique l'attribution typologique des fragments de petite taille. Les techniques décoratives de la céramique Larissa, comme la peinture blanche épaisse en motifs élémentaires ou les incisions, peuvent également se rencontrer dans de rares cas, qui prouvent les apparentements entre ces deux catégories. 


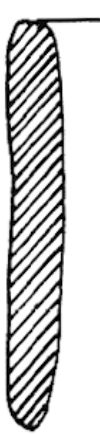

1

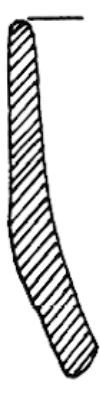

2

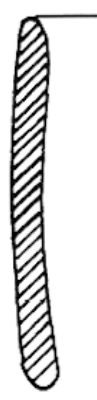

3
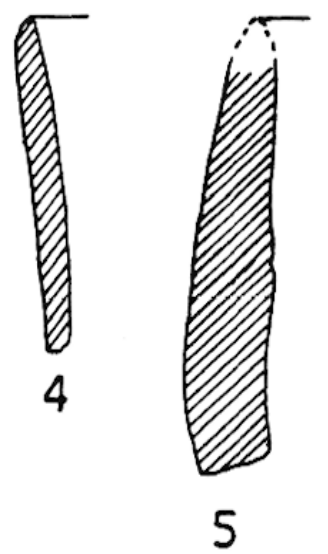

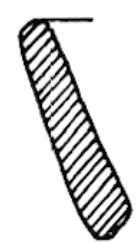

6

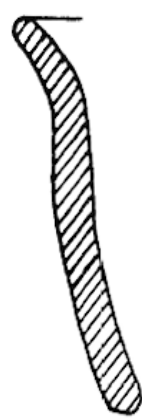

11
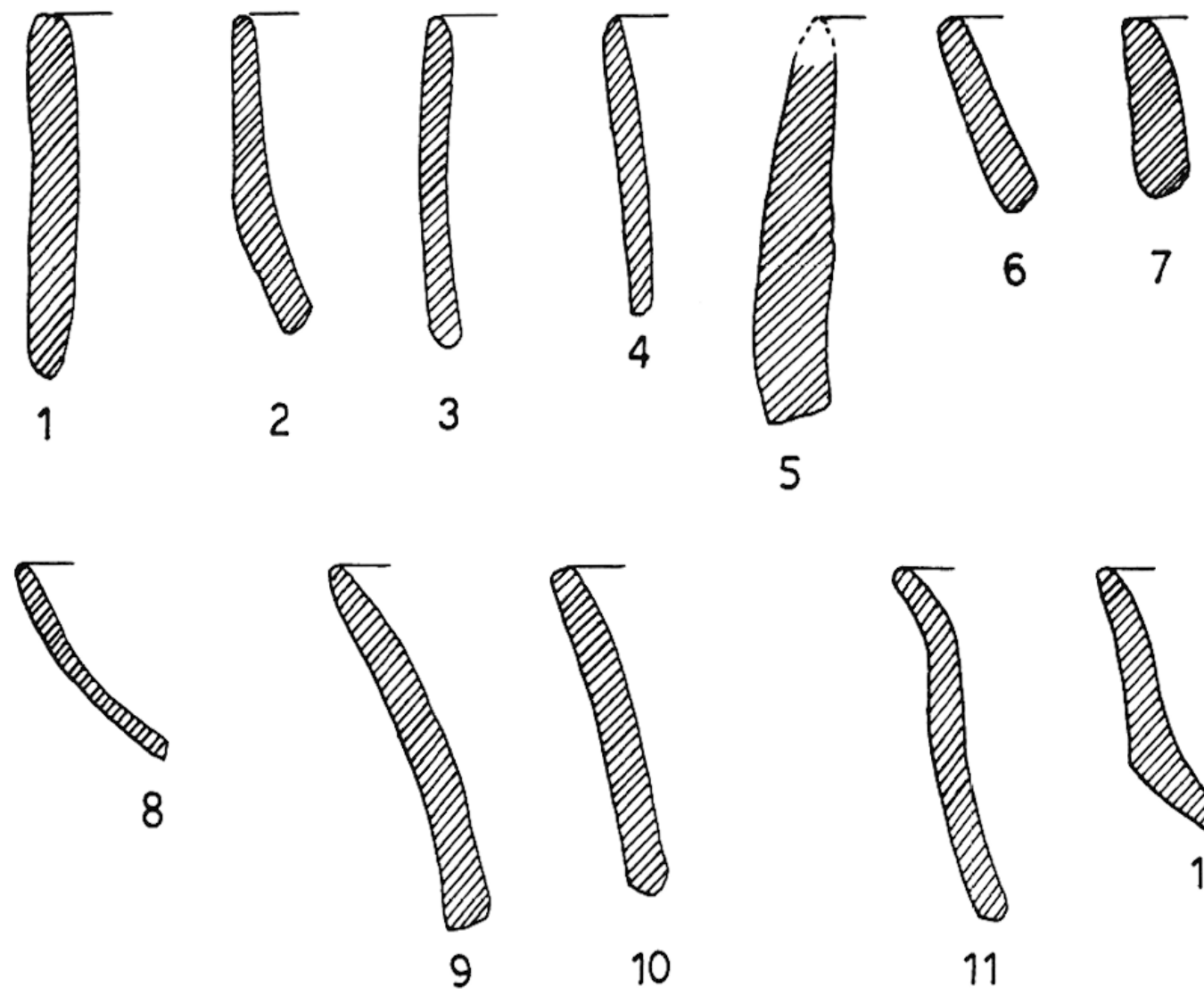

10

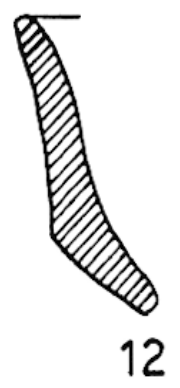

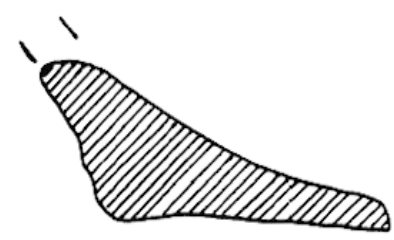

13

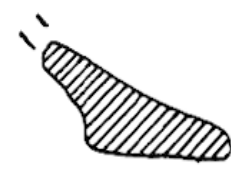

14

Fig. 14. - Échantillon de profils de la céramique grossière (Plateia Magoula Zarkou). Env. 1:3. 

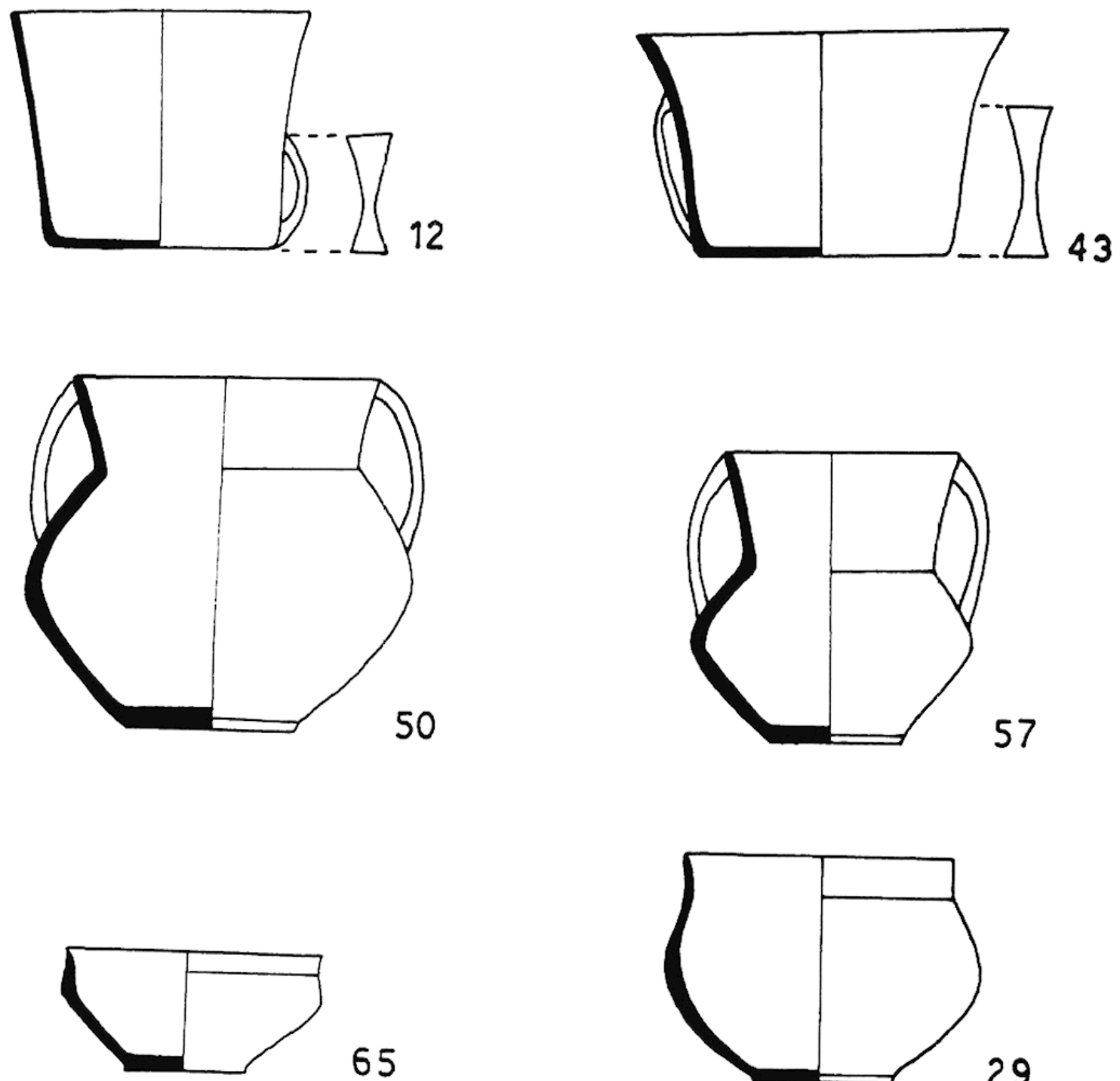

65
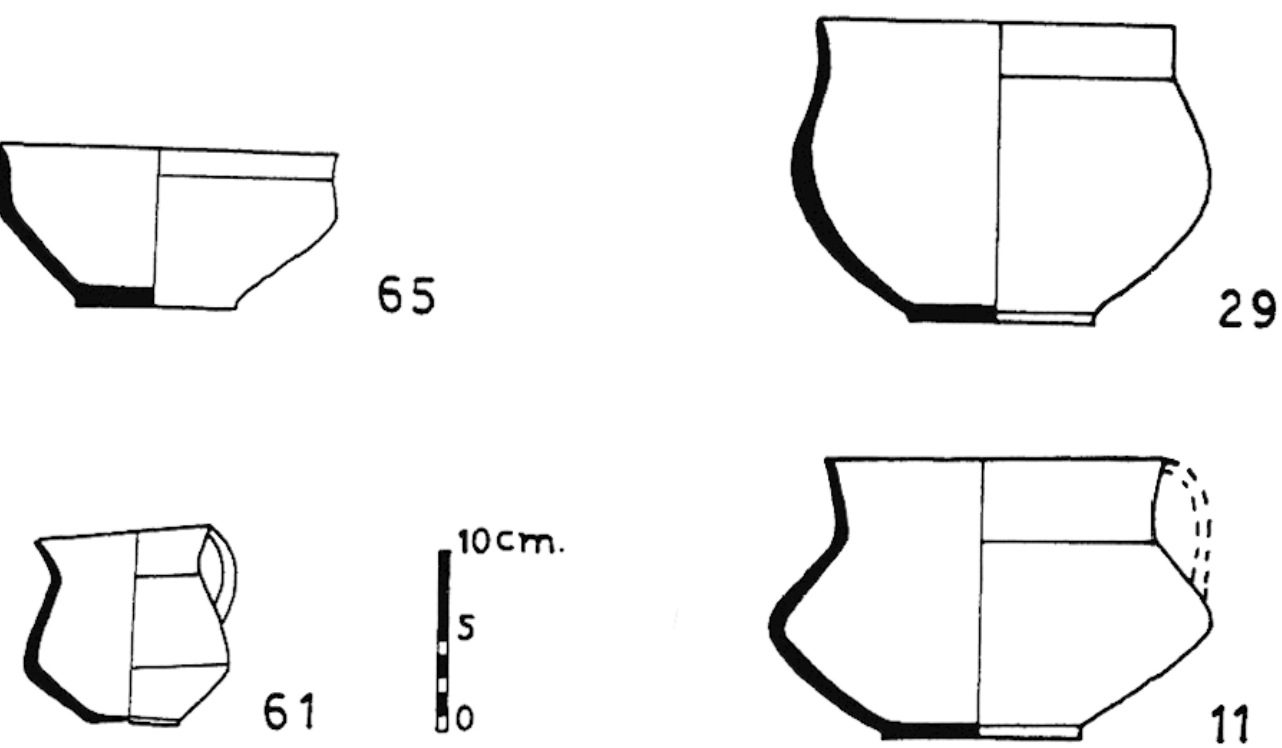

Fig. 15. - Formes de la céramique gris-sur-gris (nécropole de Plateia Magoula Zarkou). Les chiffres sont ceux du catalogue de Gall.is (1982), p. 119 sqq. Env. 1:3. 

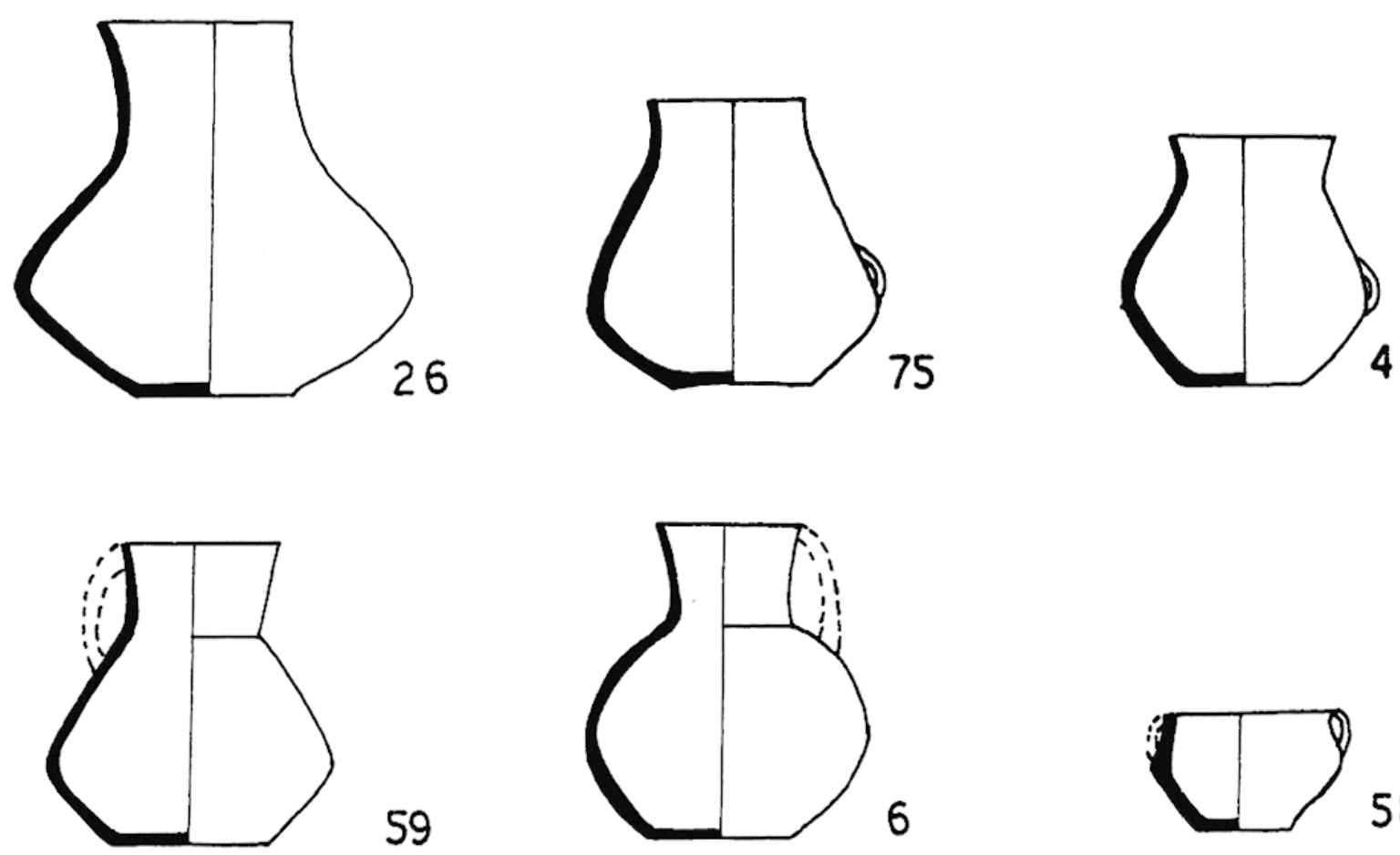

\section{9}
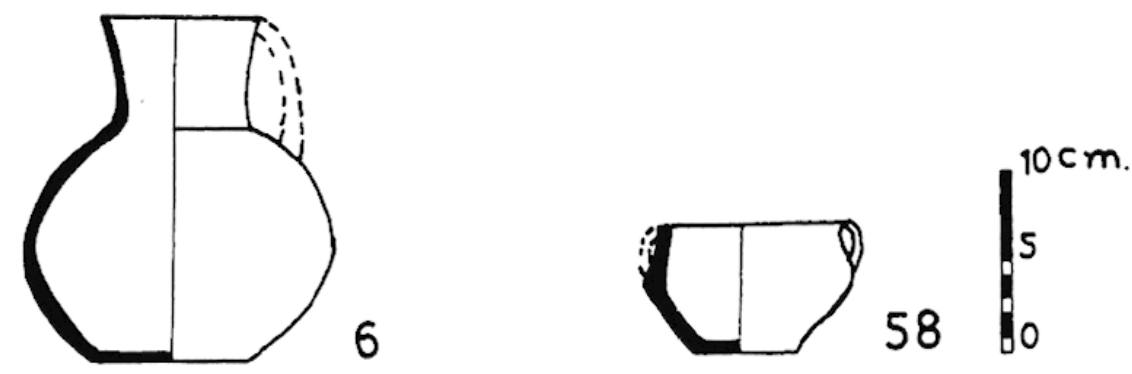

Fig. 16. - Formes de la céramique "Larissa " noire lissée (nécropole de Plateia Magoula Zarkou). Les chiffres sont ceux du catalogue de Gal.r.ss (1982), p. 119 sqq. Env. $1: 3$.

Néanmoins, le fait que ces deux catégories tendent partiellement à s'exclure sur les quelques sites fouillés est sans doute l'indice de différences chronologiques qui ont été l'une des sources des confusions susmentionnées. Dans la période Arapi, les écuelles bitronconiques tendront vers des carènes médianes.

Outre les écuelles, les bouteilles sont la seconde forme principale. Leur col est plus étroit que sur celles de la céramique gris-sur-gris, les anses verticales sont également moins fréquentes, et le point d'inflexion est plutôt situé dans la partie inférieure de la panse, lui conférant ainsi un profil piriforme (fig. 16). La panse peut être ornée de cannelures verticales. Il existe sinon quelques cas de vases à piédestal.

La valeur intrinsèque de la céramique noire polie Larissa est démontrée par sa fréquence dans les nécropoles, notamment à Soufli (BıEsantz [1959]) et à Plateia Magoula Zarkou (Gallis [1982] et infra $\S 4.4$ ), où elle est en outre associée à la céramique gris-surgris et à la céramique monochrome rouge-orange.

\subsubsection{Position stratigraphique.}

La céramique Larissa noire lissée est l'innovation techno-stylistique la plus visible qu'introduit l'émergence de Dimini ancien, et du "Néolithique moven" balkanique dans son ensemble. Elle est donc citée souvent, comme toutes les autres céramiques noires lissées contemporaines, à l'appui d'une hypothèse historique en termes de rupture, voire de migration. Cette céramique tranche à la fois avec les catégories peintes de Sesklo, et permet parfois des rapprochements avec certains sites anatoliens, comme Can Hasan (couche 2b : Titov [1969], Lichardus et alii [1985], p. 339). Dans l'état actuel de la documentation thessalienne, ces hypothèses ne peuvent cependant être ni confirmées ni infirmées. En effet, la seule stratigraphie précise de transition entre Sesklo et Dimini- 
Tsangli, celle de Plateia Magoula Zarkou, provient d'une région où cette céramique noire lissée est, on l'a vu, peu abondante, son centre de gravité se trouvant en Thessalie orientale. En revanche, les sites de Makrychori ou d'Arapi ne commencent que durant la période Tsangli-Larissa, qui connaît de toute façon, comme le "Néolithique moyen" balkanique dans son ensemble, de nombreuses implantations nouvelles à cette époque. Les arguments en faveur d'une continuité typo-technologique peuvent se fonder sur l'existence déjà mentionnée, dès les phases Proto-sesklo, et sporadiquement dans les phases Sesklo (la catégorie A5 de Wace et Thompson), d'une céramique monochrome noire polie, toutefois moins bien cuite que la céramique Larissa. Ils ne rendent compte, ni du renouvellement des formes, ni des comparaisons avec l'Anatolie. Cette discussion sera reprise en conclusion.

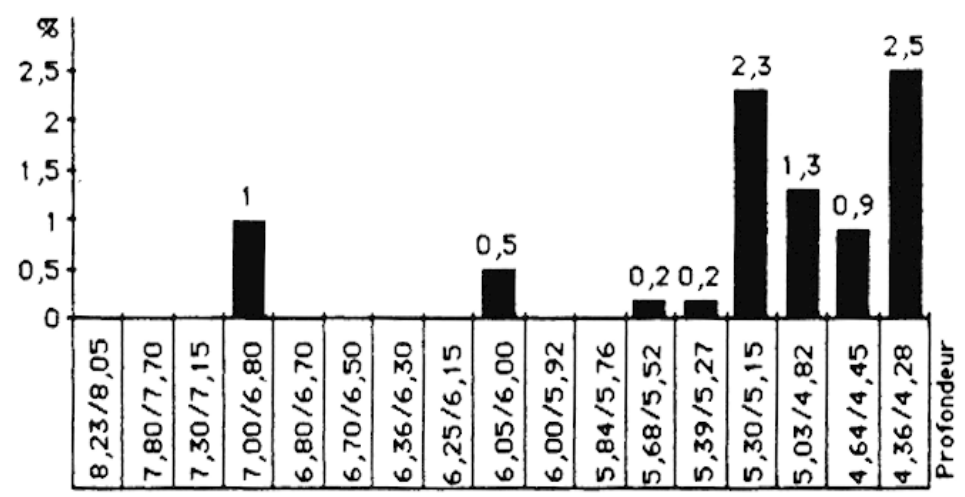

Tableau 17. - Évolution stratigraphique (en poids) à Plateia Magoula Zarkou de la céramique lissée noire ou brune de type "Larissa".

A Plateia Magoula, une céramique noire lissée, bien qu'en très faible quantité, apparaît sporadiquement dès $-7 \mathrm{~m}$. Les traits caractéristiques des types Larissa n'apparaissent cependant qu'à partir de $-6 \mathrm{~m}$, sans jamais dépasser $2,5 \%$ du total de la céramique (tabl. 17), A Makrychori, la céramique Larissa, prépondérante parmi la céramique fine (tabl. 9), est attestée dès le début de l'occupation Tsangli-Larissa (c'est-àdire dès le début de l'occupation du site). Elle reste encore présente, bien que plus faiblement, dans les niveaux Arapi, dans lesquels elle finit par disparaître, entre $-1,75$ et $1,55 \mathrm{~m}$. A Aghia Sofia également, où le sondage le plus profond s'est interrompu à l'intérieur d'une couche Arapi (MıLoJčıć et alii [1976]), une céramique noire polie sur des formes carénées est présente dans tous les niveaux, même si l'on doit faire la part d'éventuelles intrusions plus anciennes. Sur le site éponyme d'Arapi (Hauptmann et Muıo.Jč́ćc [1969]), la céramique noire polie est également prépondérante parmi la céramique fine (tabl. 18); la technique "black topped», présente à Makrychori 2 dès le début, n'est pas attestée avant les niveaux Arapi. Par ailleurs l'opposition entre Plateia Magoula Zarkou, qui connaît essentiellement des écuelles curvilignes "Larissa", de Makrychori 2 et Arapi, où les écuelles carénées bitronconiques à décor lissé sont majoritaires, traduit des différences en partie régionales, mais certainement aussi chronologiques, puisque le premier type est bien connu, par définition, dans la région de Larissa, à Otzaki notamment (Hauptmans [1981]). Dans ce cas, on peut postuler une antériorité du premier type sur le second. La séquence de Plateia Magoula Zarkou paraît en effet sans rupture, tandis que les formes carénées bitronconiques sont beaucoup plus prochẹ des formes Arapi que les écuelles curvilignes et que les stratigraphies de Makrychori 2 comme d'Arapi paraissent évoluer sans rupture vers la période Arapi proprement dite. 


\begin{tabular}{|c|c|c|c|c|c|c|c|c|c|c|}
\hline & \multicolumn{10}{|c|}{ Planum } \\
\hline & 16 & 15 & 14 & 13 & 12 & 11 & 10 & 9 & 8 & 7 \\
\hline Cèr. noire lissée int. noir... & 34,5 & 0 & 74,2 & 30,5 & 16,7 & 31 & 15,1 & 22,3 & 14,2 & 4,2 \\
\hline Cér. noire lissée int. rouge $\ldots \ldots \ldots$ & 0 & 0 & 0 & 0 & 0 & 5,1 & 5 & 19,2 & 8,3 & 10,1 \\
\hline Cér. noire lissée int. cuir.......... & 0 & 0 & 1,6 & 0 & 0 & 0 & 0 & 0 & 0 & 3,3 \\
\hline Cér. cuir lissée int. cuir........... & 14,5 & 0 & 0 & 10,2 & 7,2 & 4,9 & 1 & 0 & 0 & 0 \\
\hline Cér. cuir lissée int. rouge $\ldots \ldots \ldots$ & 0 & 0 & 0 & 0 & 1,7 & 3,3 & 13,6 & 2,5 & 6,1 & 33,1 \\
\hline Cér. cuir lissée int. noir............ & 0 & 0 & 0 & 0 & 5 & 4,5 & 4,5 & 10 & 5,5 & 0 \\
\hline Total céramique "Larissa & 49,1 & 0 & 75,8 & 40,7 & 30,6 & 48,8 & 39,3 & 54 & 34 & 50,1 \\
\hline Cér. peinte mate brun sur brun ..... & 0 & 9,5 & 7,8 & 11,9 & 10,6 & 15,9 & 17,6 & 2,8 & 1,4 & 0,7 \\
\hline Cér. peinte noire sur rouge $\ldots \ldots \ldots$ & 9,1 & 0 & 0 & 1,8 & 0 & 0 & 6 & 0,6 & 0,4 & 0 \\
\hline Cér. polychrome $\ldots \ldots \ldots \ldots \ldots$ & 0 & 0 & 0 & 0 & 0 & 0 & 0 & 1,7 & 0,8 & 1,9 \\
\hline Cér. peinte en brun sur engobe blanc. & 0 & 0 & 0 & 0 & 3,9 & 0,8 & 1 & 1,4 & 0 & 6 \\
\hline Cér. incisée.......... & 0 & 0 & 0 & 7,1 & 7,2 & 3,5 & 1,3 & 2,2 & 0 & 1,1 \\
\hline Cér. gris-sur-gris $\ldots \ldots \ldots \ldots$ & 0 & 0 & 0 & 1,8 & 0 & 0,4 & 0 & 0 & 0 & 0 \\
\hline Cér. gris-noirâtre $\ldots \ldots \ldots \ldots \ldots \ldots$ & 0 & 0 & 0 & 0 & 3,3 & 0 & 9,1 & 1,4 & 3,2 & 0 \\
\hline Cér. monochrome $\ldots \ldots \ldots \ldots \ldots$ & 0 & 0 & 5,5 & 0,4 & 4,4 & 0,4 & 6 & 8,1 & 10,3 & 5,8 \\
\hline Cér. grossière. & 41,8 & 90,5 & 10,9 & 36,3 & 40 & 30,2 & 19,6 & 27,9 & 49,8 & 35,8 \\
\hline
\end{tabular}

Tableau $18 \mathrm{a}$. - Pourcentages (en poids) des différentes catégories céramiques dans les niveaux "TsangliLarissa " du site d'Arapi (d'après le matériel conservé à l'éphorie de Larissa).

\begin{tabular}{|c|c|c|c|c|c|}
\hline \multicolumn{2}{|c|}{ SONDAGES } & $\begin{array}{l}\text { CÉRAMIQUE } \\
\text { GROSSIÈRE }\end{array}$ & $\begin{array}{l}\text { Céramique } \\
\text { Peinte }\end{array}$ & $\begin{array}{l}\text { CÉRAMIQUE } \\
\text { MONOCHROME } \\
\text { SOMBRE }\end{array}$ & TOTAL \\
\hline \multirow{2}{*}{ 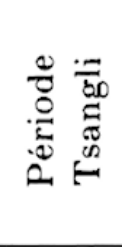 } & II & $\begin{array}{l}500 \\
\quad 70 \% \\
\end{array}$ & 42 & $\begin{array}{ll}175 & \\
& 24 \% \\
\end{array}$ & 720 \\
\hline & I & $\begin{array}{l}>500 \\
>57 \% \\
\end{array}$ & $\begin{array}{r}<64 \\
<7 \% \\
\end{array}$ & $\begin{array}{l}<300 \\
\quad<34 \% \\
\end{array}$ & $>864$ \\
\hline \multirow{2}{*}{ 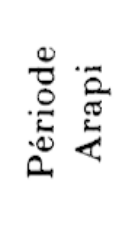 } & I & $\begin{array}{r}270 \quad \\
\quad 45 \% \\
\end{array}$ & $5 \%$ & $\begin{array}{r}292 \\
\quad 49 \% \\
\end{array}$ & 593 \\
\hline & II & $\begin{array}{l}>750 \\
>45 \%\end{array}$ & $\begin{array}{r}<96 \\
<6 \%\end{array}$ & $\begin{array}{l}<816 \\
\quad<49 \%\end{array}$ & $>1662$ \\
\hline
\end{tabular}

TABLEAU $18 \mathrm{~b}$. - Pourcentages des différentes catégories céramiques pour les niveaux "Tsangli-Larissa" et "Arapi des deux sondages (I et II) du site d'Arapi (d'après Hauptmann et Mılojčıć [1969]). 


\subsection{LES DÉCORS INCISÉS, IMPRIMÉS OU PLASTIQUES.}

On sait que la culture de Sesklo utilise, surtout dans ses phases anciennes, des décors imprimés à l'aide d'un poinçon ou d'un peigne à pointes mousses, décors issus de la tradition antérieure Proto-Sesklo et Pré-Sesklo-Magoulitsa (Hourmouziadis [1971]), ainsi que des décors incisés, le plus souvent combinés avec de la peinture et de l'impression, et souvent sur des formes particulières (par exemple Motrier [1981], pl. D). Plus tard, dans Dimini récent, il existe une céramique incisée très caractéristique. En revanche les périodes Tsangli et Arapi semblent ignorer presque totalement ces techniques, qui sont bien attestées à la même époque, non seulement en Crète, mais dans le reste des Balkans, notamment dans la culture de Vinča et dans les groupes du Bas-Danube. C'est pourquoi la présence très sporadique de ces techniques peut tenir à des intrusions. Ainsi à Plateia Magoula Zarkou, où la culture de Sesklo est présente pour une grande partie de son développement, les rares fragments présents dans les phases terminales de cette culture (tabl. 19) peuvent être issus de couches plus anciennes.

On mentionnera néanmoins, pour les décors incisés, quelques rares fragments à motifs incisés en chevrons, avec une technique rudimentaire sur une pâte claire mi-fine, et sinon le décor incisé, cannelé ou à rebord encoché de la céramique du type Larissa. Quant aux décors plastiques, ils sont présents, outre de très fins cordons lisses associès à la céramique à décor frotté (supra $\$ 3.1 .2 .1$ d), sur de la céramique grossière à paroi épaisse, surtout, à Plateia Magoula Zarkou, à partir de $-6 \mathrm{~m}$. De tels cordons, parfois à impressions digitales, se retrouvent à Arapi, dans les niveaux Tsangli puis Arapi (Hauptmann et Mılojč̀ć [1969], p. 76 et pl. XI).

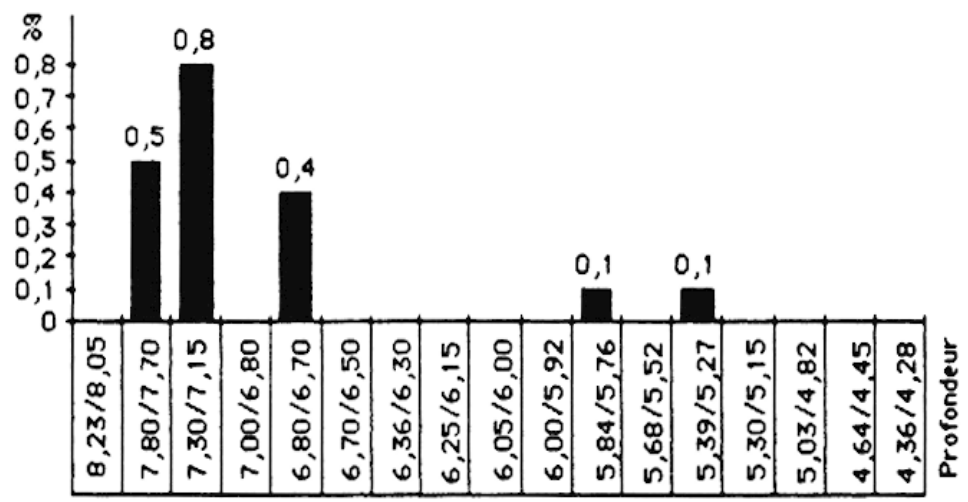

Tableau 19. - Évolution stratigraphique (en poids) de la céramique à décor incisé, imprimé ou plastique sur le site de Plateia Magoula Zarkou.

\subsection{La CÉramique MONOCHROME FINE ET MI-FINE.}

Entre la céramique décorée et la céramique grossière, il existe une catégorie non décorée intermédiaire importante, dont les différentes variétés subissent des évolutions chronologiques visibles (tabl. 20). Par la finesse de la pâte et le traitement de surface, ces céramiques sont parfois proches des catégories décorées, et peuvent d'ailleurs être les mêmes : fragment non décoré d'un vase décoré, ou récipient non décoré mais de technique identique. Ce fait est confirmé par leur usage social, puisqu'un tiers des 
récipients de la nécropole de Plateia Magoula Zarkou en sont issus. La céramique monochrome varie stratigraphiquement de manière "gaussienne» inversée : elle représente en effet près de $30 \%$ de la céramique dans les niveaux les plus anciens, baisse rapidement jusqu'à $4 \%$ dans les niveaux moyens, et remonte à $15-20 \%$ dans les niveaux supérieurs; cette évolution est parallèle à celle de la céramique décorée dans son ensemble, et évidemment inverse de celle de la céramique grossière (infra $\$ 3.7$.). On peut distinguer :

a) pour mémoire, la céramique noire lissée "Larissa", dont une partie est non décorée. Il s'agit de la seule qui soit nettement à cuisson réductrice, mais il laut aussi mentionner la variante brun cuir, qui peut parfois appartenir à des vases "black topped", et la variante à intérieur rouge, catégories surtout présentes à Makrychori 2 et Arapi. La céramique Larissa est normalement d'une facture plus fine, par sa pâte et son traitement de surface, que les autres catégories décrites cidessous.

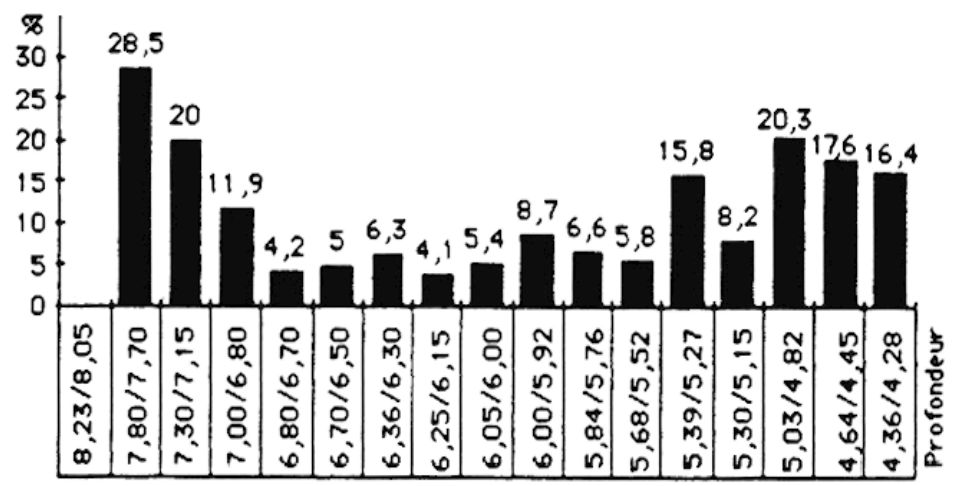

Tableau 20. - Evolution stratigraphique (en poids) de la céramique monochrome fine et mi-fine sur le site de Plateia Magoula Zarkou.

b) une céramique orange, ou orange rosâtre (Munsell 5 YR 7/2). Cette catégorie représente à Plateia Magoula Zarkou 2 à $7 \%$ du total de la céramique et la majorité de la céramique monochrome dans les niveaux où elle est bien attestée, c'est-à-dire jusque vers $5,8 \mathrm{~m}$; dans les niveaux moyens, entre 7 et $6 \mathrm{~m}$ environ, là où la céramique monochrome connaît un fort recul, elle en représente même la quasi totalité (tabl. 21). Sa facture est comparable à celle de la céramique à décor frotté, tout comme les formes reconstituables : gobelets hauts à parois fines et légèrement sinueuses, et bouteilles à col court et parois plus épaisses. De fait, dans le niveau le plus ancien

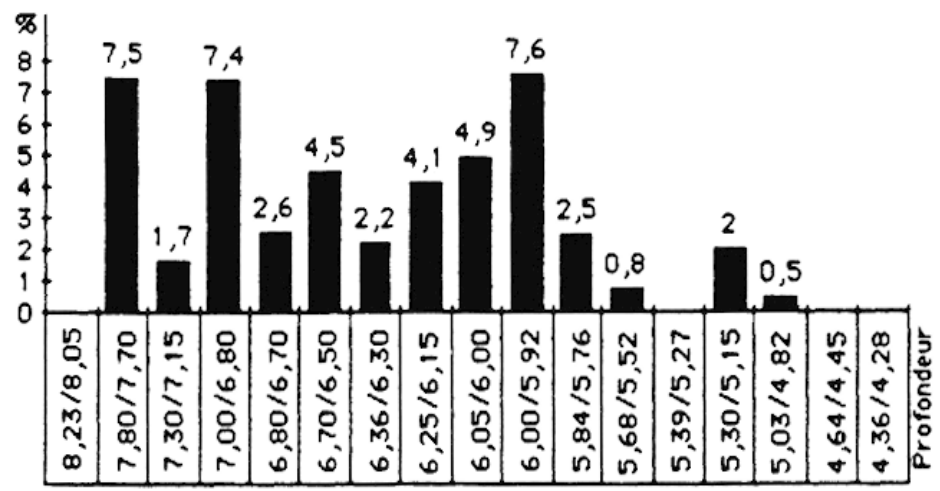

TABLEAU 21. - Évolution stratigraphique (en poids) de la céramique monochrome orange fine et mi-fine sur le site de Plateia Magoula Zarkou. 
Fig. 18. - Céramique peinte en brun-rouge sur clair "classique» (nos 2-3) ou "dégénérée» (nos 1, 4-8). Plateia - Magoula Zarkou : 1 : 6,5-6,2 m; 2-3: 7-6,8 m; $4: 5,3-5,15 \mathrm{~m} ; 5: 5-5,4 \mathrm{~m} ; 6$ : 5,3-5,2 m; $7: 5,6-5,5 \mathrm{~m}$; $8: 4,8-4,7 \mathrm{~m}$.

Fig. 19. - Céramique à décor frotté (Plateia Magoula Zarkou). Grattage présumé : nos 2, 4, 8-9; peinture présumée : nos 1, 3,5-7, 10. Profondeurs : $1: 6,3-6,2 \mathrm{~m} ; 2: 5-4,9 \mathrm{~m} ; 3-4: 5,7-5,5 \mathrm{~m} ; 5: 6-5,9 ; 6: 5,5-5,4 \mathrm{~m}$; $7: 5,8-5,7 ; 8: 6,7-6,5 ; 9: 6-5,9 ; 10: 6,3-6,2$.

Fig. 20. - Céramique grise ou noire de Plateia Magoula Zarkou ( $\left.n^{\circ *} 2-6,8-10,12-13\right)$, Tsalmas ( $\left.n^{\circ *} 1,11,14\right)$ et Kardara (n* 7). 1 : Céramique "Larissa" couleur cuir; 2 : Céramique "proto-gris-sur-gris" $(6,2-6,1 \mathrm{~m})$; 3 : Céramique "proto-gris-sur-gris" $(5,2-5 \mathrm{~m}) ; 4$ : Céramique gris-sur-gris $(4,6-4,4 \mathrm{~m}) ; 5$ : Céramique "proto-grissur-gris" $(6,2-6,1 \mathrm{~m}) ; 6$ : Céramique gris-sur-gris $(4,7-4,6 \mathrm{~m}) ; 7$ : Céramique "Larissa noire peinte en blanc; 8 : Céramique gris-sur-gris $(5-4,8 \mathrm{~m}) ; 9$ : Céramique gris-sur-gris $(5-4,8 \mathrm{~m}) ; 10$ : Céramique gris-sur-gris $(4,8-4,7 \mathrm{~m}) ; 11$ : Céramique "Larissa" noire peinte en blanc; 12 : Céramique gris-sur-gris $(5-4,8 \mathrm{~m})$;

13 : Céramique gris-sur-gris $(4,6-4,4 \mathrm{~m}) ; 14$ : Céramique «Larissa" noire peinte en blanc.

Fig. 21. - Céramique peinte de la phase "Tsangli-Larissa" (Plateia Magoula Zarkou, sauf no 3 de Tsalmas). Peinture noire sur rouge ( ${ }^{\text {os }} 3,5,7,8,11,12$ ); Peinture brune sur brun clair ( $\left.n^{\text {os }} 1,4,9,10\right)$; Peinture brune sur engobe blanc (nos 2,6$) .1: 4,4-4,3 \mathrm{~m} ; 2: 5-4,8 \mathrm{~m} ; 3:$ Tsalmas $; 4: 4,8-4,7 \mathrm{~m} ; 5: 5,5-5,3 \mathrm{~m} ; 6: 4,6-4,4 \mathrm{~m}$; $7: 4,6-4,4 \mathrm{~m} ; 8: 4,5-4,3 \mathrm{~m}: 9: 4,3-4,2 \mathrm{~m} ; 10: 5-4,8 \mathrm{~m} ; 11: 5,5-5,4 \mathrm{~m} ; 12: 4,6-4,4 \mathrm{~m}$.

Fig. 22. - Céramique peinte en rouge avec triangles noirs (Plateia Magoula Zarkou). Profondeurs : $1: 5,7-$ 5,5 m; $2: 5,3-5,2 \mathrm{~m} ; 3: 5,8-5,7 \mathrm{~m} ; 4: 5,4-5,3 \mathrm{~m} ; 5: 5,5-5,4 \mathrm{~m} ; 6: 5,4-5,3 \mathrm{~m} ; 7: 5,2-5 \mathrm{~m} ; 8: 5,4-5,3 \mathrm{~m}$.

Fig. 23. - Céramique polychrome. Vos 1-2, 4-5: Tsalmas; n“ 3 : Plateia Magoula Zarkou (6,05-6 m).

Fig. 24. - Figurines anthropomorphes en technique gris-sur-gris; n" 1 : Petrino; n" 2 : Vikaia.

Fig. 25. - 1 : Forme et décor de transition entre Sesklo et Tsangli-Larissa (trouvaille de surface, Magoula Aghiou Georghiou, Sofades); 2 : céramique peinte en noir sur rouge, Orphana; 3 : céramique gris-sur-gris, Platykambos. 


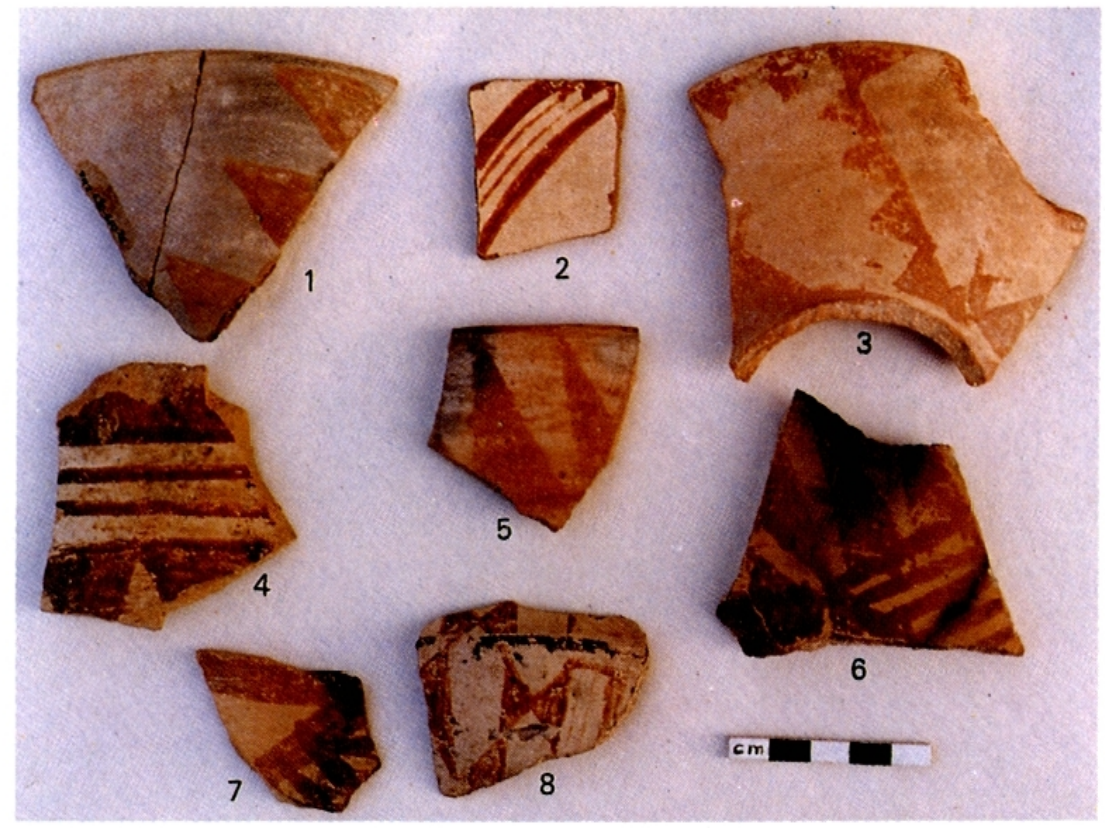

Fig. $18, n^{\circ} 3=$ fig. $4, n^{\circ} 2-n^{\circ} 6=$ fig. $4, n^{\circ} 3-n^{\circ} 8=$ fig. $4, n^{\circ} 1$.
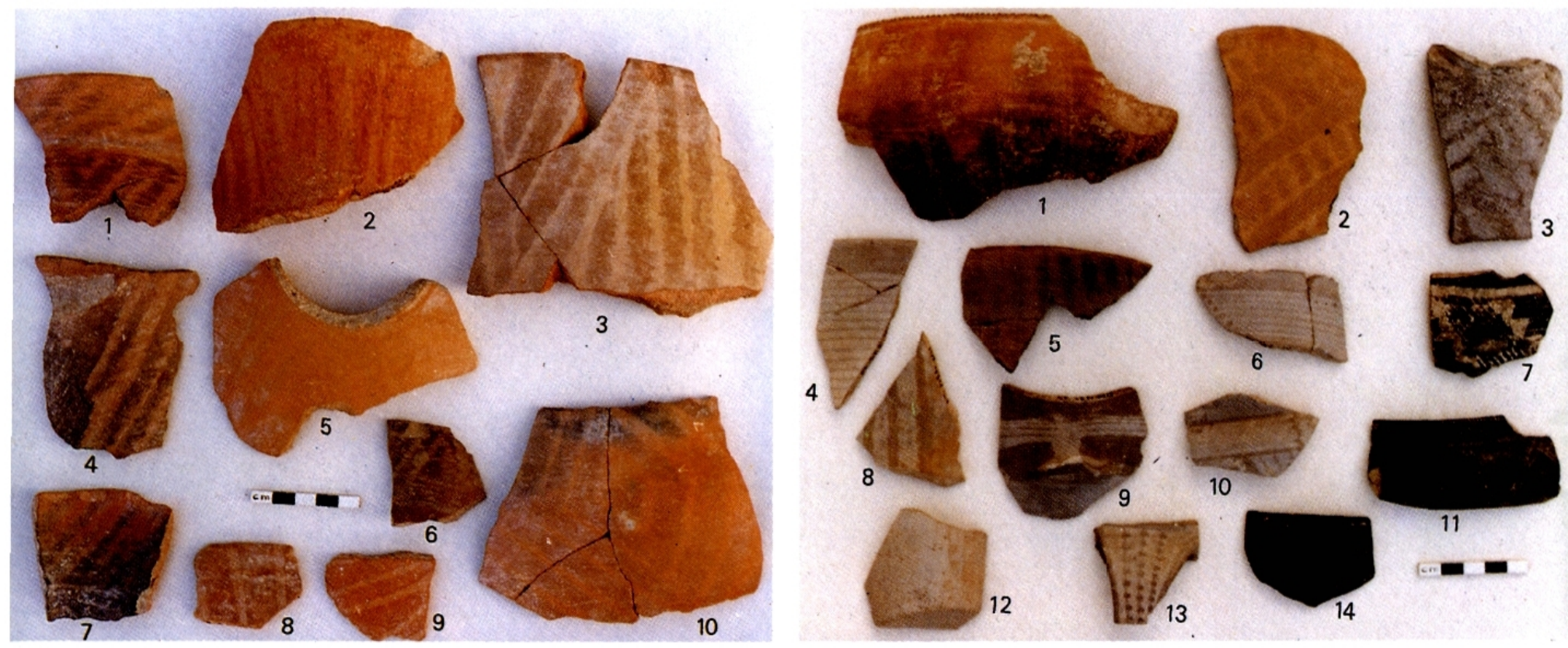

ig. $19, \mathrm{n}^{\circ} 3=$ fig. $6, \mathrm{n}^{\circ} 1-\mathrm{n}^{\circ} 4=$ fig. $5, \mathrm{n}^{\circ} 1-\mathrm{n}^{\circ} 5=$ fig. 6, Fig. $20, \mathrm{n}^{\circ} 1=$ fig. $12 \mathrm{~B}, \mathrm{n}^{\circ} 8-\mathrm{n}^{\circ} 2=$ fig. $7 \mathrm{~A}, \mathrm{n}^{\circ} 2-\mathrm{n}^{\circ} 3=$ $\mathrm{n}^{\circ} 9-\mathrm{n}^{\circ} 7=$ fig. $6, \mathrm{n}^{\circ} 2$.

fig. $7 \mathrm{~B}, \mathrm{n}^{\circ} 1-\mathrm{n}^{\circ} 4=$ fig. $7 \mathrm{~B}, \mathrm{n}^{\circ} 6-\mathrm{n}^{\circ} 5=$ fig. 8,3 $-\mathrm{n}^{\circ} 6=$ fig. $8, \mathrm{n}^{\circ} 2-\mathrm{n}^{\circ} 7=$ fig. $12 \mathrm{~B}, 7-\mathrm{n}^{\circ} 9=$ fig. $12 \mathrm{~B}, 2-\mathrm{n}^{\circ} 10=$ fig. $7 \mathrm{~B}, 4-\mathrm{n}^{\circ} 11=$ fig. $12 \mathrm{~B}, 6$ $-\mathrm{n}^{\circ} 12=$ fig. $7 \mathrm{~B}, 5-\mathrm{n}^{\circ} 13=$ fig. $8, \mathrm{n}^{\circ} 1-\mathrm{n}^{\circ} 14=$ fig. $12 \mathrm{~B}, \mathrm{n}^{\circ} 9$.
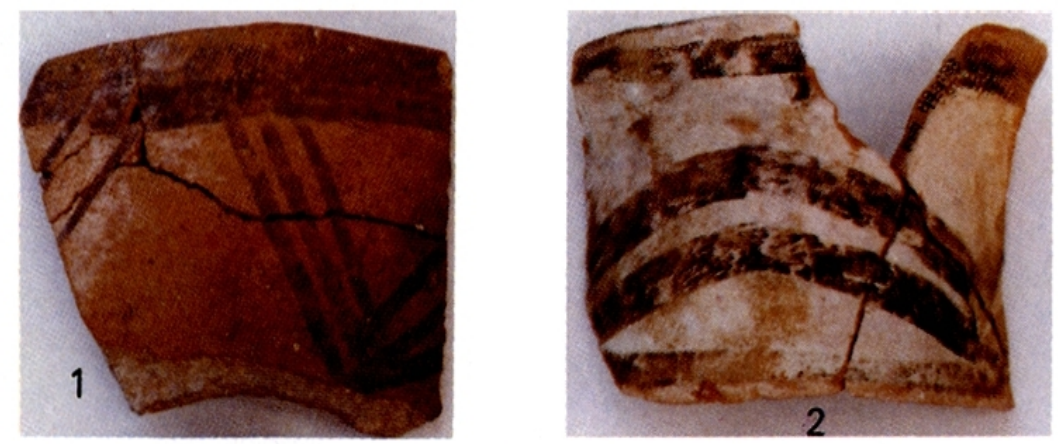

Fig. $21, \mathrm{n}^{\circ} 2=$ fig. $11 \mathrm{~A}, \mathrm{n}^{\circ} 1-\mathrm{n}^{\circ} 3=$ fig. $9 \mathrm{~A}, \mathrm{n}^{\circ} 10-\mathrm{n}^{\circ} 4=$ fig. $9 \mathrm{~B}, \mathrm{n}^{\circ} 3-\mathrm{n}^{\circ} 5=$ fig. $9 \mathrm{~A}, \mathrm{n}^{\circ} 6-\mathrm{n}^{\circ} 7=$ fig. $9 \mathrm{~A}, \mathrm{n}^{\circ} 8$ $-\mathrm{n}^{\circ} 8=$ fig. $9 \mathrm{~A}, \mathrm{n}^{\circ} 9-\mathrm{n}^{\circ} 10=$ fig. $9 \mathrm{~B}, \mathrm{n}^{\circ} 1-\mathrm{n}^{\circ} 12=$ fig. $9 \mathrm{~A}, \mathrm{n}^{\circ} 2$.

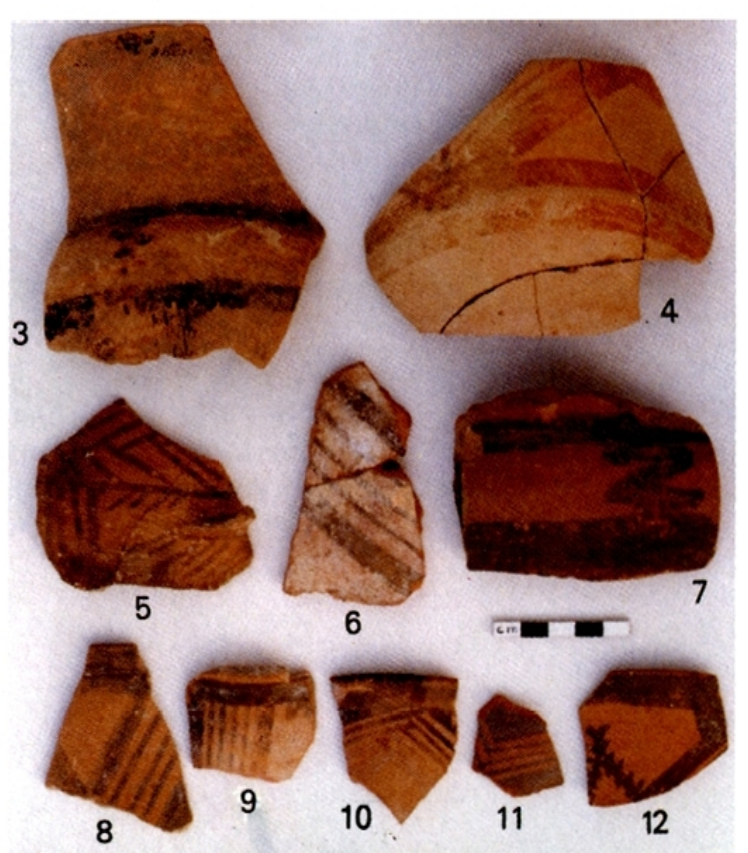




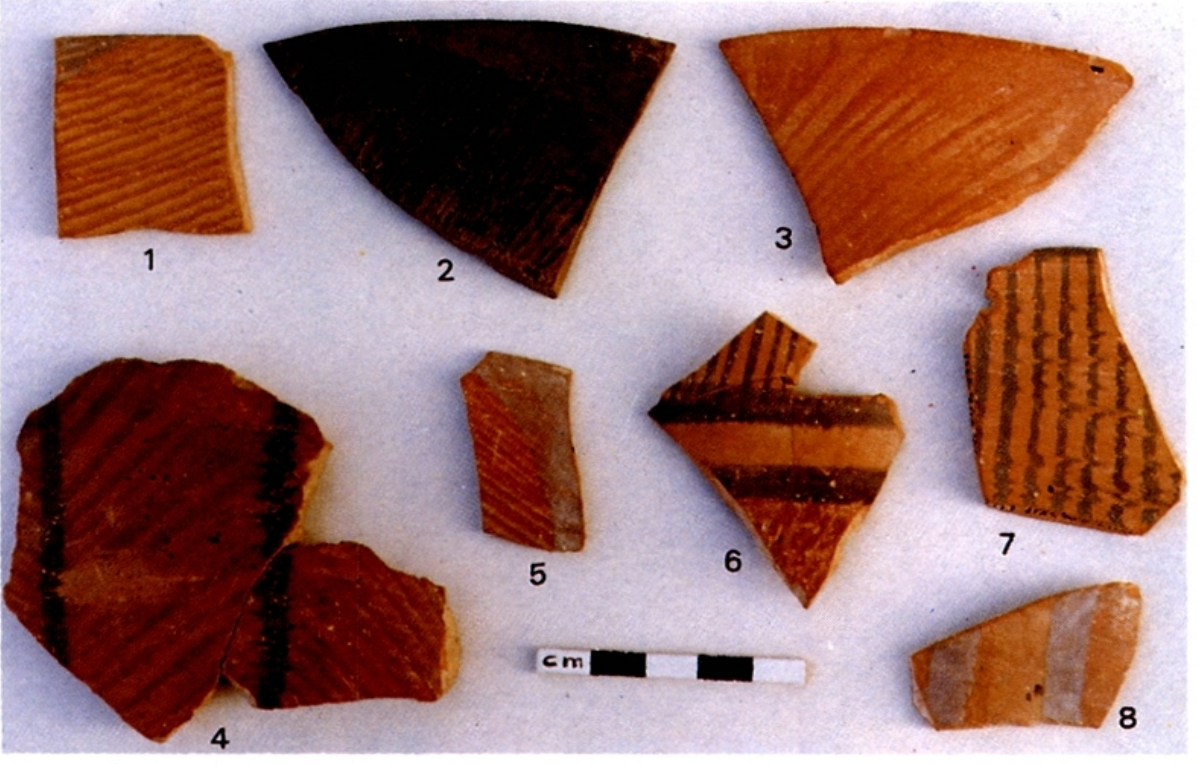

Fig. $22, n^{\circ} 1=$ fig. $11 \mathrm{C}, \mathrm{n}^{\circ} 6-\mathrm{n}^{\circ} 2=$ fig. $11 \mathrm{C}, \mathrm{n}^{\circ} 5-\mathrm{n}^{\circ} 4=$ fig. $11 \mathrm{C}$, $n^{\circ} 4-n^{\circ} 6=$ fig. $11 \mathrm{C}$, no $^{\circ} \quad-n^{\circ} 7=$ fig. $11 \mathrm{C}, n^{\circ} 1$.

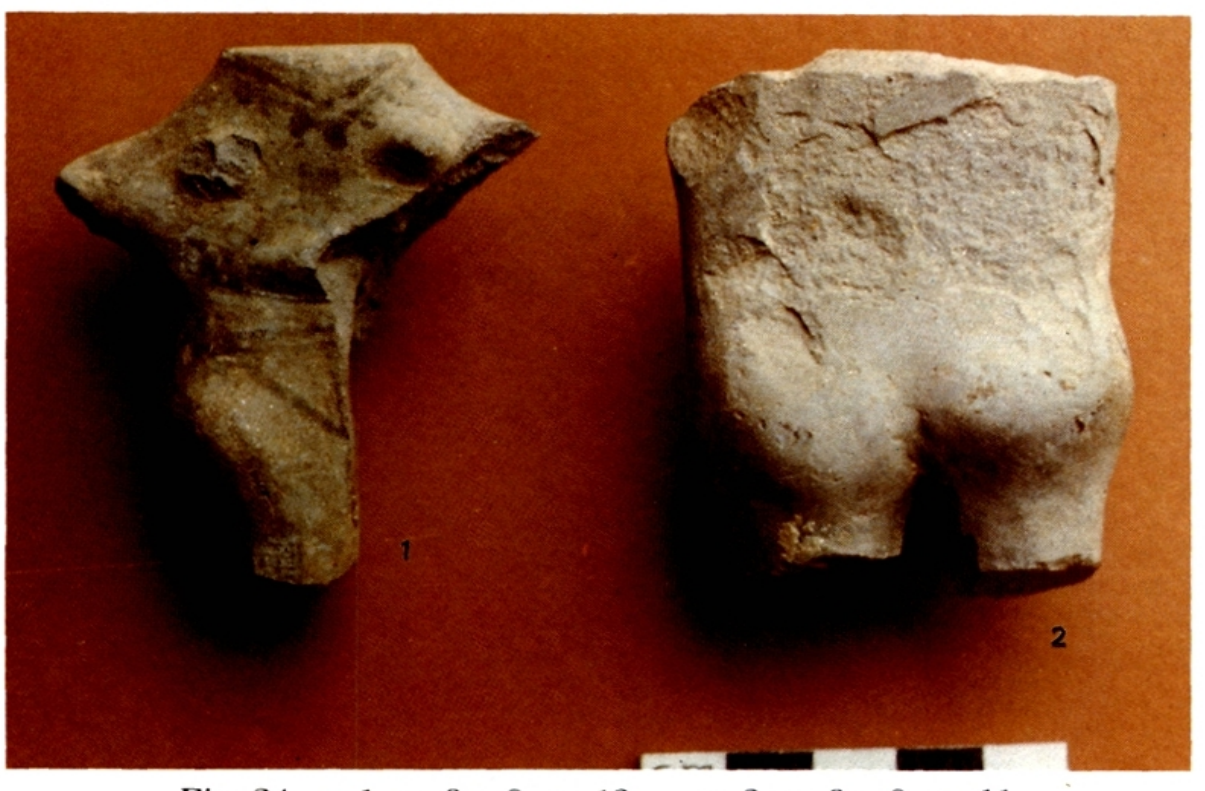

Fig. $24, \mathrm{n}^{\circ} 1=$ fig. $8, \mathrm{n}^{\circ} 12-\mathrm{n}^{\circ} 2=$ fig. $8, \mathrm{n}^{\circ} 11$.

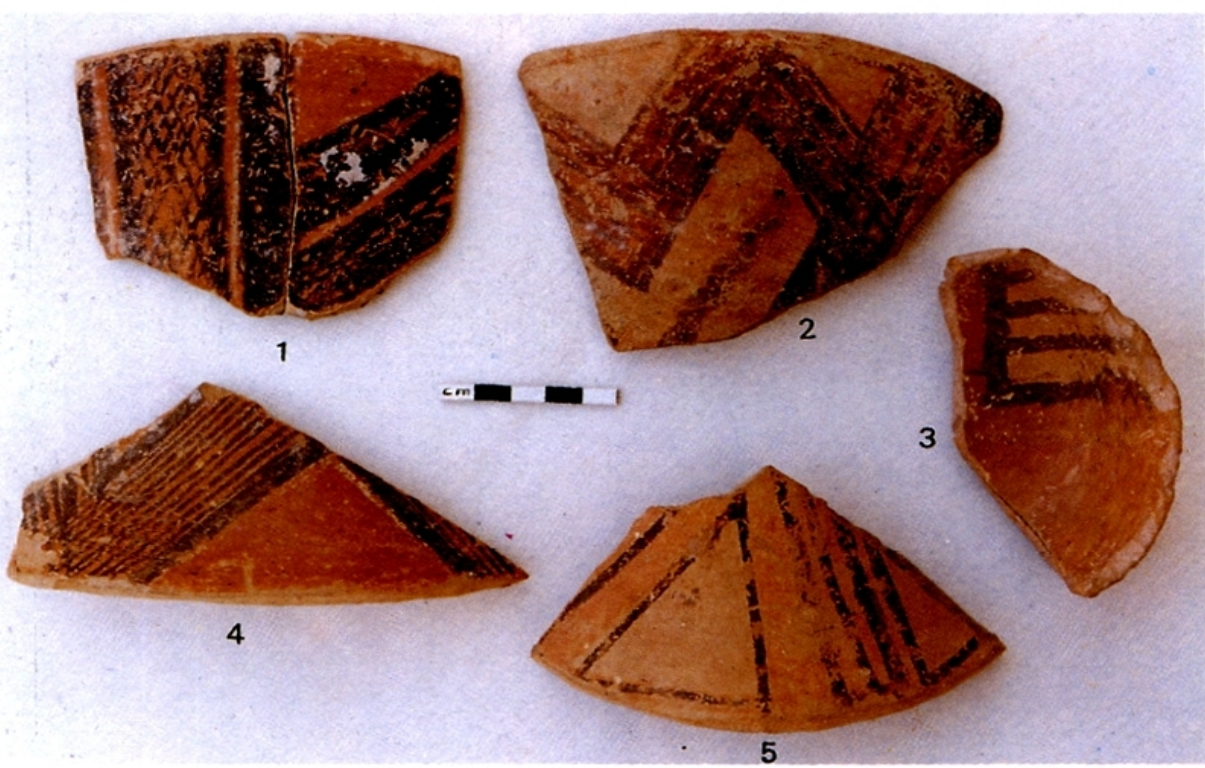

Fig. 23, no $1=$ fig. $11 \mathrm{D}$, no $1-\mathrm{n}^{\circ} 2=$ fig. $11 \mathrm{~B}, \mathrm{n}^{\circ} 3-\mathrm{n}^{\circ} 4=$ fig. $11 \mathrm{D}, 2-\mathrm{n}^{\circ} 5=$ fig. $11 \mathrm{~B}, \mathrm{n}^{\circ} 2$.

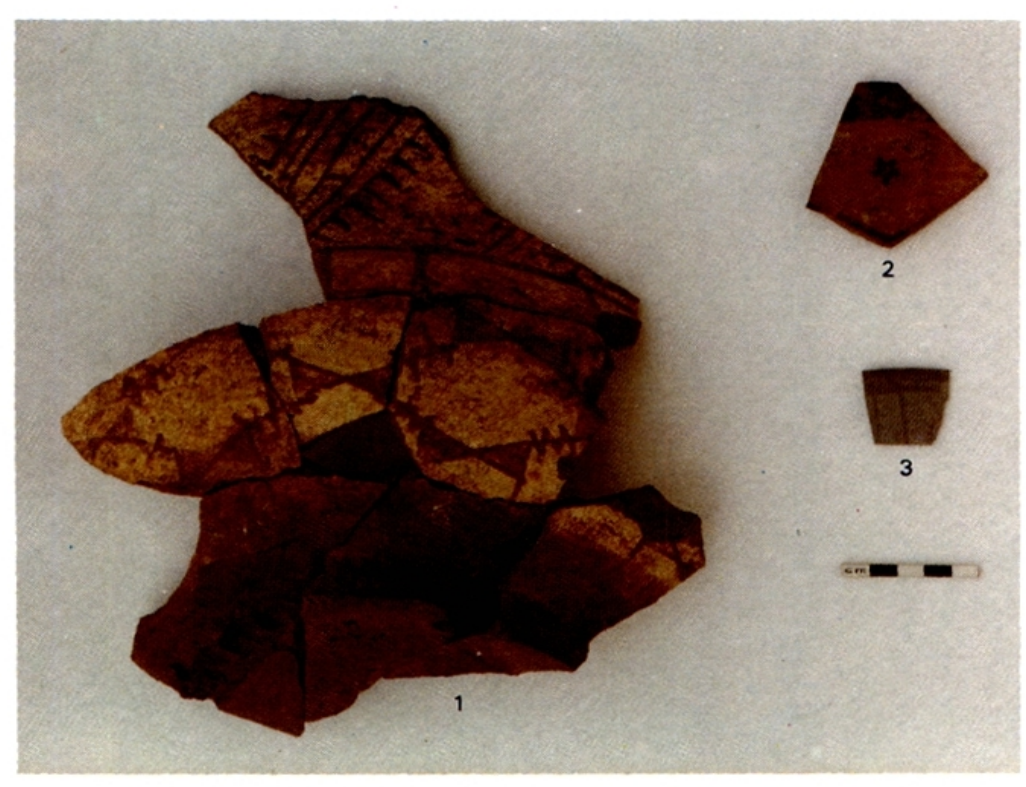

Fig. $25, n^{\circ} 1=$ fig. $10-n^{\circ} 3=$ fig. $8, n^{\circ} 13$. 
actuellement atteint $(-8,23 \mathrm{~m})$, elle n'est pas présente, tandis que la céramique à décor frotté y atteint $65 \%$; mais dès $-7,8 \mathrm{~m}$, la céramique à décor frotté ne représente plus que $40 \%$, tandis que cette céramique orange fait, avec d'autres catégories monochromes, son apparition. Elle cesse d'exister en même temps que la céramique à décor frotté, et il parait raisonnable de supposer qu'il s'agit là de récipients fabriqués de la même façon, mais non décorés. Toutefois elle ne varie pas de manière "gaussienne", et le décor ou non décor de ces récipients ne tiendrait donc qu'en partie à des facteurs chronologiques, mais aussi à des facteurs socio-fonctionnels. Elle est absente des niveaux récents de Plateia Magoula, et des sites de Makrychori 2 et d'Arapi.

c) une céramique rouge (Munsell $10 \mathrm{R} 5 / 4$ à $10 \mathrm{R} 6 / 6$ ). Sa facture est très proche de la précédente, et seule la couleur s'en distingue assez nettement. Elle varie curieusement de manière "gaussienne" inversée (tabl. 22); on pourrait croire alors, comme il est d'usage, avoir affaire à deux phénomènes distincts si, à défaut de techniques d'analyses plus complexes, son apparence ne semblait identique tout au long de la séquence, et si cette courbe bi-modale n'était pas celle de la céramique monochrome en général. Elle pourrait cependant représenter dans les niveaux anciens une variété de la catégorie précédente, et être plutôt liée dans les niveaux récents à l'essor de la céramique peinte en noir sur rouge, qui est de facture comparable. C'est après la céramique gris-surgris la seconde catégorie en importance parmi la céramique funéraire de Plateia Magoula Zarkou, et il n'est pas exclu que certains de ces vases aient pu avoir été peints, tant le sol y est peu propice à la conservation. Parmi les formes reconnaissables (fig. 17), on note des coupes curvilignes à
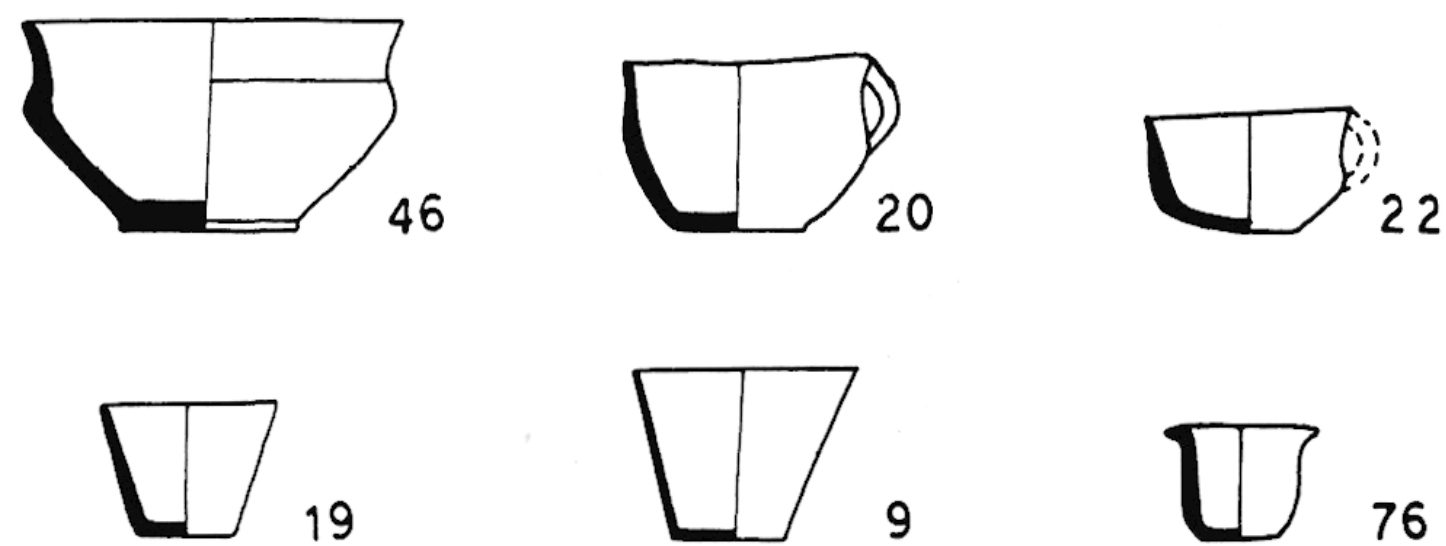

9

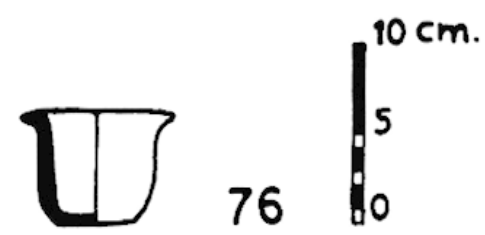

Fig. 17. - Formes de la céramique monochrome rouge (nécropole de Plateia Magoula Zarkou). Les chiffres sont ceux du catalogue de Gallis (1982), p. 119 sqq.

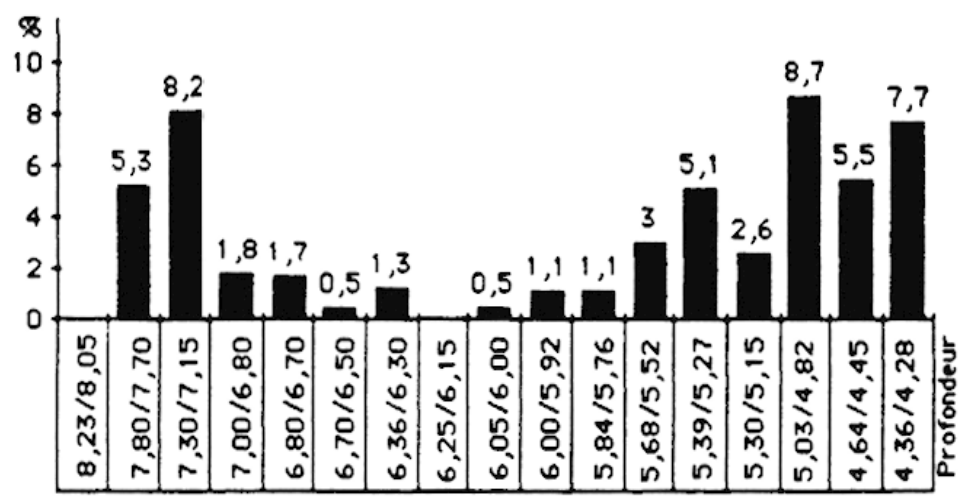

TABLEAU 22. - Evolution stratigraphique (en poids) de la céramique monochrome rouge fine et mi-fine sur le site de Plateia Magoula Zarkou. 
épaulement, proches de la céramique peinte en noir sur rouge ou de la céramique Larissa; des bouteilles à col cylindrique et anse(s) verticale(s); des gobelets tronconiques hauts, qui évoquent également la céramique peinte en gris-sur-gris. Cette catégorie est bien attestée à Makrychori 2 , où elle peut comporter exceptionnellement, quand elle est de très bonne facture, des décors lissés ou des décors peints en blanc comparables à ceux de la céramique Larissa. Elle évolue de fait, parallèlement à la céramique noire lissée à intérieur rouge (supra $\S \mathrm{a}$ ), vers une céramique monochrome rouge lissée, caractéristique des niveaux Arapi sur ce site et sur le site éponyme, et dont une partie est peinte en blanc ou en noir. Dans les périodes ultérieures, correspondant déjà à Dimini récent (périodes Aghia Sofia et Otzaki), cette céramique deviendra prépondérante, avec une ornementation très complexe.

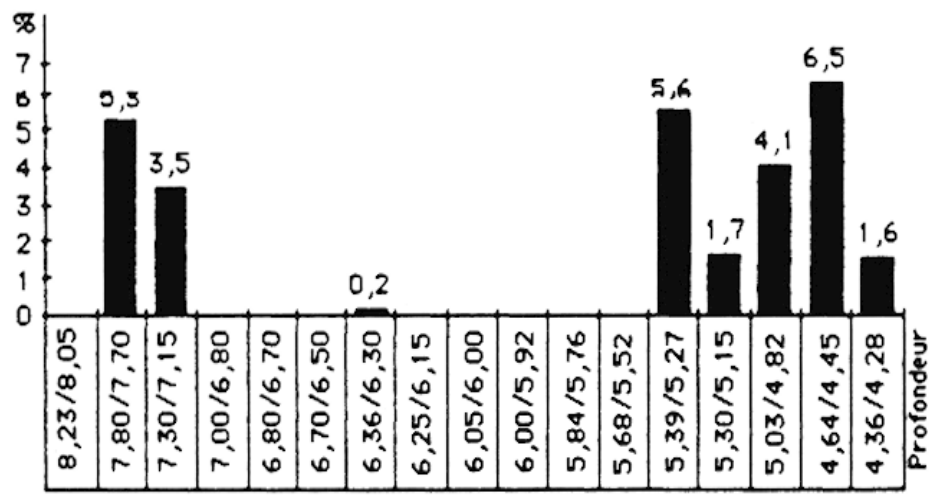

TABLEAU 23. - Evolution stratigraphique (en poids) de la céramique monochrome brun-clair fine et mi-fine sur le site de Plateia Magoula Zarkou.

d) une céramique brun-clair (sensiblement Munsell 5 Y 6/4). Cette catégorie se distingue bien de la variante "cuir" de la céramique Larissa (supra $\S \alpha$ ) par son traitement de surface inférieur et sa couleur. Comme la précédente, elle témoigne d'un développement bi-modal (tabl. 23); elle est complètement absente des niveaux moyens de Plateia Magoula Zarkou. Son développement dans les niveaux récents peut être mis en parallèle avec l'essor d'une céramique peinte brun-sur-brun. Cette catégorie est bien attestée à Makrychori 2.

e) une céramique brun-noirâtre (Munsell 5 YR 5/2 à 10 YR 6/2). Bien que cette catégorie varie parallèlement à la céramique monochrome en général, elle n'est peut-être pas entièrement homogène. La couleur brun-noirâtre peut en effet tenir à des "coups de feu " accidentels dus à une cuisson imparfaite, et il pourrait s'agir de fragments appartenant à des catégories différentes - un fait que l'analyse de fragments ne permet pas d'éviter, et qui ne modifie guère l'image statistique globale (tabl. 24).

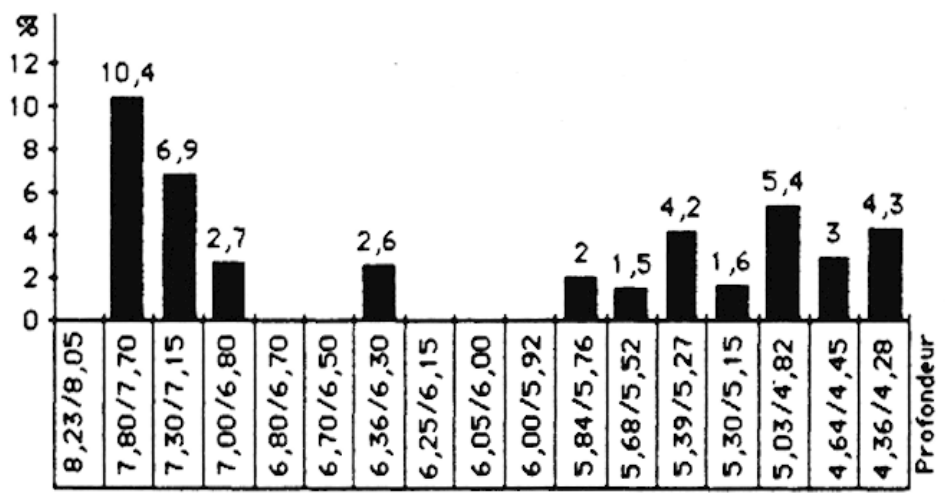

TABlEAu 24. - Évolution stratigraphique (en poids) de la céramique monochrome brun-noiråtre fine et mifine sur le site de Plateia Magoula Zarkou. 
f) une céramique beige (teinte entre Munsell 5 YR 8/4 et 10 YR 7/4). Cette catégorie est exclusivement présente dans les niveaux les plus récents de Plateia Magoula Zarkou, où elle augmente régulièrement, mais sans dépasser $3 \%$ (tabl. 25). On peut la mettre en parallèle avec l'essor de la céramique monochrome brun-clair et de la céramique peinte brun-sur-brun. Il est difficile de discerner les formes sur cet échantillon réduit.

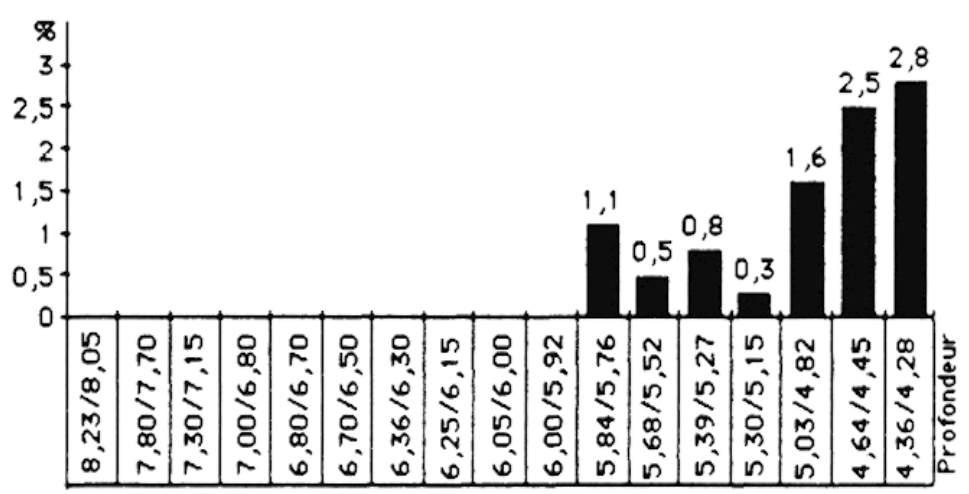

TABLEAU 25. - Evolution stratigraphique (en poids) de la céramique monochrome beige fine et mi-fine sur le site de Plateia Magoula Zarkou.

\subsection{La céramique grossière.}

Cette catégorie, en général peu ou pas analysée, représente jusqu'à $90 \%$ du poids total de la céramique recueillie dans les niveaux étudiés - ce qui signifie toutefois, vu la taille et le poids de ces vases, un nombre de récipients légèrement inférieur. Elle se distingue sans conteste du reste de la céramique par des parois plus épaisses, d'un cm au moins, un dégraissant grossier, un traitement de surface sommaire. La pâte est normalement de couleur claire, le cœur restant souvent noirâtre. A Plateia Magoula Zarkou, les formes sont essentiellement cylindriques ou faiblement ouvertes, et évoquent des vases de stockage ou de préparation alimentaire (fig. 14). Les formes plus profilées paraissent concerner des phases récentes et des vases de taille moyenne, comme c'est le cas à Arapi (Hauptmann et Milojčıć [1969], p. 35-36), à un moment où la céramique grossière représente 70 à $90 \%$ de la céramique. La seule autre évolution chronologique perceptible dans les formes concerne les fonds : ils comportent encore dans les niveaux les plus anciens une base marquée et légèrement creuse, dans la tradition des récipients Proto-Sesklo - et du Néolithique ancien balkanique en général; dans les niveaux moyens la base reste marquée, mais devient pleine, pour s'effacer progressivement en ne laissant plus subsister qu'un simple fond plat. Il n'y a normalement aucun moyen de préhension; ce n'est qu'à Makrychori et Arapi que tenons et anses sont présents, tout comme dans la période ultérieure de Dimini-Arapi.

La céramique grossière n'est qu'exceptionnellement décorée. On peut mentionner seulement à Plateia Magoula Zarkou sur quelques tessons l'existence d'un engobe blanc avec de rares traces de peinture brune (supra \$3.3.1.4.); et à Arapi la présence de quelques cordons digités (Hauptmann et Milojč́ćc [1969], pl. 3, 15-16).

Une autre évolution chronologique, intéressante, est celle de la proportion de céramique grossière dans son ensemble. Celle-ci suit en effet une courbe "gaussienne» durant toute la séquence de Plateia Magoula Zarkou. De $30 \%$ du poids total dans les 
niveaux fouillés les plus anciens, cette proportion s'accroît régulièrement pour atteindre jusqu'à $85 \%$ dans les niveaux intermédiaires, puis redécroître progressivement pour se stabiliser autour de $60 \%$ dans les niveaux supérieurs (tabl. 26). A Makrychori 2, où cette proportion est de toute façon plus forte, elle décroît néanmoins de 90 à $80 \%$ durant le mètre et demi de stratigraphie de la période Tsangli-Larissa (tabl. 9). A Arapi, dans les niveaux correspondants, elle est d'au moins $70 \%$ (tabl. 18; Hauptmann et Milojč́ćc [1969], p. 96 et 104), mais elle décroîtra fortement durant la période Arapi, où elle ne dépasse plus $45 \%$. Ainsi l'on assisterait durant les niveaux moyens de Plateia Magoula Zarkou à un effacement des catégories décorées ou monochromes demi-fines, au profit de récipients de facture plus grossière. Et lorsque la céramique grossière tendrait à nouveau à décroître, elle se stabiliserait néanmoins dans des proportions encore nettement supérieures à ce qu'elles étaient au départ, pour ne retrouver des proportions basses qu'ultérieurement, dans la période Arapi, qui est aussi un moment d'inventivité et de développement de la qualité de la céramique et de son ornementation.

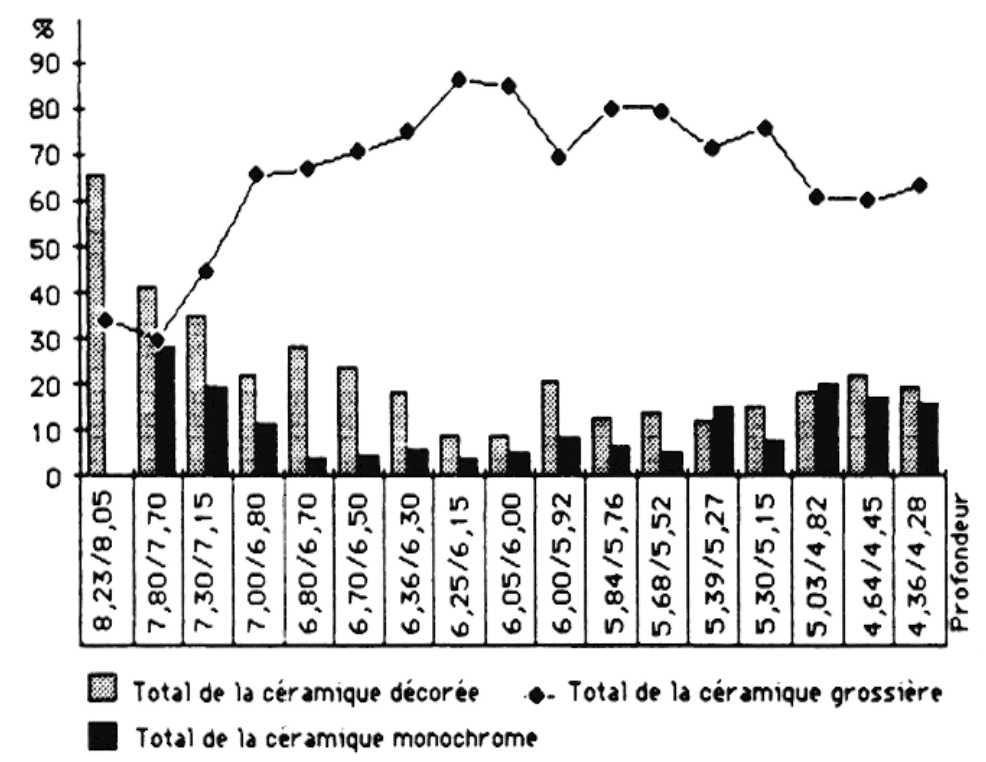

Tabieau 26. -- Evolution stratigraphique comparée à Plateia Magoula Zarkou de la céramique décorée, de la céramique monochrome fine et mi-fine et de la céramique grossière.

\section{INTERPRETATION ET HYPOTHESES DE RECHERCHE.}

\subsection{La chronologie.}

La durée approximative de la séquence Tsangli-Arapi, c'est-à-dire de la culture Dimini ancien, est sans doute, d'après les données du radiocarbone non calibrées (Theocharis [1981], p. 166; Treuil [1983], p. 117 sq; Kotsakis [1983], p. 36 et 49), d'environ cinq siècles, comme pour l'ensemble du "Néolithique moyen" balkanique, avec une origine vers 4.500-4.200 ans avant notre ère et une fin vers 3.800 ans. Il s'agit donc d'une longue séquence. De fait, les couches Tsangli attestées sur les différents sites thessaliens ont une épaisseur moyenne de 1,5 à $2 \mathrm{~m}$. Il paraît donc raisonnable d'envisager une subdivision de cette période, compte-tenu des nombreuses évolutions 
stratigraphiques que nous venons de voir, et dont l'interprétation ne peut être pour l'essentiel que chronologique. Les différents diagrammes proposés ici (tabl. 27-28) permettent de préciser ces évolutions. On pourrait ainsi distinguer :

1) une période Sesklo récent : une définition plus exacte de cette période nécessiterait une périodisation statistique précise de Sesklo, pour laquelle les travaux les plus récents (Motrier [1981], Kotsakis [1983]) ne fournissent encore que des suggestions. On y trouverait notamment une tendance à la simplification du décor peint en brun-rouge sur engobe blanc, tandis que la céramique à décor frotté connaitrait son apogée en quantité. En l'absence de données d'ensemble, il est difficile de raccorder les niveaux inférieurs de Plateia Magoula Zarkou à des points précis d'autres séquences stratigraphiques, même si l'on peut estimer que les niveaux compris entre $8,25 \mathrm{~m}$ (le point le plus bas actuellement atteint) et environ $6,5 \mathrm{~m}$, doivent se placer dans une telle période. La très grande rareté de la céramique peinte en brun-rouge sur blanc dans les niveaux les plus profonds pourrait également, vu l'étroitesse du sondage, tenir à des différences fonctionnelles de distribution. On noterait néanmoins une réduction progressive de la céramique décorée, associée au développement d'une céramique fine non décorée, puis à l'augmentation en proportion de la céramique grossière.

2) une période de transition dile "de Zarko" : cette période originale, révélée par le sondage de Plateia Magoula Zarkou, se placerait sur ce site entre 6,5 et $5 \mathrm{~m}$ environ. Elle se caractérise notamment par la survivance, sous une forme "dégénérée", des techniques de décor du Sesklo récent (décor frotté, décor peint en brun-rouge) et par l'apparition foisonnante de techniques nouvelles, dont certaines ne dépasseront pas cette période, et qui restent faiblement standardisées (peintures rouges diverses, polychromies diverses), tandis que d'autres types nouveaux tendent à se stabiliser progressivement (céramique gris-sur-gris, céramique noire lissée Larissa). La plupart de ces phénomènes nouveaux peuvent être dérivés, avec des perfectionnements ou des innovations particulières, des expérimentations aussi, de techniques plus anciennes. Il en va de même pour les formes et pour les décors : le style Sesklo, à chevrons enchainés continus, ou les motifs du décor frotté, qui lui sont proches, en arêtes de poisson couvrantes, se poursuivent. La céramique grossière augmente dans des proportions considérables, au détriment de la céramique décorée comme de la céramique monochrome mi-fine. Les diagrammes 27 et 28 , qui regroupent quantitativement et stratigraphiquement en trois ensembles les différents types céramiques des trois premières périodes considérées ici, permettent de vérifier pour Plateia Magoula Zarkou la consistance de la périodisation proposée. Outre la stratigraphie de Plateia Magoula Zarkou, les fouilles anciennes de Tsani et Tsangli, où la céramique gris-sur-gris précède nettement l'émergence de la période TsangliLarissa et où, ainsi qu'à Tsapocha, Nessonis, Otzaki et Servia, une céramique "proto-gris-sur-gris" paraît présente, tendent à confirmer l'existence d'une telle période de transition et d'expérimentation (Wace et Thompson [1912], p. 102 et 142, tabl. 63 et 90 ; Hauptmann et Milojčćć [1969], p. 47; Theocharis [1973] p. 79 et [1981] p. 122; Ridley et Wardle [1979], p. 213 et 217).

3) une période Tsangli-Larissa ancienne, ou "de Larissa": dans cette période, représentée notamment à Plateia Magoula Zarkou après $5 \mathrm{~m}$, mais aussi sans doute à Otzaki (HauptManN [1981]), l'ensemble des techniques appartenant encore à la tradition Sesklo a définitivement disparu. Néanmoins les techniques maintenant stabilisées propres à Tsangli-Larissa (gris-sur-gris, mat brun-sur-brun, noir sur rouge) s'inspirent encore largement de la tradition ornementale Sesklo du style continu (cf. aussi HauptMann et Milosčrć [1969], p. 37), même si la tendance vers un style plus segmenté se développe. Les écuelles de la céramique noire lissée Larissa, mais aussi de la céramique peinte ou de la céramique monochrome ont plutôt un profil curviligne, avec des épaulements plus ou moins marqués. 
4) une période Tsangli-Larissa récente, ou "de Tsangli : cette période est bien caractérisée à Makrychori 2 et à Arapi, entre autres. Si les différences avec Plateia Magoula Zarkou peuvent tenir en partie à une variabilité géographique (la concentration de la céramique noire lissée Larissa en Thessalie orientale), le fait marquant est le développement stylistique de la segmentation, tant dans les formes, avec des profils beaucoup plus carénés, que dans l'ornementation, avec la disparition des chevrons enchaînés continus au profit d'éléments verticaux isolés - aussi bien dans le décor peint qu'avec le développement du décor lissé. On note aussi des techniques nouvelles (certaines polychromies, l'engobe blanc crayeux, la céramique rouge lissée éventuellement à décor peint en blanc) apparaissent, qui deviendront prépondérantes dans la période Arapi.

5) la période Arapi : celle-ci, qui n'est pas étudiée ici, se caractérise donc, en continuité avec la période précédente, par une céramique monochrome polie noire ou rouge, une céramique peinte en blanc sur fond noir ou rouge, une céramique polychrome noire et rouge sur engobe blanc, tandis que la céramique grossière recule fortement. Au vu de l'épaisseur des couches, qui dépassent rarement un mètre sur les différents sites, elle est sans doute plus brève que l'ensemble des périodes Tsangli-Larissa, et n'est pas encore subdivisible. On note que certains éléments annoncent à leur tour, notamment à Aghia Sofia, les phases les plus anciennes de Dimini récent : apparition de thèmes curvilinéaires complexes et enchaînés, métopage de la surface à décorer, et plus généralement standardisation croissante et restriction en nombre des catégories céramiques, indices sans doute de l'émergence d'un artisanat plus spécialisé.

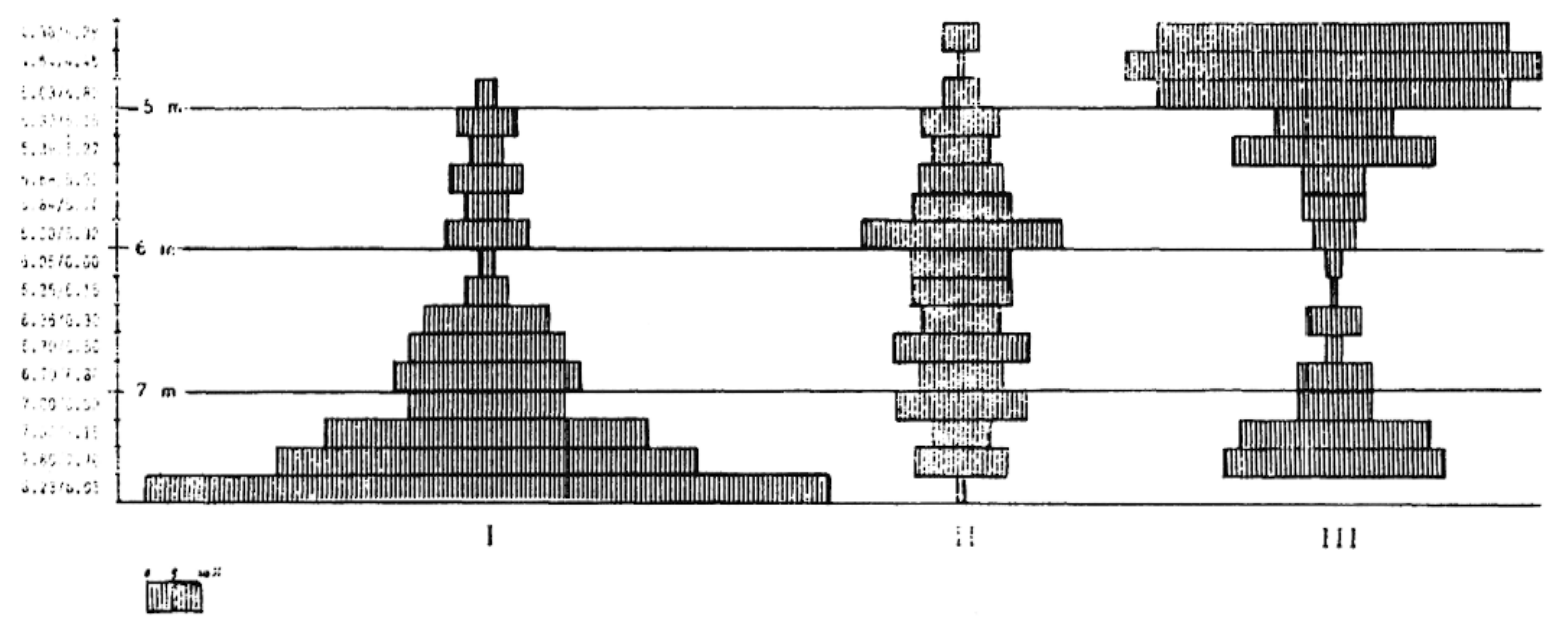

TABlEAu 27. - Évolution stratigraphique cumulée des trois principales associations céramiques de Plateia Magoula Zarkou. Groupe I : céramique à décor peint en brun-rouge "classique", céramique à décor frotté, céramique à décor imprimé; Groupc II : céramique à décor peint en brun-rouge sur clair "dégénéré ", céramique "proto-gris-sur-gris", céramique gris-noirâtre, céramique polychrome rouge à triangles noirs, céramique peinte en rouge, céramique monochrome orange; Groupe III : céramique gris-sur-gris, céramique "Larissa " céramique peinte noire sur rouge, cèramique peinte brun-sur-brun, céramique peinte sur engobe blanc, céramique monochrome brun-clair, céramique monochrome rouge, céramique monochrome brun-noirâtre, céramique monochrome beige. 


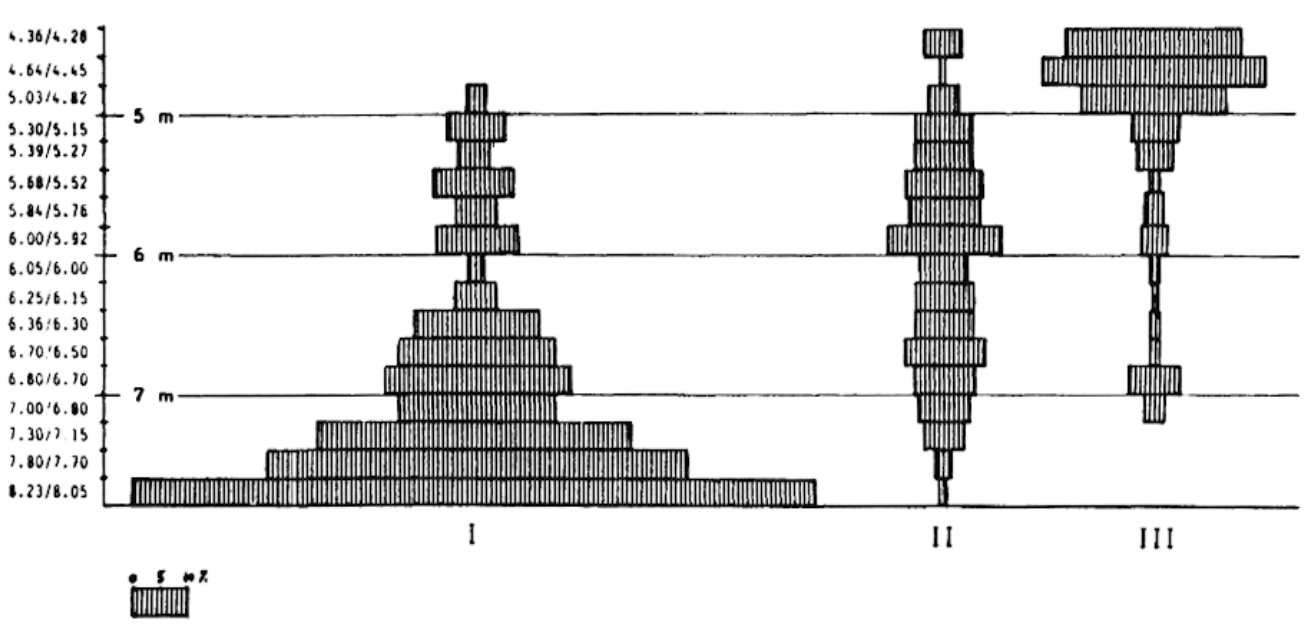

Tableau 28. - Evolution stratigraphique cumulée des trois principales associations céramiques de Plateia Magoula Zarkou, céramique monochrome fine et mi-fine exclue (pour la légende, cf. tabl. 27).

\subsection{Variabilité régionale et distribution spatiale.}

La forme la plus simple de variabilité spatiale est celle interne à chaque site. Elle ne peut pratiquement pas être estimée en Thessalie, où toutes les fouilles récentes se sont limitées à des sondages. Notons cependant que sur les fouilles, plus étendues, de D. Theocharis sur le site éponyme de Sesklo, les différences quantitatives entre structures d'une même phase peuvent être sensibles (Kotsakis [1983]). Au moins deux types de variabilité régionale peuvent sinon être reconnus : entre grandes régions, c'est-à-dire essentiellement entre l'Est et l'Ouest de la Thessalie, mais aussi entre sites d'une même région. La Thessalie occidentale est caractérisée notamment, on l'a vu, par la prédominance du gris-sur-gris, et par certains types de polychromie, notamment celles à dominante rouge. En Thessalie orientale domine en lieu et place la céramique Larissa noire lissée (cf. tabl. 9, 18). Cette donnée a parfois été citée à l'appui de l'hypothèse migrationniste, la Thessalie orientale étant la plus proche des régions à céramique noire lissée (Holmberg [1964] p. 347; Gallis [1987] p. 162). On a vu aussi que la céramique à décor frotté paraissait plus ancienne dans le Nord de la Thessalie que dans le Sud, mais que dans le Nord, il existait aussi une variabilité sensible entre Plateia Magoula Zarkou et Otzaki ; de même, le décor peint mat noir sur rouge est quantitativement plus important à Plateia Magoula Zarkou qu'à Makrychori 2, proportion inversée sur ce dernier site. A l'intérieur de la Thessalie nord-orientale, on remarque d'autres différences entre sites proches. Ainsi Makrychori connait dès la phase Tsangli la technique "black topped" et la céramique rouge fine, qui n'apparaissent semble-t-il à Arapi qu'avec la période Arapi. Sans préjudice d'interférences chronologiques, certains sites pourraient donc avoir un développement stylistique plus novateur que d'autres. C'est dans cette perspective qu'un examen détaillé des données, maintenant nombreuses, des prospections de surface en Thessalie, pourrait apporter des résultats très prometteurs, notamment quant aux interactions entre sites.

Une première approche de l'implantation des sites montre en effet, au moins pour la Thessalie orientale, une image intéressante (fig. 1). Si l'on considère les quelque 140 sites occupés pendant l'une ou l'autre des périodes Sesklo et Tsangli, ou pendant les deux successivement, on constate que le nombre de sites est moitié moindre durant la période 
Tsangli. Ainsi, $75 \%$ des sites occupés pendant la période Sesklo ne sont plus occupés durant la période Tsangli. Symétriquement, parmi les sites de la période Tsangli, la moitié occupe des emplacements identiques à ceux des villages Sesklo, tandis que l'autre moitié fait l'objet d'implantations nouvelles. Ces chiffres n'impliquent pas nécessairement une réduction de l'implantation, car d'une part tous les villages Sesklo ne sont pas forcément contemporains et cette culture est de toute façon plus longue en durée que la période Tsangli; d'autre part la taille des villages n'est pas nécessairement la même. A l'échelle de toute la Thessalie, ces nouvelles implantations Tsangli représentent environ le dixième des sites néolithiques thessaliens connus (Gallis [1987] p. 162). La distribution globale est aussi différente, les implantations Sesklo formant des groupements en certains points, notamment à proximité des cours d'eau, tandis que les implantations TsangliLarissa sont beaucoup plus régulièrement espacées (fig. 1). Il semble donc que la période Tsangli coincide avec un important remaniement de l'habitat humain comme d'ailleurs dans l'ensemble du "Néolithique moyen" balkanique. Cela n'apporte néanmoins aucun argument décisif à l'appui d'une interprétation historique particulière. Ainsi l'existence vers la fin de la séquence Sesklo de couches d'incendie, régulièrement citée, peut tenir à de simples accidents, dont la taille réduite des sondages peut d'ailleurs masquer le caractère ponctuel; en outre, certains de ces incendies, comme à Servia, ne coincident pas avec la dernière occupation Sesklo. De même on constate à Plateia Magoula, Makrychori 2 et Arapi, c'est-à-dire précisément sur les rares sites à avoir fait l'objet de fouilles stratigraphiques récentes, que l'abandon ou la fondation d'un village ne coïncide pas avec la grande coupure culturelle Sesklo/Dimini, mais se situe à l'intérieur même de la période Tsangli-Larissa. L'apparition de nécropoles à incinération, rite inconnu depuis Proto-Sesklo, à Soufli Magoula et à Plateia Magoula Zarkou est un autre indice de transformations culturelles dont l'examen exhaustif ne devrait évidemment pas se limiter à l'analyse des catégories céramiques.

\subsection{Phénomènes fonctionnels, technologiques et stylistiques.}

\subsubsection{INTERPRÉTATION FONCTIONNELLE ET SOCIALE DES CATÉGORIES CÉRAMIQUES.}

La comparaison entre formes et catégories (tabl. 11) montre que l'ensemble de la céramique fine et mi-fine, ornée ou non, semble occuper une position fonctionnelle identique. Les principales formes : bouteilles, écuelles ouvertes, gobelets hauts, sont attestées dans toutes les catégories, avec une morphologie voisine. Seule la céramique Sesklo classique à peinture brun-rouge sur engobe blanc possède un répertoire de formes plus restreint; c'est aussi une céramique très minoritaire, et son éventail fonctionnel pourrait être plus réduit. La céramique grossière s'isole nettement de l'ensemble, avec des formes spécifiques. On aurait bien deux ensembles, le premier correspondant à des usages sociaux, réclamant une céramique de qualité, l'autre à des usages plus immédiatement utilitaires. Il parait en revanche malaisé de discerner des différences fonctionnelles à l'intérieur de la céramique non-grossière.

La répartition de la céramique funéraire dans la nécropole à incinération de Plateia Magoula Zarkou (Gallis [1982]) va dans le mème sens. Céramique gris-sur-gris, céramique noire lissée et céramique monochrome rouge-orange sont indifféremment associées (tabl. 29), avec des formes spécifiques mais voisines, la fréquence des associations dans une même tombe entre récipients de catégories différentes ne semblant dépendre que du nombre de récipients lui-même. Leurs proportions ne reflètent 


\begin{tabular}{|c|c|c|c|c|c|}
\hline & & & & & Divers \\
\hline 1 & 0 & & & & \\
\hline 2 & & & & $R$ & \\
\hline 4 & 0,0 & & & & \\
\hline 5 & $G$ & $G$ & & & \\
\hline 6 & & & & $G$ & \\
\hline 7 & & & & & \begin{tabular}{|l} 
Vase globulaire gris \\
\end{tabular} \\
\hline 10 & $\theta$ & & & & \\
\hline 12 & G & & & & \\
\hline 13 & & & $R$ & & \\
\hline 14 & $G$ & $R$ & & $R$ & \\
\hline 15 & 0 & & & & \\
\hline 16 & & & & $R$ & \\
\hline 18 & & & & 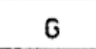 & \\
\hline 21 & & & & $G$ & \\
\hline 22 & & $R$ & & $N$ & \\
\hline 24 & & $R, G$ & & 0,0 & Vase zoomorphe noir \\
\hline 26 & 0 & 0 & & & \\
\hline 27 & & & & 0 & \\
\hline 28 & & & $\mathrm{~N}$ & $\mathrm{G}, \mathrm{N}$ & \\
\hline 32 & & & & $G$ & \\
\hline 33 & $G$ & $G$ & & . & Micro-bouteille biconique rouge \\
\hline 34 & & $R$ & & $R$ & \\
\hline 35 & & & & G & \\
\hline 36 & & $G$ & & $G$ & \\
\hline 37 & & & & G & \\
\hline 38 & & & & G & \\
\hline 39 & $G$ & & G & & \\
\hline 40 & 0 & & & & \\
\hline
\end{tabular}

Tableau 29. - Occurrences des différentes catégories de récipients déposés dans les tombes de la nécropole à incinération de Plateia Magoula Zarkou (d'après Gallus [1982]). G : céramique gris-sur-gris; $\mathrm{N}$ : céramique noire lissée; $\mathbf{R}$ : céramique monochrome rouge.

cependant pas celles de la céramique d'habitat, même en en excluant la céramique grossière (tabl. 10). La céramique gris-sur-gris est sur-représentée, avec $60 \%$ des récipients déposés, alors qu'elle ne dépasse pas en poids $7 \%$ du total de la céramique d'habitat, ou $19 \%$ du total de la céramique non-grossière, tandis que la céramique monochrome est sous-représentée, la céramique noire lissée occupant une position moyenne. Cette répartition semble donc valoriser les productions selon leur degré de difficulté technique. L'interprétation est limitée par le rite de l'incinération, qui n'a pas permis d'identifier les âges et les sexes. On note cependant (tabl. 29) que le dépôt funéraire comprend au moins un vase de grande taille, associé ou non à d'autres récipients; et que les deux principales catégories de vases, les bouteilles et les vases 
ouverts à parois hautes, s'excluent normalement à l'intérieur d'une même tombe et sont en quantité sensiblement égale, sans former non plus de groupements particuliers à l'intérieur de la nécropole. Il serait tentant de donner à ce fait une signification sexuelle, que des analyses anthropologiques limitées pourraient tester. Dans tous les cas, l'opposition semble plus concerner les différences morphologiques et fonctionnelles des récipients que leurs différences technologiques. Ces différences sont évidemment recoupées par la variabilité géographique, puisque les tombes à incinération de la nécropole de Soufli Magoula, situee en Thessalie orientale, contiennent essentiellement de la céramique Larissa noire lissée. L'identité de fonction entre catćgories est également illustrée par les formes et les motifs communs qu'elles partagent indifféremment (cf. tabl. 11 ; comparer fig. $8 \mathrm{n}^{\circ} 13 ; 25, \mathrm{n}^{\text {os }} 2-3$ ).

\subsection{2. Évolutions Chronologiques des Catégories cÉramiques.}

On a pu voir qu'il y avait dans le temps entre catégories des glissements techniques progressifs. Ainsi, la céramique peinte en brun-rouge sur blanc évolue vers des formes de plus en plus "dégénérées", puis vers de simples décors linéaires peints en rouge, qui n'auront eux-mêmes pas de successeurs. La céramique à décor frotté est l'une des origines techniques de la céramique gris-sur-gris, par l'intermédiaire de deux catégories de transition ; cette céramique influe aussi sur le lissage de la céramique polychrome rouge à triangles noirs. Du point de vue du style ornemental, les motifs de chevrons continus de la céramique peinte en brun-rouge se retrouvent tant dans la céramique gris-sur-gris que dans les céramiques à peinture mate noire ou brune. Il y a donc continuité, mais aussi, pendant la période intermédiaire dite "de Zarko», "déstandardisation" typologique. Ainsi, une partie des innovations réelles constatées (peinture noire au manganèse, céramique noire lissée, céramique grise, polychromie) n'apparaissent pas de manière abrupte, mais plutôt progressive, voir tâtonnante. Seule la céramique noire lissée paraît une invention relativement rapide mais, nous l'avons vu, nous ne disposons pas de bonnes stratigraphies Sesklo/Dimini en Thessalie orientale.

A ces phénomènes stylistiques se superpose une autre tendance, celle qui pendant cette même période "de Zarko" fait diminuer la proportion de céramique décorée parmi la céramique fine ou mi-fine, et fait augmenter la quantité de céramique grossière. Il y a donc un glissement entre ces trois catégories du décoré vers le non décoré, et du non décoré vers le grossier, accompagné de techniques d'exécution du décor moins élaborées (céramique peinte en brun-rouge dite "dégénérée», peintures rouges diverses). Ainsi la tendance d̀ une production moins soignée coïncide avec cette déstandardisation stylistique. Dans les périodes ultérieures Tsangli-Larissa, et plus encore Arapi, le retour vers des types stables coincide avec une nette diminution de la céramique grossière.

Ainsi les proportions différentes entre catégories céramiques obéissent-elles à des facteurs divers : chronologiques, avec cette tendance stylistique à l'augmentation, puis à la diminution des céramiques moins soignées; géographiques, avec les différences symétriques Ouest/Est dans la répartition de la céramique noire lissée et de la céramique gris-sur-gris; socio-fonctionnels, avec, dans la nécropole de Plateia Magoula, les associations et exclusions entre catégories de récipients, ou la valorisation de certaines catégories céramiques. 


\subsection{Comparaisons inter-régionales.}

La zone proprement thessalienne semble être limitée au Sud par la vallée du Spercheios, et au Nord par celle de l'Aliakmon; à l'Ouest, la chaîne du Pinde est une limite probable, mais le Néolithique de l'Épire est presqu'inconnu. Dès la Béotie en effet, dans l'horizon Sesklo comme dans l'horizon Tsangli-Larissa, la Grèce centrale forme une région distincte, malgré les affinités stylistiques qui permettent les synchronismes. La faiblesse de la recherche n'a d'ailleurs pas permis encore la définition précise de groupes régionaux. La Grèce centrale permet d'ailleurs, par d'autres affinités stylistiques, de synchroniser avec Sesklo le "Néolithique moyen" du Péloponèse, caractérisé par la céramique "Urfirnis». L'horizon synchrone avec Tsangli-Larissa en Grèce centrale et méridionale a été caractérisé depuis longtemps (Goldman [1931], WALKer-Kosmopoulos [1948], Holmberg [1964], Schachermeyr [1976], Hauptmann et Milojčı́c [1969], HAUPTMANn [1981]). En revanche les horizons correspondant à la période Arapi et aux périodes Dimini récent sont mal attestés dans ces régions.

Vers le Nord, après la vallée de l'Aliakmon qui, avec Servia, appartient encore à la zone thessalienne malgré des liens déjà plus septentrionaux (RıdLEY et WARDLE [1979]), la coexistence d'une céramique sombre polie et de styles peints est caractéristique aussi bien de la Macédoine centrale, comme à Vassilika (Grammenos [1984]), que de la Macédoine orientale, comme à Akropotamos, Sitagroi I-II, Dikili Tach et du Sud-Ouest de la Bulgarie, comme à Bălgărčevo et Topolnica (Pellerin [1986]). La situation de la Macédoine occidentale, qui dans les périodes Dimini récent se situe à l'intérieur d'un continuum joignant la Thessalie à l'Albanie du Sud-Est, n'est pas connue.

\section{CONCLUSIONS ET DIRECTIONS DE RECHERCHE.}

Dans la perspective des études ultérieures dont le présent travail constitue la première étape, on soulignera en conclusion les points suivants :

a) L'apparition de la culture de Dimini se caractérise par un foisonnement de techniques nouvelles, dont l'essentiel se situe néanmoins en continuité avec les techniques antérieures.

b) L'ensemble de ces techniques donne une impression contradictoire de recherche, d'inventivité, mais aussi de "déstandardisation" typologique par rapport aux phases précédentes et suivantes. Il s'agirait de toute façon d'un moment historique très particulier.

c) Seule la céramique noire polie, par ailleurs connue à la même époque dans une grande partie de l'Anatolie et des Balkans semble un élément étranger, d'ailleurs plutôt confiné au Nord-Est de la région. La Thessalie en général et les stratigraphies analysées ici en particulier offrent donc une possibilité privilégiée d'étudier, en un moment d'importantes transformations, la part respective des traditions antérieures, des innovations locales et des apports externes.

d) Les recherches ultérieures devraient s'orienter à la fois vers l'analyse plus détaillée encore des stratigraphies de référence, ainsi que de celles qui sont encore presque 
inédites, vers la recherche de nouvelles séquences susceptibles de compléter les anciennes, et vers une mise en œuvre des données de prospection de surface, afin de préciser la nature des interactions locales entre communautés.

e) La partie suivante de cette étude sera consacrée à l'interprétation des analyses technologiques et physico-chimiques.

Jean-Paul Demoule, Kostas Galdis et Laurence Manolakakis.

\section{BIBLIOGRAPHIE}

H. Biesantz (1959) : "Die Ausgrabungen bei der Soufli-Magoula», AA (1959), p. 56-74.

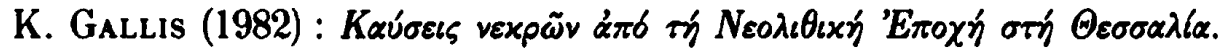

K. Gallis (1987) : "Die stratigraphische Einordnung der Larisa-Kultur : eine Richtigstellung", Prähistorische Zeitschrift 62, p. 147-163.

H. Goldman (1931): Excavalions at Euresis in Boeotia.

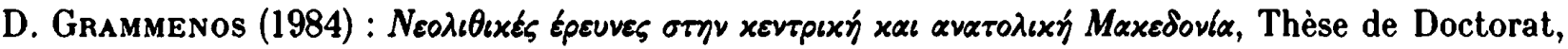
Université de Salonique.

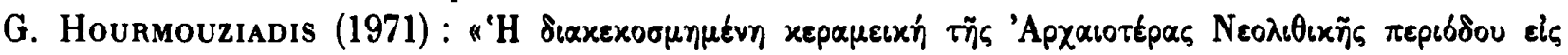

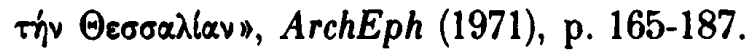

H. Hauptmann (1981): Die deutschen Ausgrabungen auf der Otzaki-Magula in Thessalien III. Das späte Neolithikum und das Chalkolithikum.

H. Hauptmann (1986) : "Probleme des Chalkolithikums in Griechenland", A beri balogh àdàm muzeum évkönyve VIII, Szekzard, p. 19-30.

H. Hauptmann et V. Milojčıć (1969): Die Funde der frühen Dimini-Zeil aus der ArapiMagula, Thessalien.

E. Holmberg (1964) : "The Appearance of Neolithic Black Burnished Ware in Mainland Greece», $A J A 68$ (1964), p. 343-348.

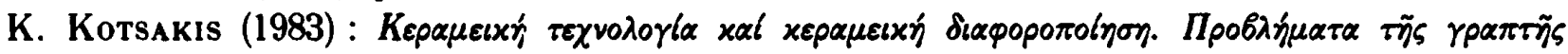

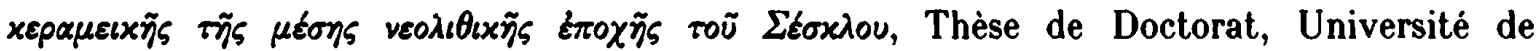
Thessalonique.

J. Letsch et W. Noll (1983) : "Mineralogie und Technik der frühen Keramiken Thessaliens", Neues Jahrbuch Miner. Abh. 147, 2, p. 109-146.

J. Lichardus, M. Lichardus-Itten, G. Bailloud et J. Cauvin (1985) : La Protohistoire de l'Europe. Le néolithique et le chalcolithique.

V. Mılosčıć (1949) : Chronologie der jüngeren Steinzeit Mittel- und Südost Europas.

V. MıLoJčıć (1959) : "Hauptergebnisse der deutschen Ausgrabungen in Thessalien (1953-1958)", Jahrbuch Röm.-Germ. Zentr. Mus. Mainz 6, p. 1-56.

V. Milojčıć, A. von den Driesch, K. Enderle et J. Milojčıćc-von Zumbusch (1976) : Die deutschen Ausgrabungen auf Magulen in Thessalien. 1966.

V. Milojčıć et J. Milojčıć-von Zumbusch (1983): Die deutschen Ausgrabungen auf der Otzaki-Magula in Thessalien, II-III. 
Y. MotTIER (1981) : Die deutschen Ausgrabungen auf der Otzaki-Magula in Thessalien II. Das mittlere Neolithikum.

G. Mylonas (1941) : "The site of Akropotamos and the Neolithic Period of Macedonia", AJA 45 (1941), p. 558-583.

C. Pellerin (1986) : Le Néolithique Moyen en Grèce du Nord, Thèse de Doctorat, Université de Paris I, 3 volumes.

C. Ridley et K.A. WARdle (1979) : "Rescue excavations at Servia 1971-1973. A preliminary Report", BSA 74, p. 185-230.

F. Schachermeyr (1976): Die Agäische Frühzeit I. Die vormykenischen Perioden des griechischen Festlandes und der Kykladen.

D. Theocharis (éd. 1973) : Neolithic Greece.

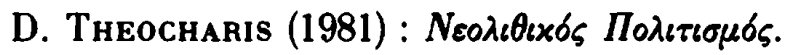

V. Trtov (1969) : Neolit. Grecii. Periodizacija i Chronologija, Moscou.

R. Treuil (1983) : Le Néolithique et le Bronze Ancien égéens.

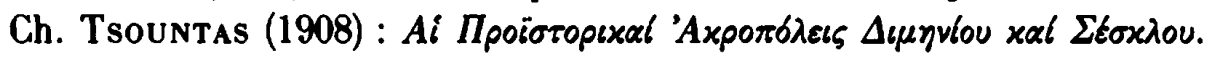

A.J.B. WAce et M.S. Thompson (1912) : Prehistoric Thessaly.

L. Walker-Kosmopoulos (1948) : The Prehistoric Inhabitation of Corinth.

M. WiJnen (1982) : The Early Neolithic I Seltlement at Sesklo : An Early Farming Community in Thessaly, Greece. 